\title{
Theoretical X-Ray Spectroscopy of Transition Metal Compounds
}

\author{
Sergey I. Bokarev*, Oliver Kühn ${ }^{\dagger}$
}

\section{Article Type:}

Advanced Review

\begin{abstract}
$\mathrm{X}$-ray spectroscopy is one of the most powerful tools to access structure and properties of matter in different states of aggregation as it allows to trace atomic and molecular energy levels in course of various physical and chemical processes. X-ray spectroscopic techniques probe the local electronic structure of a particular atom in its environment, in contrast to UV/Vis spectroscopy, where transitions generally occur between delocalized molecular orbitals. Complementary information is provided by using a combination of different absorption, emission, scattering as well as photo- and autoionization X-ray methods. However, interpretation of the complex experimental spectra and verification of experimental hypotheses is a non-trivial task and powerful first principles theoretical approaches that allow for a systematic investigation of a broad class of systems are needed. Focussing on transition metal compounds, $L$-edge spectra are of particular relevance as they probe the frontier $d$-orbitals involved in metal-ligand bonding. Here, near-degeneracy effects in combination with spin-orbit coupling lead to a complicated multiplet energy level structure, which poses a serious challenge to quantum chemical methods.

Multi-Configurational Self-Consistent Field (MCSCF) theory has been shown to be capable of providing a rather detailed understanding of experimental X-ray spectroscopy. However, it cannot be considered as a 'blackbox' tool and its application requires not only a command of formal theoretical aspects, but also a broad knowledge of already existing applications. Both aspects are covered in this overview.
\end{abstract}

\footnotetext{
${ }^{*}$ Institut für Physik, Universität Rostock, Albert-Einstein-Str. 23-24, 18059 Rostock, Germany

${ }^{\dagger}$ Institut für Physik, Universität Rostock, Albert-Einstein-Str. 23-24, 18059 Rostock, Germany
} 


\section{INTRODUCTION}

Spectroscopy is one of the most powerful tools in physics and chemistry and, in particular, X-ray spectroscopy attracts special attention not at least due to the ongoing developments at X-ray Free Electron Laser (XFEL) facilities and High Harmonic Generation (HHG) sources 1]2 As a consequence of the localized nature of the core orbitals, the transition operator acts locally and that is why X-ray excitation probes the local electronic structure of a particular atom embedded in its chemical environment. This is in contrast to, e.g., UltraViolet/Visible photon energy range (UV/Vis) spectroscopy, where transitions usually occur between delocalized valence Molecular Orbitals (MOs). The combination of different absorption, emission, scattering, photoionization, and diffraction Xray techniques allows addressing various aspects of static properties as well as photoinduced and chemical dynamics in steady-state and time-resolved X-ray spectroscopy $1[3] 6$ Sharpening the experimental probe does not guarantee, however, an increase of the acquired knowledge as the complexity of the detected signal increases as well when addressing more and more intricate effects. Thus, for interpretation of the experimental data, theoretical modeling is mandatory.

This review presents an overview on theoretical approaches with a focus on first principles electronic structure methods and in particular on multi-reference wave function techniques. Applications to the interpretation of experimental data will be shown to provide a means for dissecting different structural and dynamical problems. We will restrict ourselves to isolated molecules and complexes as they appear in the gas or solution phases. Extended periodic systems like crystals are explicitly excluded from consideration as respective theoretical models represent a huge selfstanding body of methods which are reviewed elsewhere $[5,7,8$ Here, we address only discrete the pre-edge structure leaving XANES and EXAFS parts of the absorption edge aside since especially the latter provides more information on the geometric rather than electronic structure .9

Although computational X-ray methods have been developing apace with quantum chemistry since its infancy, the 21st century heralds an explosive growth of theoretical studies of X-ray spectra. These studies are mainly devoted to the $K$-edge $(1 s)$ spectra of second period and heavier elements using single reference methods. It is barely possible and not aimed in the current review to cover them all, including the numerous applications (for reviews see References $5,10-12$ ). Therefore, mostly the soft X-ray photon energy range (0.1-1.0 keV) and 4th period Transition Metal (TM) complexes will be considered. Thus, mainly metal $L$-edges will be discussed corresponding to excitation or ionization from $2 p$ core orbitals. 


\section{X-RAY SPECTROSCOPY}

\subsection{What makes it special?}

Spectroscopy in the UV/Vis spectral range is a standard tool for probing electronic transitions and related processes, taking place within the valence state manifold. Here, participating electronic states often exhibit rather delocalized electron densities. This makes an interpretation in terms of local changes difficult, e.g., with respect to specific bonds. In contrast, X-ray transitions involve at least one orbital, which is localized on an atom, thus providing a local probe of the electronic structure. Moreover, core level energies vary vastly between different atoms what provides element specificity. Further, core orbitals with non-zero angular momentum lead to pronounced relativistic effects, e.g., caused by Spin-Orbit Coupling (SOC). Conventional X-ray spectroscopy is plagued by the rather short lifetime of the core-hole (4-10 fs), which limits the resolution (a possible way to overcome this problem is High Energy Resolution Fluorescence Detection X-ray Absorption Spectrum (HERFD-XAS) ${ }^{13}$ ). However, this can be turned into an advantage by using the corehole lifetime to clock ultrafast dynamics ("core-hole clock")! 14 Finally, X-ray wavelengths are in the range of relevant molecular length scales, enabling better spatial and time resolution.

The list of problems, which X-ray spectroscopy typically addresses includes probing the chemical environment and bond distances, $\sqrt[9]{15}\left[17\right.$ the nature of chemical bonds $\frac{18}{20}$ as well as oxidation, spin, and solvation states $\sqrt[21]{24}$ Further, it is indispensable for the investigation of solids and surfaces $\sqrt[3 \mid 4]{25 \mid 26}$ While steady-state X-ray spectroscopy is well-established, emerging time-resolved experiments are pushing the limits to enter the regime of even the fastest nuclear and electron dynamics. This becomes possible due to the fact that the optical cycle of X-rays is in the few attosecond range. Among others, $\frac{1] 6}{6}$ recent examples include, e.g., the study of the (photo)reaction dynamics of acetylene isomerization upon core-hole formation,, 27 photodissociation of simple flourides, ${ }^{28}$ ligand exchange $\mathrm{e}^{22}$ and transient photodynamics 39 in metal complexes, photocatalytic processes on metal surfaces,, $32\left[33\right.$ nanosecond dynamics of dissociation and reassociation of insulin, $\frac{34}{34}$ and ultrafast electron and nuclear wave packet dynamics using time-resolved and transient photoelectron spectroscopy 35

The advantages of X-ray as compared to UV/Vis spectroscopy come at the price of requiring large scale facilities like synchrotrons and XFELs. However, recent progress in HHG sources scales down the size of the setups to the table-top. Moreover, prominent recent developments include low-power X-ray (bremsstrahlung) tubes $\$ 37$ and plasma-based X-ray pulse generation. 
anticipated that within a few years a table-top intense and stable source of isolated ultrafast XUV and X-ray pulses will appear what will herald the "golden age" of frequency- and time-resolved $\mathrm{X}$-ray spectroscopy giving rise to a plethora of new techniques and perspective directions. ${ }^{2}$

\section{$2.2 \quad$ Types of X-ray probes}

(a)

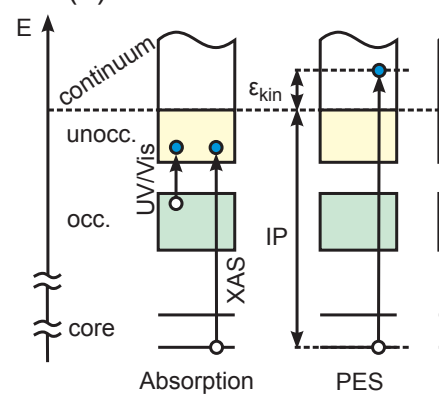

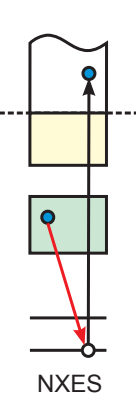

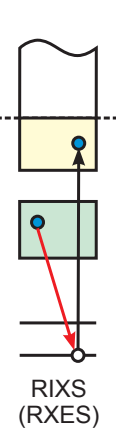

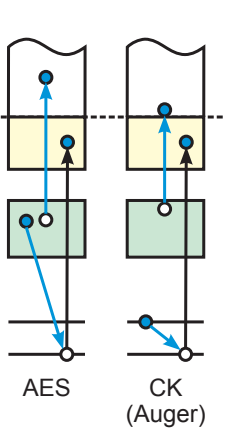

(b)

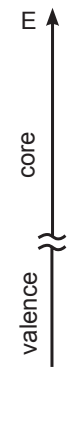

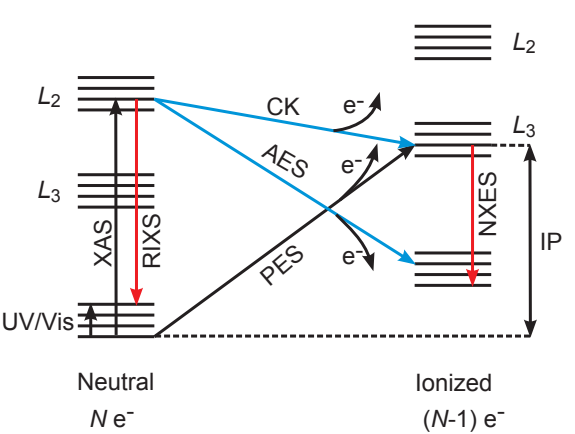

Figure 1: (a) Processes relevant for X-ray spectroscopy viewed from the MO picture standpoint. For absorption, the respective process in UV/Vis range is also shown. (b) Processes relevant for X-ray spectroscopy in the many-body state picture. (Color code for arrows: photon absorption - black, radiative decay of a core hole - red, non-radiative decay - blue). Abbreviations: Photoelectron Spectrum (PES), Non-resonant X-ray Emission Spectrum (NXES), Resonant Inelastic X-ray Scattering (RIXS), Resonant X-ray Emission Spectroscopy (RXES), Auger Electron Spectrum (AES), Coster-Kronig (CK).

For TM compounds, $L$-edge spectra enjoy great popularity $4 \sqrt[4]{5}$ They are due to excitation or ionization from the $2 s$ and $2 p$ orbitals ( $L$-shell) of the target atom to bound or continuum states, respectively. Being interested in frontier $d$-orbitals containing information on the nature of metalligand or metal ion-solvent interaction, dipole-allowed excitation from $2 p$ orbitals is addressed more frequently. Traditionally, solid state samples have been investigated. However, recent developments in the vacuum liquid microjet technique have broadened the applicability of soft X-ray spectroscopy to highly volatile liquid solutions $\underline{39 \mid 40}$

Excitation of a system from its electronic ground state to a high-energy and highly nonequilibrium core-excited state triggers a number of relaxation processes which either represent losses or can be used as a spectroscopic probe of material properties. Let us consider them on the example of $2 p L$-edge spectroscopy as illustrated in Figure1. In general there are two pictures with 
respect to the electronic states that can be used for the discussion. First, the intuitive and therefore widely used "single electron" MO picture (panel (a)). Second, the more appropriate "state picture", which is based in the many-particle eigenstates of the electronic Hamiltonian (panel (b)).

Upon absorption of a soft X-ray photon (0.1-1.0 keV), a $2 p$ core hole is created at the metal center accompanied by $K$-edge ionization of the ligands. This process is depicted on the very left of Figure 1 and is subject of X-ray absorption spectroscopy ${ }^{9}$ In this case, an electron is excited from a localized core MO to delocalized bound one. Within the state picture, the X-ray Absorption Spectrum (XAS) is due to transitions within the $N$-electron manifold, i.e. between the ground and core-excited states of the ("neutral") system.

The core-electron can also be excited into the continuum and the photocurrent of the outgoing electrons can be measured yielding a PES. In the state picture this corresponds to a transition between the initial $N$-electron ("neutral") system and the $N$-1-electron ionized system as illustrated in Figure 1 $\mathrm{b}$. The proper total final state is formed by the antisymmetrized product of the state of the $N-1$ electron manifold and the outgoing free electron state.

The created core-hole can decay either radiatively or non-radiatively. Radiative decay channels comprise $3 d \rightarrow 2 p$ and $3 s \rightarrow 2 p$ pathways, which represent about $1 \%$ of the total decay probability for the first-row TMs $\stackrel{41}{[1}$ Depending on whether the initial excitation has been into a bound or continuum state one speaks about resonant (RXES) or RIXS 42 or the NXES, respectively. The major decay channel is due to Auger, CK decay, and Interatomic Coulombic Decay (ICD) autoionization processes $^{43}$ (Figure 1 13 ). In the state picture these processes involve a transition between the $N$ and $N$-1-electron manifolds and are reflected in the AES.

In general XAS and RIXS are called photon-in/photon-out spectroscopies, whereas PES and AES are photon-in/electron-out techniques. The combination of X-ray absorption and emission together with photoelectron studies provides a very powerful suite of tools to address electronic structure and specific solute-solvent interactions, 5,44

\subsubsection{Absorption spectroscopy}

Absorption spectroscopy provides the most simple type of spectra considered in the context of X-ray excitation as long as the discrete pre-edge structure is considered only. Viewed in the MO picture, XAS probes the unoccupied valence orbitals. Conventional X-ray experiments operate in the weak-field regime ${ }^{9}$ and the respective absorption amplitude, proportional to the absorption cross section at frequency $\Omega$, can be expressed in terms of first-order time-dependent perturbation 
theory (Fermi's Golden Rule)! $\underline{45}$

$$
\mathcal{X}(\Omega)=\sum_{g} f\left(E_{g}, T\right) \sum_{i}\left|\left\langle i\left|\hat{\mathbf{d}} \cdot \mathbf{e}_{i n}\right| g\right\rangle\right|^{2} \Lambda\left(E_{g}+\Omega-E_{i}\right) .
$$

Here, the $|g\rangle$ and $|i\rangle$ states are ground and core-excited final states with respective energies $E_{g}$ and $E_{i}$. Due to finite temperature $T$, there can be several initial states populated according to the Boltzmann distribution $f\left(E_{g}, T\right)=\exp \left(-E_{g} / k T\right) / \sum_{j} \exp \left(-E_{j} / k T\right)$. This especially applies to TM complexes, where the initial high degeneracy of the electronic states can be lifted due to Jahn-Teller effect and SOC, leading to several levels accessible by thermal excitation.

Further, $\hat{\mathbf{d}}$ and $\mathbf{e}_{i n}$ in Eq. 1 are electric dipole operator and polarization vector of the incoming light. The lineshape function, $\Lambda\left(E_{g}+\Omega-E_{i}\right)$, characterizes the density of the final states and, thus, the form of the absorption bands. Effects to be considered here include the width of the incoming excitation pulse, homogeneous (lifetime) broadening due to Auger and radiative decay, and inhomogeneous broadening due to influence of environment, e.g., solvent or phonon broadening in solid state. ${ }^{46}$ These broadening parameters can be often fitted to best reproduce the experiment.

To address XAS theoretically in dipole approximation, one thus needs to calculate energies of the core-excited electronic states with respect to the ground one and the ground-to-core-excited transition dipole moments. Note that for hard X-ray radiation the dipole approximation breaks down and higher order multipole moments have to be considered $\underline{47}$

For $L$-edge spectra, additional complexity enters due to strong SOC, triggered upon core-hole formation and within the $3 d$ states themselves. ${ }^{4}$ This leads to a characteristic shape of the spectrum containing two groups of bands, so-called $L_{3}$ and $L_{2}$ edges (see Figure 4 below).

\subsubsection{Resonant emission spectroscopy (RIXS)}

Staying in the weak-field perturbational regime, the radiative decay of the core-excited state $|i\rangle$ prepared upon absorption of X-ray light can be described within second-order perturbation theory. The respective Kramers-Heisenberg expression for the emission amplitude reads ${ }^{45}$

$$
\begin{aligned}
\mathcal{R}(\Omega, \omega) & =\sum_{g} f\left(E_{g}, T\right) \sum_{f}\left|\sum_{i} \sqrt{\frac{\Gamma_{i}}{\pi}} \frac{\left\langle f\left|\mathbf{e}_{\text {out }}^{*} \cdot \hat{\mathbf{d}}\right| i\right\rangle\left\langle i\left|\hat{\mathbf{d}} \cdot \mathbf{e}_{\text {in }}\right| g\right\rangle}{E_{g}+\Omega-E_{i}-i \Gamma_{i}}\right|^{2} \\
& \times \Lambda\left(E_{g}+\Omega-E_{f}-\omega\right) .
\end{aligned}
$$

It contains the same ingredients as the XAS expression, Equation (1), where in addition $\omega$ and $\mathbf{e}_{\text {out }}$ denote the energy and polarization of the emitted photon, $\Gamma_{i}=1 / \tau_{i}$ is the inverse lifetime of core- 
excited state $|i\rangle$ and state $|f\rangle$ represents the final valence state. Notice that due to the fact that the sum over $i$ appears under the square, the radiative channels ending up in the same final state $f$ interfere with each other. This photon-in/photon-out process represents an electronic analogue of the well-known vibrational Raman spectroscopy. The only difference is that one addresses transitions not between vibrational levels but between different electronic states. Formally, RIXS corresponds to a non-linear $\chi^{(3)}$ process and therefore is analogous to four-wave mixing but with the last two interactions being of spontaneous rather than stimulated nature. $\stackrel{48}{ }$ Thus, it is only linearly proportional to the intensity of the incoming light. Drawing on this analogy the overall spontaneous light emission signal can be separated into two contributions: sequential incoherent fluorescence where a population in the excited state is created followed by radiative decay and a direct "coherent" Raman process. While in the former case resonant excitation is required, RIXS also works for off-resonant excitation, i.e. involving only coherences between the participating states.

In the following we will focus on RIXS only. The RIXS intensity can be recorded as a function of incoming, $\Omega$, and outgoing, $\omega$, photon energy. Thus, it provides a two-dimensional spectrum, resolving both absorption and emission processes. Often one discusses one dimensional cuts at fixed excitation energy of this spectrum only. Alternatively, the so-called Partial Fluorescence Yield (PFY) spectrum at a given excitation energy $\Omega$ can be obtained by integrating with respect to the emission energies in some energy window $\omega \in\left[\omega_{0}, \omega_{1}\right]$

$$
\mathcal{F}(\Omega)=\int_{\omega_{0}}^{\omega_{1}} \mathcal{R}(\Omega, \omega) d \omega
$$

providing a spectrum analogous to XAS. Viewed in the orbital picture (Figure 19), RIXS gives access to the unoccupied MOs as well as to the occupied valence MOs. In state picture (Figure 1 b), absorption and emission processes become coupled because of orbital relaxation due to core-hole and valence excitation relaxation. Thus, RIXS approximately maps the valence excited states. Calculating RIXS is a more elaborate task as compared to XAS since these core-hole and valence excitation states may have different requirements to the computational protocol.

\subsubsection{Photon-in/electron-out spectroscopy}

Photon-in/electron-out spectroscopy represents a powerful suit of methods 25,490 Depending on whether the X-ray photon energy exceeds the binding energy of the core-electron or is below the core ionization threshold one distinguishes between non-resonant PES and Resonant Photoelectron 
Spectrum (RPES), cf. Figure 17. PES provides direct information on the energies of core orbitals, e.g., the value of their SOC splitting, if viewed from the orbital picture perspective. In the state picture, it corresponds to a transition from e.g. ground state of non-ionized system to the coreexcited states of ionized one. Moreover, the shake-up excitations, where ionization is accompanied by valence excitation, can provide valuable insights into the electronic and geometric structure. $\frac{51}{5}$

The resonant counterpart, RPES, contains two processes - direct ionization from the valence level and autoionization of the core-excited state. The former one represents a usual PES where electrons from valence MOs are ejected having kinetic energies comparable to that of the absorbed X-ray photon. The latter one is an Auger process, i.e. a transition between core-excited state of the non-ionized manifold and the valence state of the ionized manifold accompanied by emission of an electron as depicted in Figure 1 b. In terms of orbital picture, it corresponds to core-hole refill by a valence electron and simultaneous emission of another valence electron with high kinetic energy.

The total RPES cross section assuming integration over all directions of the outgoing photoelectron reads

$$
\begin{aligned}
\mathcal{P}\left(\Omega, \varepsilon_{\text {kin }}\right) & =\sum_{i} f\left(E_{g}, T\right) \sum_{f^{+}} \Lambda\left(E_{f^{+}}+\varepsilon_{\text {kin }}-E_{g}-\Omega\right) \\
& \times\left|\left\langle f^{+} \psi^{\mathrm{el}}\left(\varepsilon_{\text {kin }}\right)\left|\mathbf{e}_{\text {in }} \cdot \hat{\mathbf{d}}\right| g\right\rangle+\sum_{i} \frac{\left\langle f^{+} \psi^{\mathrm{el}}\left(\varepsilon_{\text {kin }}\right)\left|\hat{H}-E_{i}\right| i\right\rangle\left\langle i\left|\hat{\mathbf{d}} \cdot \mathbf{e}_{\text {in }}\right| g\right\rangle}{E_{g}+\Omega-E_{i}-i \Gamma_{i}}\right|^{2} .
\end{aligned}
$$

The structure of this expression is a hybrid of first-order Fermi's Golden rule for direct photoionization (first term under square) and second order Kramers-Heisenberg-like term for autoionization. The system starts from thermally populated initial states $|g\rangle$. The incoming X-ray light either ionizes the system by transition to a final state $\left|f^{+} \psi^{\mathrm{el}}\left(\varepsilon_{\text {kin }}\right)\right\rangle$, which is the properly antisymmetrized product of the bound $N$-1-electron ionic remainder state $\left|f^{+}\right\rangle$and the state of the free electron $\left|\psi^{\mathrm{el}}\left(\varepsilon_{\mathrm{kin}}\right)\right\rangle$, having kinetic energy $\varepsilon_{\text {kin }}$. Alternatively, the same state can be obtained by excitation to the intermediate bound core-excited state $|i\rangle$, which then decays non-radiatively, mediated by the Hamiltonian $\hat{H}$ of the system. This decay is essentially due to the Coulomb coupling which is dominating in the respective matrix element.

There are some more comments needed at this point. First of all, due to the coinciding final state of both processes there is an interference between the direct and Auger parts of the signal. This interference is present also for decay channels from different intermediate states in the Auger part of the signal. Second, Equation (4) sheds light on the nature of the parameters $\Gamma_{i}$ entering also the RIXS expression, Equation (2). In fact, $\Gamma_{i}=2 \pi \sum_{f^{+}}\left|\left\langle f^{+} \psi^{\mathrm{el}}\left(\varepsilon_{\mathrm{kin}}\right)\left|\hat{H}-E_{i}\right| i\right\rangle\right|^{2}$, representing 
the total Auger decay rate of core-excited state $|i\rangle$. In principle, radiative decay would add to the total rate, but for TMs autoionization is the dominating process. Next, we note that here we assumed the simple product form $\left|f^{+} \psi^{\mathrm{el}}\left(\varepsilon_{\text {kin }}\right)\right\rangle$ of the final state and thus correlations between the ionic remainder and the outgoing electron are neglected. A more elaborate scheme taking interaction of ionization channels requires a scattering theory formulation. ${ }^{52}$ Finally, RPES can be also viewed as a $2 \mathrm{D}$ type of spectrum ${ }^{44}$ as it depends on the incoming photon energy $\Omega$ and the photoelectron kinetic energy $\varepsilon_{\text {kin }}$.

As compared to XAS and RIXS, PES is analogous to the former and AES to the latter. The difference is that PES contains information on the core-excited states of the ionic remainder, whereas AES contains that of the core states of the non-ionized system and valence manifold of ionized system (see Figure 1b). RPES calculations are more elaborate due to the need to compute integrals between bound and continuum states to obtain matrix elements of the of the dipole moment and molecular Hamiltonian $\hat{H}-E_{i}$.

In the weak field regime, PES cross sections can be calculated in the framework of the Dyson Orbital (DO) formalism.53.55 For illustration, we leave the second term in Equation (4) aside and consider only the direct photoionization represented by the first term. Assuming the strong orthogonality between the state $\left|\psi^{\mathrm{el}}\left(\varepsilon_{\text {kin }}\right)\right\rangle$ and MOs of the initial system, the expression can be rewritten in terms of quasi-one-electron states $\left|\Phi_{f^{+} g}^{\mathrm{DO}}\right\rangle$ which are called DOs. It represents an $N-1$ electron integral over initial and final ionic states $\left|\Phi_{f^{+} g}^{\mathrm{DO}}\right\rangle=\sqrt{N}\left\langle f^{+} \mid g\right\rangle_{N-1}$. Using the DO, the transition matrix element (neglecting polarization dependence) can be expressed as

$$
\left\langle f^{+} \psi^{\mathrm{el}}\left(\varepsilon_{\text {kin }}\right)|\hat{\mathbf{d}}| g\right\rangle_{N} \approx\left\langle\psi^{\mathrm{el}}\left(\varepsilon_{\mathrm{kin}}\right)|\hat{\mathbf{d}}| \Phi_{f^{+} g}^{\mathrm{DO}}\right\rangle_{1}
$$

Here, the subscripts $N$ and 1 denote $N$-electron and one-electron integration, respectively. This formal reorganization allows to separate system and electronic structure method dependence of the intensity from the actual representation of the photoelectron. Once the DO is computed for a particular pair of bound states, $|g\rangle$ and $\left|f^{+}\right\rangle$, it can be further used to estimate transition dipoles for different kinetic energies $\varepsilon_{\text {kin }}$ and even different forms of $\left|\psi^{\mathrm{el}}\right\rangle$. Commonly used free electron functions include the plane wave expansion in terms of partial waves or Coulomb waves. .54 The former is appropriate when photodetachment from a negatively charged species is considered, whereas the latter accounts for spherical Coulomb potential of the ionic remainder. In case of TM complexes, one needs many terms in the expansion to realistically approximate the free electron function. ${ }^{53}$ This approach has facilitated interpretation of experimental XUV and X-ray PESs of 
transition metal complexes in solution $44,56,58$

Finally, we would like to mention the so-called Sudden Approximation (SA) ${ }^{[59}$ It assumes that the cross section for a pair of states, $|i\rangle$ and $|f\rangle$, is proportional to $\left|\Phi_{f i}^{\mathrm{DO}}\right|^{2}$. Thereby the kinetic energy dependence of the cross section is neglected such that no bound-to-continuum integrals in Equation (5) need to be calculated. This approximation is usually considered to be justified if the nature of the transitions and thus of the DOs is similar and kinetic energies are large.

\section{THEORETICAL METHODS}

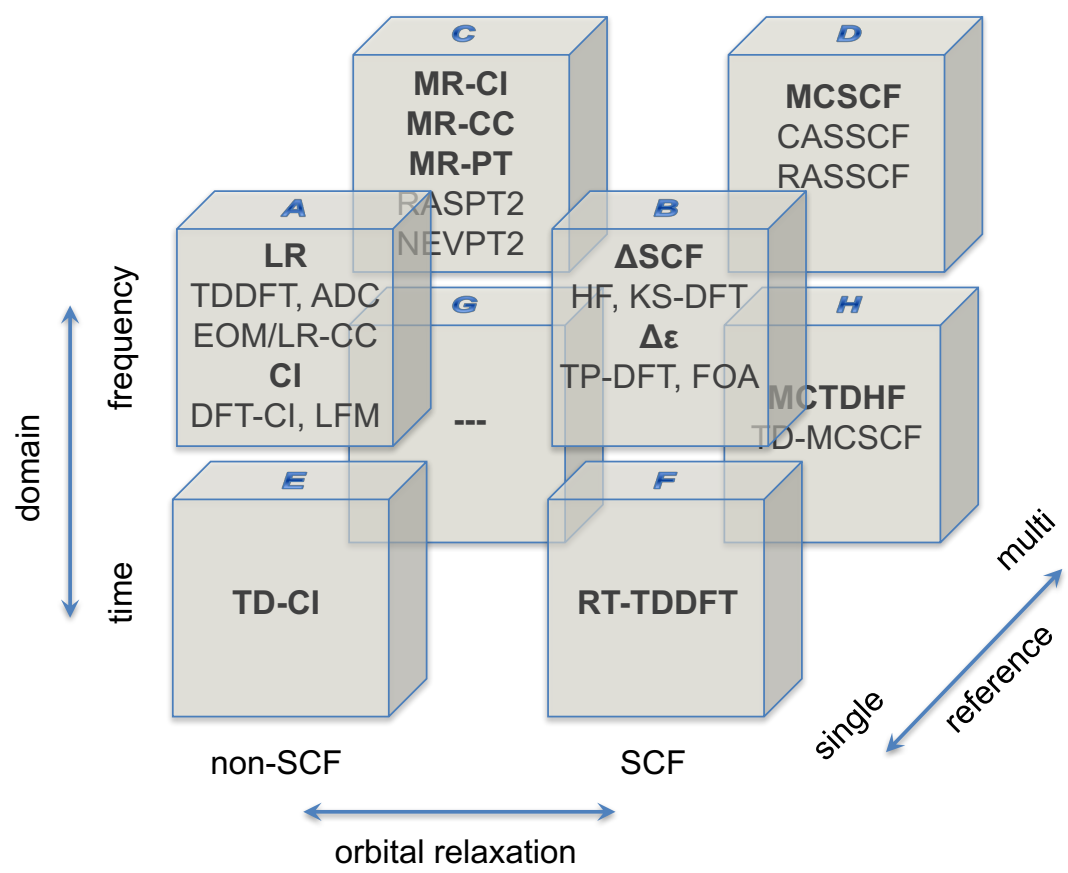

Figure 2: Overview on different methods discussed in this review, grouped according to three criteria: (i) frequency (energy) vs. time domain, (ii) explicit orbital optimization, i.e. SCF vs. non-SCF, and (iii) reference for construction of excited state basis is based on a single or multiple electronic configurations. (MR-CI, MR-CC, and MR-PT are multi-reference CI, CC, and PT, respectively. Other abbreviations are defined in the text.) 


\subsection{General remarks}

Theoretical studies of X-ray spectra can be divided into two classes: 1) investigations of closed-shell molecules containing mainly the second-period atoms addressed with single-reference methods and 2) works reporting on open-shell systems, primarily TM complexes. In general, first principles quantum chemical predictions for excited electronic states of TM compounds are significantly more difficult than for compounds containing main group elements $\frac{60}{6 M}$ chemistry is closely connected to near degeneracy effects, also called static correlation, making methods based on a single SCFlike reference configurations unreliable. Thus, computationally more demanding multi-reference approaches are often required to obtain quantitative accuracy. In addition, the treatment of $d$ electrons requires the inclusion of dynamic electron correlation. Finally, especially for core-excited states, relativistic effects, e.g., SOC, have to be taken into account.

An overview of available methods is provided in Figure 2, In general, corresponding methods can be formulated in time $(\mathrm{E}, \mathrm{F}, \mathrm{G}, \mathrm{H})$ and energy $(\mathrm{A}, \mathrm{B}, \mathrm{C}, \mathrm{D})$ domain, which amounts to solving either the time-dependent or stationary Schrödinger equation. The spectroscopic observable (Equations (1), (2), and (4) are formulated in terms of the eigenvalues and eigenstates of the stationary Schrödinger equation, $\hat{H}|\Psi\rangle=E|\Psi\rangle$. However, the spectra could be equivalently expressed in as Fourier transforms of multi-time dipole correlation functions. ${ }^{45}$ For instance for XAS, these are two-point and for RIXS four-point dipole correlation functions 6164 Beyond this perturbational regime, i.e. for strong field excitation, or in cases of (shaped) ultrashort laser pulses one has to resort to explicit solution of the time-dependent Schrödinger equation including the field-matter interaction. Compared to the Quantum Chemistry (QC) methods, Real-Time (RT) propagation approaches have received less attention so far.

Another, "dimension" of the classification in Figure 2 is with respect to the type of MOs that are used to express the many-electron states. In fact, every calculation starts from an MO optimization; the question is whether it is done within the actual excited state method as well $(\mathrm{B}, \mathrm{D}, \mathrm{F}, \mathrm{H})$ or the MOs are fixed during excited state calculations (A,C,E). Finally, QC methods can be distinguished according to the used reference space into single (A,B,E,F) and multi-configuration $(\mathrm{C}, \mathrm{D}, \mathrm{H})$ approaches. Note that we are not aware of the methods which fall into category G.

In the following, we will review ab initio QC methods, focussing on MCSCF-based methods in frequency domain (Figure 2D and C) as applied to X-ray spectroscopy of TM complexes. However, other methods mentioned in Figure 2 will be briefly discussed as well. 


\subsection{Basic concepts: SCF}

\subsubsection{Hartree-Fock (HF) theory}

The HF method is the most basic MO approach discussed here. Although it is not directly applied to core-level spectroscopies it is central for understanding other methods. It can be characterized by the following points: (i) The $N$-electron wave function $\Psi^{N}\left(\mathbf{r}_{1}, \ldots, \mathbf{r}_{N}\right)$, describing the ground state of the system, is represented as a single Slater determinant $\Phi^{N}\left(\mathbf{r}_{1}, \ldots, \mathbf{r}_{N}\right)=$ $\operatorname{det}\left\{\phi_{a}\left(\mathbf{r}_{1}\right) \phi_{b}\left(\mathbf{r}_{2}\right) \ldots \phi_{c}\left(\mathbf{r}_{N}\right)\right\}$ of one-electron MOs $\left\{\phi_{i}\left(\mathbf{r}_{j}\right)\right\}$ (for inclusion of spin, see Section 3.6). (ii) The optimal set of $\left\{\phi_{i}\left(\mathbf{r}_{j}\right)\right\}$ is searched variationally to obtain $\left|\Phi^{N}\right\rangle=\left|\Phi_{g}\right\rangle$ giving the best possible estimate $E^{\mathrm{HF}}$ of the exact ground state energy $\bar{E}$ under the restriction of (i), see Eq. (6). (iii) The electronic Hamiltonian contains one- and two-electron terms $\hat{H}=\sum_{i} \hat{h}(i)+\sum_{i j} \hat{g}(i, j)$. HF theory is a mean-field approach where the latter are approximated by a one-electron potential $\sum_{i j} \hat{g}(i, j) \approx \sum_{i} \hat{V}^{\mathrm{eff}}(i)$. Matrix elements of this one-electron operator are expressed via Coulomb, $J_{k l}$, and exchange, $K_{k l}$, two-electron integrals as $\left\langle\phi_{k}\left|\hat{V}^{\text {eff }}(1)\right| \phi_{k}\right\rangle=\sum_{l}\left(J_{k l}-K_{k l}\right)$. (iv) The problem is reformulated in terms of a one-electron eigenvalue equation $\hat{f}_{i}\left|\phi_{i}\right\rangle=\varepsilon_{i}\left|\phi_{i}\right\rangle$, yielding orbitals $\phi_{i}\left(\mathbf{r}_{j}\right)$ and their energies $\varepsilon_{i}$. Since the Fock operator, $\hat{f}_{i}$, depends on the orbitals $\left\{\phi_{i}\left(\mathbf{r}_{j}\right)\right\}$ the equation is solved iteratively (SCF scheme). Essentially, this is done via a search for unitary transformations $\hat{U}(\hat{\kappa})=\exp (\hat{\kappa})$ of orbitals which give a minimal ground state energy

$$
E^{\mathrm{HF}}(\boldsymbol{\kappa})=\min _{\boldsymbol{\kappa}}\left\langle\Phi_{g}|\exp (-\hat{\kappa}) \hat{H} \exp (\hat{\kappa})| \Phi_{g}\right\rangle
$$

where $\hat{\kappa}$ is an anti-hermitian one-electron operator. Thus, the orbital variations are parametrized in terms of the elements of the skew-hermitan matrix $\boldsymbol{\kappa}$, which represents $\hat{\kappa}$ in the basis of MOs; one may think of the elements of $\boldsymbol{\kappa}$ as corresponding to pairwise orbital rotations.

In practice, one uses the Linear Combinations of Atomic Orbitals (LCAO) form, where the MOs are represented in an atomic basis, $\left\{\chi_{k}(\mathbf{r})\right\}$,

$$
\phi_{i}(\mathbf{r})=\sum_{k} c_{i k} \chi_{k}(\mathbf{r})
$$

Thus the coefficients $\left\{c_{i k}\right\}$ are variationally optimized.

According to its mean-field nature, HF theory does not take into account correlations due to the electron-electron interaction. Electron correlation is at least a two-body effect and should be distinguished from the one-electron orbital relaxation accounted for via the SCF procedure. Different types of correlations can be identified if we adopt for the moment a classical point of 
view. Two electrons in an atom will try to "occupy" orbits with different radii (radial correlation) or reside on different sides of the nucleus (angular correlation). This type of correlation is called dynamic (or weak) and can be accounted for by allowing $\left|\Psi^{N}\right\rangle$ to have in addition to the leading HF term $\left|\Phi^{N}\right\rangle$ a small admixture of other determinants built on the same set $\left\{\phi_{i}\right\}$ but with different integer occupation numbers (excited determinants). This approach will be considered later in the context of the CI method in Section 3.3.1. There are cases, when a single leading term for $\left|\Psi^{N}\right\rangle$ cannot be found since several of determinants have comparable weights. This more extreme case leads to complete break-down of HF approximation and is referred to as static (or strong) correlation. It will be considered in the framework of MCSCF theory in Section 3.3.2. Finally, one may distinguish differential correlation, when the correlation energy is different in different electronic states.

\subsubsection{Kohn-Sham Density Functional Theory (KS-DFT)}

The KS-DFT method belongs to the same class as HF, see Figure 2 $\mathrm{B}$. The approach is rather different though. Backed by the Hohenberg-Kohn theorems it formulates the energy as a functional of electron density $\rho(\mathbf{r})$ subject to the variational principle. Since $\rho(\mathbf{r})$ is a three-dimensional quantity and thus simpler than the $3 N$-dimensional wave function, it offers distinct benefits from the viewpoint of computational efficiency. In practice, one considers the Kohn-Sham scheme, where a single determinant is postulated for the ground state. The density is determined as $\rho^{\mathrm{KS}}(\mathbf{r})=\sum_{i}\left|\phi_{i}^{\mathrm{KS}}(\mathbf{r})\right|^{2}$, with $\left\{\phi_{i}^{\mathrm{KS}}(\mathbf{r})\right\}$ being the occupied Kohn-Sham MOs. This approach aims at the exact description of a system, adopting a formally non-interacting electron ansatz for the wave function. The "non-classical" terms which constitute the difference between this HF-like solution and the exact one are thus transferred to the one-electron potential $\hat{V}^{\text {eff }}(i)$. The energy functional of the electron density which is variationally minimized reads $E\left[\rho^{\mathrm{KS}}(\mathbf{r})\right]=$ $T^{\mathrm{KS}}\left[\rho^{\mathrm{KS}}(\mathbf{r})\right]+J\left[\rho^{\mathrm{KS}}(\mathbf{r})\right]+E_{\mathrm{el}-\mathrm{nuc}}\left[\rho^{\mathrm{KS}}(\mathbf{r})\right]+E^{\mathrm{XC}}\left[\rho^{\mathrm{KS}}(\mathbf{r})\right]$, containing kinetic energy of non-interacting system, "classical" Coulomb repulsion energy, electron-nuclear attraction energy, and the eXchangeCorrelation (XC) energy $E^{\mathrm{XC}}\left[\rho^{\mathrm{KS}}(\mathbf{r})\right]$ comprising the difference between exact and approximate $\left(T^{\mathrm{KS}}\right)$ kinetic energies, the effect of electron correlation, and exchange interaction. Various approximate $E^{\mathrm{XC}}$ exist and numerous techniques for the construction of this functional have been devised.

All shortcomings of Density Functional Theory (DFT) result from the approximate nature of $E^{\mathrm{XC}}$. Most notable is the so-called self-interaction error. In HF, the interaction of an electron 
with itself is canceled by the exact relation $J_{i i}-K_{i i}=0$. This is, however, not true anymore if approximate XC functionals are considered. This appears to be critical in many respects ${ }^{65}$ and especially for the core-levels of atoms (see Section 3.5 below).

\subsection{3 (Quasi)-one-electron methods for X-ray spectroscopy}

The KS-DFT method has replaced HF in modern applications. Although being also of one-electron nature, it describes independent quasi-particles, implicitly accounting for (dynamic) electron correlation. Both methods can be used to generate a suitable basis of orbitals for subsequent electron correlation treatment by single-reference methods (Figure 2A), such as CI, PT, or CC approaches as discussed below. The choice of a proper orbital basis is more important for core-excited states than for valence-excited ones. The reason is that removing an electron from the core region where the density of electrons is very high significantly influences electrons occupying other shells due substantial changes in the screening of the nuclear charge, which can be associated with an additional polarization potential. $\stackrel{66}{6}$ Thus, most of the orbitals will strongly relax to become more tightly bound in energy and more confined in space. Naturally, this type of orbital relaxation is very important for core ionization or the description of the subsequent NXES; although there might be exception, see Reference 67. This may require to perform an SCF orbital optimization to account for the core-hole excited state to accurately predict X-ray spectra. The simplest way to do so is to obtain relaxed orbitals where the core-excited atom is replaced by the next element from the periodic table. Within the so-called $Z+1$ or equivalent core approach ${ }^{68}$ these orbitals are used for calculations of the parent molecule. Important applications include the interpretation of PESs and thermodynamic data .69

Although both HF and DFT techniques were described in the previous section as purely ground state methods, in principle, they can be applied to excited states as well. In this case, the excited state is also represented as a single Slater/Kohn-Sham determinant analogously to the ground state; this is called $\triangle S C F$ method since the transition energy is be obtained as the difference between respective SCF energies. Its application to core-excited states has been first suggested in Reference 70. Because some of the features of the $\triangle \mathrm{SCF}$ method will be important for the later discussion of MCSCF calculations of core-excited states, more details will be provided in the following.

A major issue for all methods which involve orbital optimization (cf. Figure 2B,D) is the socalled variational collapse. Let us assume that we have setup a wave function with a hole in some core orbital and an additional electron in a virtual orbital and naively perform a variational SCF 
optimization (note that no spatial symmetry is assumed and the spin coincides with that of the ground state). For a randomly chosen initial orbital guess, the virtual orbital hosting an excited electron will be transformed to the core one in a series of unitary transformations at every SCF iteration. In other words, without additional tricks the wave function is likely to collapse to the ground state and core-excited states cannot be accessed. The only reason why such calculations of deep core-hole excitations might converge to the desired local minimum solution is that usually these deep-lying core orbitals have a negligible overlap and couplings with other MOs. For larger molecules and shallow holes this, however, might not work leading to oscillations, divergence, or to collapse to the ground state.

To circumvent this problem, several schemes have been suggested (Figure 2 B). In approach I, one searches consecutively for higher-lying variational solutions, e.g., under the constraint that each next solution should be orthogonal to all previously found, although this additional condition is not always rigorously applied, see below. 71 This might be a tedious procedure if more than a few core-excited states are needed. Alternatively, in approach II, a one-electron orbital basis is constructed in such a way, that the differences of orbital energies give good estimates of the X-ray transition energies $(\Delta \varepsilon$ approach $)$.

Several different schemes along the lines of approach I have been suggested to perform a constrained search. They differ in the construction of occupied and virtual MOs involved in the excitation and in setting their occupation numbers, see Reference 71 and references therein. Exemplarily, one should mention the application of a multiple hole/particle algorithm within orthogonality constrained DFT to the calculation of $K$-edge spectra ${ }^{72}$ Moreover, state-following algorithms can be applied to avoid the variational collapse without explicit setting the orthogonality constraint. Here, one specifies the desired occupation number pattern for the initial guess and attempts to maintain it during SCF iterations. This can be accomplished by applying different kinds of projectors to separate core orbitals from others at every iteration, see Reference 73 and references therein. Alternatively, one can utilize a maximum overlap criterion ${ }^{74}, 75$ to identify the core-hole orbital at each SCF step. In principle, one can also apply conventional SCF subject to a special construction of the initial guess as discussed in References 76,77. Here, in the first step the singly-occupied core orbital is frozen and thus it cannot mix with other MOs via unitary transformations $\hat{U}(\hat{\kappa})$, i.e. one gets a constrained SCF minimum. Next, one uses this partially relaxed wave function to converge to the local energy minimum, corresponding to a fully relaxed core, making use of quadratically converging algorithms. 
In approach II, one aims at obtaining orbitals partially relaxed due to presence of the core hole such that differences of orbital energies, $\varepsilon_{i}$, are a good approximation of transition energies $E_{i}-E_{g}=\left\langle\Phi_{i}|\hat{H}| \Phi_{i}\right\rangle-\left\langle\Phi_{g}|\hat{H}| \Phi_{g}\right\rangle \approx \varepsilon_{i}-\varepsilon_{g}$. Several versions have been suggested, differing in the included fraction of the core hole and excited electron causing orbital relaxation, e.g., full or half of the core hole and full neglect or inclusion of the excited electron $\sqrt{78} \mid 79$ One of the prominent representatives of approach II is the Transition Potential DFT (TP-DFT) method ${ }^{80}$ reviewed in Reference 81. Here one chooses a half-occupied core orbital and neglects the effect of the excited electron. Such a choice of a partial occupation can be viewed from a more general viewpoint of ensemble DFT, $, 82,83$ where the number of electrons in the system varies from $N-1$ to $N$. For the exact $\mathrm{XC}$ potential, the energy between points with different integer particle numbers $E(N-1)$ and $E(N)$ changes linearly with fractional occupation $n$ and the derivative of the energy exactly

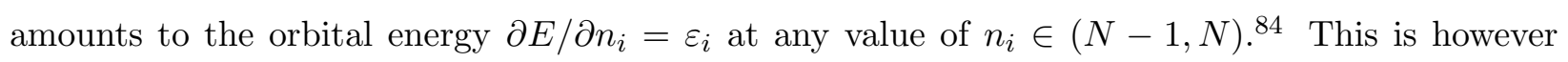
not the case for approximate XC functionals as the energy behaves nonlinearly $\sqrt[85]{ }$ According to Lagrange's mean value theorem the exact relation between the energy derivative and $\varepsilon_{i}$ is restored at some point between $N-1$ and $N$ electron numbers. In a sense, TP-DFT is pretending to search for this optimal point approximating it by $N-1 / 2$. More generally, fractional occupation can be considered as a "fitting" parameter to generate optimal orbitals for X-ray calculations 86,87

Note that in a number of works an even more simplified protocol belonging to approach II, the so-called Frozen Orbital Approximation (FOA), has been used. Here, only ground state SCF is carried out and intensities and energies are fully based on transition matrix elements and energy differences between ground state Kohn-Sham orbitals. FOA is thus completely neglecting excited state orbital relaxation and in this respect it is similar to Koopmans' theorem approach for HF ${ }^{88}$ or

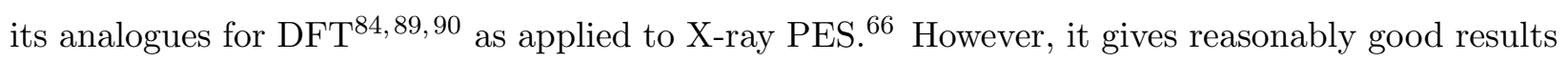
for $K$-edge XAS and NXES of TM compounds $\underline{9192}$

For approach I, the calculation of transition matrix elements is quite complicated in case of nonorthogonal wave functions and can lead to spurious results. Within approach II they are usually estimated on a quite approximate basis as $\mathcal{X}(\Omega) \propto\left|\left\langle\Phi_{i}\left|\hat{\mathbf{d}} \cdot \mathbf{e}_{\text {in }}\right| \Phi_{g}\right\rangle\right|^{2} \approx\left|\left\langle\phi_{i}\left|\hat{\mathbf{d}} \cdot \mathbf{e}_{\text {in }}\right| \phi_{g}\right\rangle \times S\right|^{2}$. Here, $\left|\Phi_{g}\right\rangle$ and $\left|\Phi_{i}\right\rangle$ are SCF solutions for ground and $i$ th core-excited states and $\left|\phi_{g}\right\rangle$ and $\left|\phi_{i}\right\rangle$ are orbitals corresponding to the respective one-electron excitation. Thus, the transition matrix element between $N$-electron states is replaced by a one-electron integral. The factor $S$ is the $N-1$ electron overlap which accounts for the relaxation of other orbitals not involved in the excitation. It is usually assumed to be constant, but can be calculated in a more elaborate way to improve 
computed intensities. 93

The main advantage of the $\triangle \mathrm{SCF}$ method is that one can use the well-developed machinery of ground state SCF theory for obtaining excited state properties without much of new development. One should keep in mind, however, that in case of DFT-based techniques the accuracy of the underlying density functional is of vital importance. $\triangle$ SCF-like methods have been widely applied to the $K$-edges of the second period elements from small molecules $75[94] 95]$ to macromolecules 56 97 and condensed phases 79 81] $83 \mid 98$ It has been even attempted to calculate energies of $L$-edge transitions of $\mathrm{Si}, \mathrm{P}, \mathrm{S}, \mathrm{Cl}$, and $\mathrm{Cu}$ containing systems, ${ }^{75}$ although without proper treatment of relativistic effects like SOC. Finally, $\triangle \mathrm{SCF}$ can also be used to optimize electronic states with multiple holes residing on the core or valence MOs as relevant for Auger spectroscopy and shake-up XAS transitions $.99,100$

\subsection{Methods including correlation}

\subsubsection{Configuration interaction}

Apart from $\triangle \mathrm{SCF}$-like approaches adapting the HF or KS-DFT ground state to compute higherlying states, there is a plethora of single-reference energy-domain excited state methods; cf. Figure $2 \mathrm{~A}, \mathrm{~B}$. The respective excited states can be represented in the general form

$$
\left|\Psi_{i}\right\rangle=\hat{\mathcal{E}}_{i}\left|\Psi_{g}\right\rangle
$$

where $\left|\Psi_{g}\right\rangle$ is the ground state obtained with any suitable method and $\hat{\mathcal{E}}_{i}$ is an excitation operator. In single-reference methods, $\left|\Psi_{g}\right\rangle$ is usually taken to be a single ground state Slater (or Kohn-Sham) determinant, $\left|\Phi_{g}\right\rangle$, and orbitals are not further optimized. We start the discussion of this class of methods with the CI technique, being conceptually the simplest although not computationally most efficient representative. The CI framework facilitates understanding of other (even conceptually different) methods as it provides a very pictorial tool of wave function composition.

$\mathrm{CI}$ is one of the most logical ways to improve on the SCF description $101[102$ First it provides a systematic way for inclusion of electron correlation and second it can be naturally extended to the calculation of excited states. Since ground state and excited Slater (or Kohn-Sham) determinants form a complete basis, a CI state $i$ can be written as

$$
\left|\Psi_{i}^{\mathrm{CI}}\right\rangle=\sum_{j} C_{i j}\left|\Phi_{j}\left(\left\{\phi_{k}^{\mathrm{fixed}}\right\}\right)\right\rangle
$$

Here, the determinants $\left|\Phi_{j}\right\rangle$ are built from the fixed SCF orbitals $\left\{\phi_{k}^{\text {fixed }}\right\}$, obtained prior to the CI procedure, e.g., by ground state HF or KS-DFT. In practice, the coefficient matrix $\mathbf{C}$ is obtained 
by writing the electronic Hamiltonian in the basis of determinants which is iteratively diagonalized using the Davidson algorithm. 103

Using all possible determinants in the expansion Equation (9) corresponds to the exact Full CI (FCI) solution for a given basis set. Its computational complexity grows exponentially with the number of basis functions and truncation schemes are usually applied. Starting point is the classification of the terms in Equation (9) as being unexcited reference, singly, doubly, triply, and so on excited electronic configurations:

$$
\left|\Psi^{\mathrm{CI}}\right\rangle=\underbrace{\left|\Phi_{g}\right\rangle}_{\text {ref. }}+\underbrace{\sum_{i a} C_{i a}\left|\Phi_{i}^{a}\right\rangle}_{\text {singles }}+\underbrace{\frac{1}{4} \underbrace{\sum_{i j a b}\left|\Phi_{i j}^{a b}\right\rangle}_{i j a b}}_{\text {doubles }}+\ldots
$$

In this notation, indices $i, j, \ldots$ and $a, b, \ldots$ denote occupied orbitals from where an electron is excited and virtual orbitals to which electrons are excited in the determinant $\left|\Phi_{i j \ldots}^{a b \ldots}\right\rangle$, respectively. To make the problem computationally tractable, one truncates the expansion Equation 10 including, e.g., all determinants up to a given excitation class. This gives rise to CI Singles (CIS), CI Singles Doubles (CISD) methods, and so on. Since electron correlation is at least a two-body effect, doubles are necessary to correct for it on top of a HF reference. Singles are important for description of charge density and thus for properties like transition dipole moments. If only singles are included, it might provide a good tool for spectroscopy with near-HF quality for the excited states.

From the viewpoint of quantum chemistry the core-excited electronic states are not any different from the valence-excited ones. But, the problem is that one would need to calculate thousands or even millions of states to reach the highly lying levels relevant for X-ray spectroscopy. This problem stems from the fact that, if one is interested in the $n$th eigenvalue, the efficient Davidson algorithm ${ }^{103}$ standardly used for this purpose, diagonalizes at least an $n \times n$ matrix and thus obtains non-interesting eigenvalues below $n$. Since matrix diagonalization is the main computational bottleneck such a brute force approach wastes computational resources if this is possible at all. Let us consider, for instance, two systems $\mathrm{H}_{2} \mathrm{O}$ ( $K$-edge) and $\left[\mathrm{Fe}\left(\mathrm{H}_{2} \mathrm{O}\right)_{6}\right]^{2+}(L$-edge). If one takes a quite moderate triple-exponent TZP basis and accounts for singly and doubly excited configurations which is the minimal reasonable choice to include electron correlation within the CI approach, the total amount of valence states is 46872 and 136370360 , respectively. This means that to reach the first $1 s$ - or $2 p$-core-excited state one would need to explicitly calculate a huge number of states, where in case of XAS often only few low-lying ones are of relevance for the spectrum (due to the factor $f\left(E_{g}, T\right)$ in Equation (1)). 
There are several ways to circumvent this difficulty. First of all, one can stay within the conventional Davidson scheme and project onto a smaller subspace of trial functions excluding most (or even all apart from the ground state) valence states (Figure 3). In practice one makes use of the concept of an Active Space (AS) of MOs, where certain orbitals (i.e. the core orbitals for XAS calculations) are allowed to change their occupations, while for the others electronic excitations are forbidden (also called Core-Valence Separation (CVS) $104-106)$. Equivalently, the subspace of electronic configurations can be limited according to simple Koopmans' estimates of the state energy based on the orbital energies as realized within Restricted Excitation Window Time-Dependent Density Functional Theory (REW-TDDFT) $107 \mid 108$
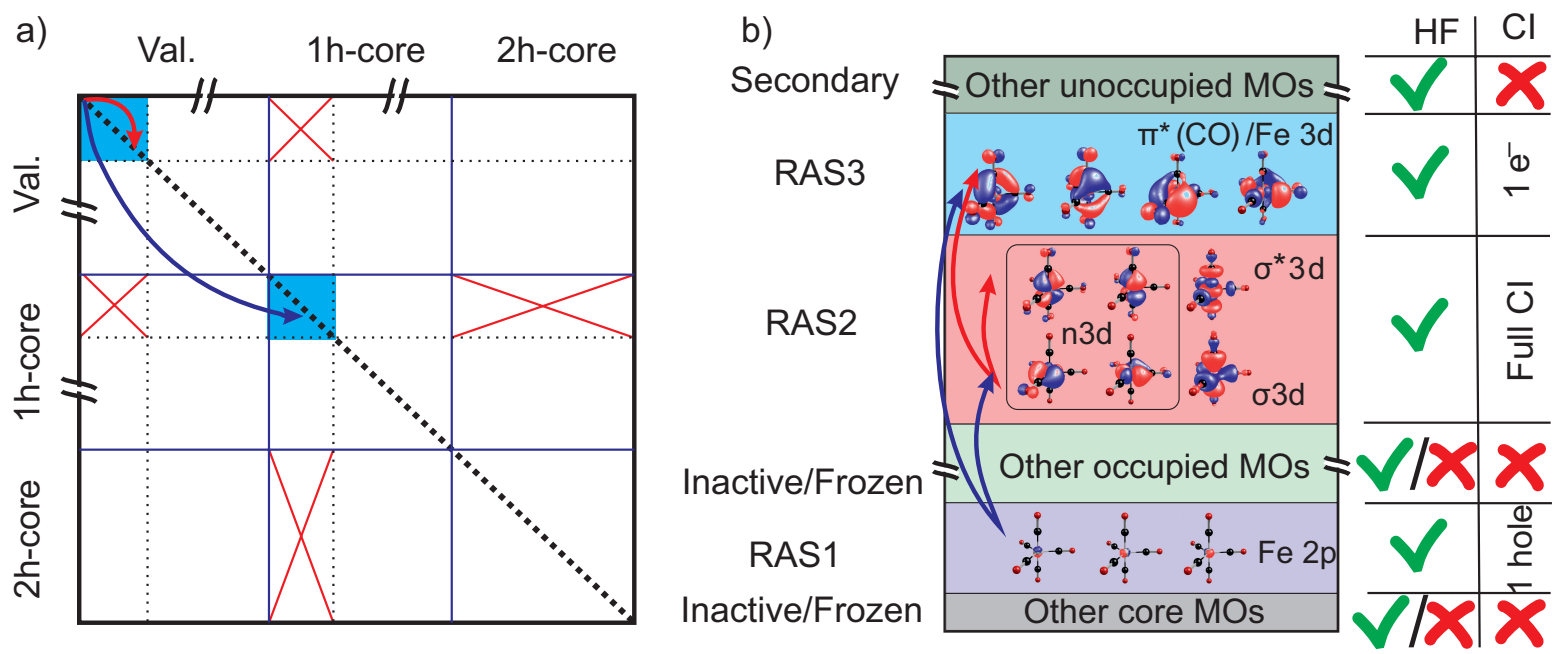

Figure 3: (a) Illustration of the projection of the full Hamiltonian to the subspace of limited valenceand core-excited configurations (blue blocks), neglecting the respective off-diagonal coupling blocks (red crosses). (b) Principal MO subspaces used in the RASSCF method. The typical AS is exemplified for the $\mathrm{Fe}(\mathrm{CO})_{5}$ complex. The table on the right demonstrates the level of orbital optimization (HF) and CI for each subspace. (Valence electronic excitations are depicted by red and core excitations by blue arrows, respectively.)

This "reduced subspace" concept is illustrated in Figure3a. It shows a pictorial view of the CIlike Hamiltonian matrix expressed in the basis of electronic configurations resulting from valence, single core-hole (1h-core), and double core-hole (2h-core) excitations. The overall dimensions of the Val-Val block are those thousands or millions mentioned before. In fact, in XAS one is usually interested in relatively small sub-block (marked with blue color) of the 1h-core-1h-core block including only few tens, hundreds or even thousands of states if only the discrete pre-edge region is 
of interest. In case of RIXS, one needs in addition a number of low-lying valence-excited states also denoted as a blue sub-block of the Val-Val block. The 2h-core-2h-core block is usually completely neglected. The same holds for the coupling Val-1h-core and 1h-core-2h-core blocks. The motivation for neglecting off-diagonal couplings between two configurations from $v$ (valence) and $c$ (core) is provided by PT. Here, the error is estimated to be of the order of $V_{v c} /\left(E_{c}-E_{v}\right)$ in the wave function and $\left|V_{v c}\right|^{2} /\left(E_{c}-E_{v}\right)$ in energy, where $V_{v c}$ is the off-diagonal Hamiltonian matrix element and $E_{c}-E_{v}$ is the usually large difference between energies of these configurations standing on the matrix diagonal. In fact, the restricted subspace approach is equivalent to neglecting specific type of two-electron integrals of the form $\langle a b \mid c d\rangle$, where three of the orbitals $a, b, c, d$ are valence and one is core orbital or vice versa and where orbitals in the bra are both core and in the ket both valence. $104-106$ Hence the diagonalization of the enormously large CI matrix in Figure $3 \mathrm{a}$ can be replaced by a Davidson diagonalization of the small (blue) sub-blocks. The uniform error introduced by CVS was estimated to be of the order of $0.5-1.0 \mathrm{eV}$ for $K$-edges of the second period elements. $109[110$

Another way to access core-states is to modify the Davidson algorithm itself. For instance, in Reference 111 , a modification of the standard algorithm 103 employing the so-called "root-homing" technique has been suggested. It utilizes the maximum overlap criterion (similar to $\Delta \mathrm{SCF}$ ) to identify the desired state during each Davidson iteration step. A more recent modification 112 builds on the idea of Reference 113 and introduces an intermediate projection of the matrix to be diagonalized onto the subspace of the trial vectors having energies in a predefined window. After diagonalization the eigenvectors are projected back onto the full space. The approximate energy of the trial vector is estimated as the orbital energy difference. This approach is superior to the simple index fixing used in REW-TDDFT ${ }^{107}$ as it systematically includes contributions out of the initially predefined reduced space in a consistent way. This gives a good approximation for the high-lying states in the full space of trial vectors without explicit calculation of the lower-lying ones. For the high density of states typical for X-ray spectra, a more advanced adaptive hybrid algorithm can be applied to improve the convergence!114 Apart from CI,111]115 so far such approaches were realized for Linear-Response TDDFT (LR-TDDFT) 112]114|116 and Equation-Of-Motion Coupled Clusters (EOM-CC) ${ }^{117}$ methods discussed below.

In the truncated CI ansatz, Equations $(9)$ and $(10)$, the underlying one-electron orbitals determine accuracy and convergence of the CI expansion. That is why the choice of the orbital basis is of considerable importance for the overall efficiency. It is known that virtual MOs from ground state 
HF calculations are too diffuse especially for large basis sets and thus represent a poor choice. The suggested remedies are to use improved virtual orbitals, $\frac{118}{18}$ natural orbitals, $\frac{119}{19}$ or even KS-DFT orbitals.120 In Reference 87, different types of the relaxed initial orbitals (restricted, unrestricted, and restricted open-shell HF and KS-DFT) have been considered and their performance for coreexcitations within CIS is deduced by comparison to experiment. It was found that a HF reference where orbitals are relaxed to the core-hole is most suitable. Ground state frozen orbitals are not that good and produce errors of order of $10 \mathrm{eV}$ in core excitation energies.

CI has been traditionally used for predicting X-ray spectra due to $K$-edge transitions in small molecules 111$][115 \mid 121] 122$ For $L$-edge spectra of TM complexes, the Configuration Interaction on KS-DFT orbitals (DFT-CI) approach enjoys great popularity. In this method, the standard CI $\hat{\mathcal{E}}^{(i)}$ operator is applied to the ground state KS-DFT determinant $\left(\left|\Phi_{\mathrm{KS}}\right\rangle\right) ![123$ The idea is to take the dynamical correlation effects at the level of DFT into account and to incorporate stronger (static) correlation effects by CI. As a result, the number of important determinants and thus the size of the Hamiltonian matrix can be substantially reduced. This method largely relies on the better suitability of Kohn-Sham orbital energies as estimates of the electronic transition energies. Since these orbital energy differences are dominating the diagonal of the CI Hamiltonian matrix in the basis of determinants, it makes the major contribution to the final CI energies ${ }^{124}$ However, since the CI is built on top of quasi-particle orbitals which already implicitly include dynamic electron correlation a number of points have to be considered. First, double counting of correlation effects should be excluded which can be done by energy-dependent exponential scaling of the CI couplings between determinants. ${ }^{125}$ Moreover, the two-electron integrals calculated on KS-DFT MOs are overestimated and need to be scaled down even in case of CIS, where no double counting occurs due to its "uncorrelated" character $124[126$ The need to parametrize this scaling makes the method semiempirical in nature although the number of parameters is kept as small and universal/transferable as possible. The parameters determined, e.g., by least-squares fitting to experiment, depend on many aspects: XC functional, multiplicity and character of the target excited states, and so on. Thus, the variants usually applied to valence TM spectroscopy 127 need to be adjusted to address core spectra, e.g., by changes of the virtual orbital energies.124

The hybrid nature of DFT-CI provides another distinct advantage making use of the correlated origin of the Kohn-Sham MOs. For TM complexes with covalently bound ligands it reproduces the effect of bonding better than HF which predicts bonds to be in general too ionic $[60$ There are two variants of DFT-CI which were applied to X-ray spectra of TMs. In their approach, Ikeno et al. 
study crystalline metal oxide phases within a cluster approach.128 133 No corrections for doublecounting or scaling of two-electron integrals is performed. As an XC functional the simplest local density approximation is applied, thus containing no exact exchange. Note that the CI problem is solved only in small subspace of MOs containing few electrons (essentially $2 p^{6-n} 3 d^{m+n}$, where $n=0,1$, and also $2 p$ ligand orbitals to account for charge-transfer effects) and the other electrons and their interaction with active ones are taken in an averaged approximate way 134 This makes this approach being strongly related to the Ligand Field Multiplet (LFM) semi-empirical method described below, although no fitting of parameters beyond usual DFT level is performed. Despite of the absence of fitting parameters and the approximations introduced, this method demonstrates a remarkable level of agreement with experimental XAS and other types of spectra. A similar CI approach based on state-averaged relativistic Dirac-Hartree-Fock orbitals (no DFT) has been applied to X-ray $4 f$ and $2 p$ PESs of $\mathrm{UO}_{2} \sqrt{135}$ and $\mathrm{MnO}, \frac{136}{12}$ respectively.

The second DFT-CI method specifically designed to calculate TM L-edge spectra is DFT-based Restricted Open-shell Configuration Interaction Singles (ROCIS)! 126 The restricted open-shell SCF calculation ensures the proper reference ground state configuration with unpaired electrons which is typical for TM complexes. Being a DFT-CI technique, it needs rescaling of two-electron integrals to additionally reduce electron-electron interaction similar to LFM. In practice, three parameters which are universal across the periodic table are introduced: two scaling Coulomb and exchange terms on the diagonal of the CI matrix and one common scaling parameter for all off-diagonal elements. With this it puts emphasis on the diagonal of the CI matrix containing basically the excitation energy for a given orbital pair. These scaling coefficients are optimized only for two density functionals: B3LYP and BHLYP in a specific def2-TZVP(-f) atomic basis. Thus, this method is semi-empirical making use of a training set containing experimental spectra. From the viewpoint of CI it accounts only for single electron excitations thus being intrinsically of SCF quality for the excited electronic states and not including any additional electron correlation on top of KS-DFT MOs. Although being a relatively crude approximation, it allows to keep the configuration space very compact at the price of neglecting shake up excitations. Therefore, it can significantly underestimate the density of states in energy regions where such satellite bands are occurring. On the other hand, this approach is computationally very efficient, especially in connection with a local correlation treatment!137 It has been applied to models including up to several tens of TM atoms. $\frac{138}{13}$ This method is one-component (see Section 3.6) but scalar relativistic effects and SOC are included what is prerequisite to reproduce $L$-edge X-ray spectra. Overall, 
this approach provides fairly good agreement with XAS experiments at least for highly-symmetric systems. 126

Finally, we briefly mention LFM theory as another semi-empirical CI-like method. It has played an important role in understanding the complex structure of X-ray spectra during the last three decades. Within the MO paradigm, the covalent bonding situation is usually described as a oneelectron effect resulting in mixing of orbitals of bound atoms. LFM follows more the valence bond theory route, where these effects are taken on the level of many-electron CI between configurations with localized electrons.

LFM theory has its origin in ligand field theory developed back in $1950 \mathrm{~s}, \frac{139}{13}$ but there are several modifications to accommodate the finer spectroscopic features of TM complexes. It is an atomic theory focussing on an isolated metal ion with, e.g., a $3 d^{n}$ configuration (corresponding to an electronic state $\left|3 d^{n}\right\rangle$ ). The in general complicated chemical environment is taken into account as a perturbation via an effective electric field that disturbs the spherical symmetry of an atom 140,141 Since the effect of ligands goes beyond effective electrostatic interaction especially in case of highly covalent metal-ligand chemical bond, this model needs to be significantly extended. The major extension is to incorporate sophisticated bonding contributions in terms of charge-transfer between metal and ligand. $\frac{142}{14}$ For this purpose in the framework of X-ray spectroscopy, one needs to consider not only $\left|2 p^{6} 3 d^{n} L^{0}\right\rangle$ and $\left|2 p^{5} 3 d^{n+1} L^{0}\right\rangle$ electronic configurations with "neutral" ligand $\left(L^{0}\right)$ and either fully filled $2 p$-shell $\left(2 p^{6}\right)$ or having one core hole $\left(2 p^{5}\right)$, but also states where an electron is transferred, e.g., from the ligand to the $3 d$-shell $-\left|2 p^{6} 3 d^{n+1} L^{+}\right\rangle$and $\left|2 p^{5} 3 d^{n+2} L^{+}\right\rangle$.

If one extends the number of basis functions in which the LFM Hamiltonian is written to the size of a few or even tens of thousands electronic configurations, upon diagonalization one obtains a complex structure of eigenvalues called multiplets. These multiplets originate from the manifold of atomic levels, which are additionally split in energy due to the ligand field and charge-transfer effect.

Often, LFM theory serves as a model for semi-empirical fitting of a number of parameters to reproduce experiments (for an overview see Reference 4). The Coulomb and exchange interactions within core and valence manifold as well as between them are parametrized via Slater-Condon integrals precalculated at the HF level and tabulated for each element. Additionally, their numerical values are often reduced to $80 \%$ or even smaller, to roughly account for electron correlation. The $3 d$ valence and the $2 p$ core SOC is also introduced semi-empirically as a parameter within $L S$ or $j j$ coupling limits (see below) depending on the TM ion considered. Another parametrized 
input for the Hamiltonian is the ligand field. In case of high symmetry TM complexes, e.g., octahedral or tetrahedral, only one effective parameter is used. However, the number of ligandfield parameters grows when symmetry is lowered. Finally, the charge-transfer parameters are adjusted to fit experimental results. The overall number of adjustable parameters can become quite large, thus complicating the fitting procedure. Due to its semi-empirical nature this theory represents a valuable tool to postanalysis of experiments but has only limited predictive power. The transition energies are usually calculated with an accuracy not better than $1.0 \mathrm{eV}$ but in some cases an impressive agreement with experiment has been achieved. $[4$

To parametrize more complicated ligand fields the necessary information can be taken from ab initio calculations. The tandem of the simplicity of LFM theory with the versatility of first principles approaches gave a rebirth to the well-established concept ${ }^{132}$ Moreover, band structure codes can be utilized for such a parametrization in case of solids 143,144

\subsubsection{Multi-Configurational Self-Consistent Field method}

Multi-configurational methods have been introduced to describe bond dissociation, distorted geometries, near-degenerate ground states, and electronically excited states. During decades of application, these methods have acquired a reputation of being the "golden standard" for this type of problems. ${ }^{145]}+153$ From the 1980's on, when the first efficient and rapidly converging algorithms for MCSCF have been developed, also $K$-edge core-level spectra for diatomics and small polyatomic molecules have been calculated $76\left[\begin{array}{ll}754 & {[55}\end{array}\right.$ During the last years investigations of $L$-edge X-ray spectra of TM compounds emerged, where it has been shown to be able to cope with highly excited states in multiconfigurational situations. $156-158$

The MCSCF method is a hybrid of the HF and CI approaches. It employs the CI multideterminantal ansatz, Equation (10), for the many-body state,

$$
\left|\Psi_{i}^{\mathrm{MCSCF}}\right\rangle=\sum_{j} C_{i j}\left|\Phi_{j}\left(\left\{\phi_{k}^{(i)}\right\}\right)\right\rangle
$$

with the important difference that not only the CI coefficients, $C_{i j}$, are variationally minimized, but also the set of coefficients $c_{k l}^{(i)}$, within the LCAO ansatz

$$
\phi_{k}^{(i)}(\mathbf{r})=\sum_{l} c_{k l}^{(i)} \chi_{l}(\mathbf{r})
$$


The MCSCF wave function can be parametrized as

$$
\left|\Psi_{i}^{\mathrm{MCSCF}}\right\rangle=\exp \left(\hat{\kappa}^{(i)}\right)\left(\sum_{j} C_{i j}\left|\Phi_{j}\left(\left\{\phi_{k}^{(i)}\right\}\right)\right\rangle\right)
$$

where operator $\exp \left(\hat{\kappa}^{(i)}\right)$ describes an orthogonal transformation of a set of MOs $\left\{\phi_{k}^{(i)}\right\}$ which are specific to state $|i\rangle \stackrel{150}{1}$ The energy functional can be expressed as

$$
E_{i}^{\mathrm{MCSCF}}(\mathbf{C}, \boldsymbol{\kappa})=\min _{\mathbf{C}, \boldsymbol{\kappa}} \frac{\sum_{j k} C_{i j} C_{i k}\left\langle\Phi_{j}\left|\exp \left(-\hat{\kappa}^{(i)}\right) \hat{H} \exp \left(\hat{\kappa}^{(i)}\right)\right| \Phi_{k}\right\rangle}{\sum_{k} C_{i k}^{2}} .
$$

Upon joint optimization of $\mathrm{CI}$ and $\mathrm{MO}$ coefficients, the one-electron MO basis adapts to the description of electron correlation effects. This allows to quite reliably account for static correlation (multiconfigurational character) and (near-)degeneracies of the electronic states, which are typical for TM compounds.

However, this simultaneous treatment of orbital relaxation and electron correlation leads to a considerable increase in complexity of the actual simulations. The number of CI configurations and MO coefficients quickly increases with the number of atoms and the number of basis functions per atom. The straightforward application of Equation (11) considering all orbitals on equal footing is rather demanding if not impossible already for medium-sized systems. There have been attempts to individually select the most important configurations, e.g., via perturbation theory $159 \sqrt[160]{1} \mathrm{~A}$ more robust and popular solution is provided by the concept of the AS. At the most general level it corresponds to the disjoint subsets of MOs for which different levels of CI expansions are performed. Historically, the most popular variant is the Complete Active Space SCF (CASSCF) approach, $\frac{161}{\mathrm{cf}}$. Figure 3 b. Here, the orbitals are classified in three groups: (i) those which stay doubly occupied are called inactive, (ii) those which are unoccupied, virtual or secondary, and (iii) active ones, forming a subspace where all possible distributions of some number of active electrons are allowed, thus corresponding to a subspace FCI calculation. It is important to note that inactive orbitals are treated at the HF level and that is why the scaling with the physical size of the system is not prohibitive. The exponential growth of the computational demands can be thus mitigated or even avoided by treating only the local correlated subsystem within the AS. For fine tuning with respect to important configurations, one can introduce additional subspaces restricting the number of electrons which can be excited from or to them. This leads to the RASSCF method $162[163$ if the number of active subspaces is equal to three or, in general, to the Generalized Active Space SCF (GASSCF) method $164\left[165\right.$ or similar approaches. ${ }^{166}$ 
To sum up, MCSCF unites the benefits of the SCF and CI allowing not only for orbital relaxation upon core-hole formation but also for strong correlation effects. Therefore, it optimally suits the treatment of TM systems which are well-known for static correlations. Moreover, as we have seen for the case of CI for core-level calculations, the most pragmatic and robust approach to coreexcited states employs restricted subspaces of orbitals and excitations between this subspaces. This naturally leads to the idea of using CASSCF or RASSCF for X-ray spectroscopy. It should also suit to probe with X-rays the excited state dynamics involving bond cleavage or conical intersections, being a native habitat of MCSCF-based methods.

The choice of included orbitals in the AS fixes electron configurations considered within CI treatment. This has to be done in a balanced way as in general correlation effects do not naturally split into strong and weak ones but continuously fill the range of importance. Thus, the selection of the AS has been for a good while considered to be an art. As of now there is a consensus on how to select a more or less optimal AS in general non-pathological cases $150[167-170$ The AS should comprise: (i) orbitals which are involved in the excitation to a particular state $i$, (ii) all orbitals hosting unpaired electrons, (iii) strongly correlating orbitals - usually respective bonding and antibonding MO pairs, (iv) MOs which are responsible for near-degeneracy, e.g., $\sigma$ and $\sigma^{*}$ if the chemical bond is dissociating or degenerate $d$-orbitals for TMs or $f$-obritals for lanthanides and actinides, (v) more specifically in case of $3 d-\mathrm{TMs}, 3 s$ and $3 p$ orbitals might be included for the elements in the beginning of a row to include semicore correlation. For the later elements of a row, additional set of $4 d$ orbitals is required to recover radial dynamic correlation of the electrons in the non-bonding $d$-orbitals already in the AS (double-shell effect). Note that some of the rules might be redundant since, for instance, $3 d$ electrons are strongly correlating and at the same time respective orbitals can be singly occupied and degenerate. Naturally, to perform a computationally feasible calculation, the number of active MOs as well as active electrons needs to be kept as small as possible.

On the one hand, core-excited states can be considered as quite difficult from the viewpoint of selection of AS since the number of $d$-electrons changes in course of excitation/deexcitation or (auto)ionization. On the other hand, in practice this does not represent a problem. Exemplarily, let us consider, how to choose the AS for a core state RASSCF calculation of the $\mathrm{Fe}(\mathrm{CO})_{5}$ model system,$\frac{18}{18}$ see Figure $3 \mathrm{~b}$. Although $\mathrm{Fe}(\mathrm{CO})_{5}$ has a high symmetry, it is not obeying atomic selection rules. However, X-ray excitation involves a very localized part of the electronic system, having predominantly atomic nature. Thus, we can still use approximate angular momentum $(\Delta l= \pm 1)$ 
selection rules. Therefore, to study dipole allowed $L$-edge transitions one needs to include at least $2 p$ and $3 d$ orbitals into the AS as illustrated in Figure $3 \mathrm{~b}$. The AS is further subdivided into RAS1, RAS2, and RAS3 subspaces. Usually, one is interested in singly core-excited states whereas doubly core-hole states are not relevant (Figure 33). To account for this and simplify the calculation, $2 p$ orbitals are put in a separate subspace RAS1. Further, only one electron is allowed to be excited from RAS1 to other active orbitals, meaning that RAS1 contains 6 (for valence states) or 5 (for core states) electrons distributed in the three $2 p$ orbitals. The $3 d$ electrons are usually highly correlated what especially applies to $\mathrm{Fe}(\mathrm{CO})_{5}$ being a covalent complex.169 Therefore, one would prefer to include them in a separate subspace, RAS2, and construct FCI-type configurations. Apart from the doubly-occupied non-bonding $n 3 d$ orbitals which reside almost exclusively on the Fe atom, there is a pair of bonding/antibonding $\sigma 3 d$ and $\sigma^{*} 3 d$ orbitals which are essential for correlation treatment. Moreover, one of the two main transitions in XAS is due to the $2 p \rightarrow \sigma^{*} 3 d$ excitation and in RIXS the $\sigma 3 d \rightarrow 2 p$ relaxation plays an important role $\frac{18}{18}$ Additionally, one can include ligand orbitals, e.g., in the RAS3 subspace and limit the number of electrons which are allowed to be excited to them from RAS1 and RAS2, e.g., to one. It is reasonable to include $\pi^{*}(\mathrm{CO}) / \mathrm{Fe} 3 d$ orbitals which have a mixed $\pi^{*}(\mathrm{CO})$ and non-bonding $3 d$ character. These orbitals are involved in the $2 p \rightarrow \pi^{*}(\mathrm{CO}) / \mathrm{Fe} 3 d$ transitions giving rise to an intense absorption feature $\frac{18}{\text { These empty }}$ ligand-dominated orbitals can be included to describe effects of backdonation and explicit charge-

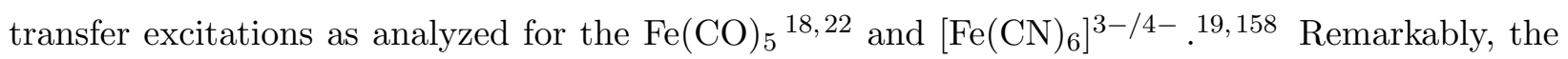
radial nodal structure of these MOs resembles that of the "double-shell" $4 d$ orbitals. Thus, being correlated, or in other words allowing for higher than single excitations to RAS3, they might help to describe radial $3 d$ correlation. However, if one focuses on reproducing transition strengths somewhat sacrificing accuracy in energy, single excitations should suffice ${ }^{18}$ If one is interested in other charge-transfer effects, one might add additional orbitals to RAS1 or RAS3 spaces. All other occupied orbitals are set inactive and stay always doubly occupied.

The complexity of the selection of the AS for TM compounds strongly varies with the covalence of the metal-ligand bond. One can state that covalent complexes correspond to particularly strong static correlation, whereas ionic complexes can be essentially singly-configurational 168,169 In case of ionic complexes $\left[\mathrm{M}\left(\mathrm{H}_{2} \mathrm{O}\right)_{6}\right]^{n+}$, the AS can be kept even more compact including just three $2 p$ and five $3 d$ orbitals ( $t_{2 g}$ non-bonding and two $e_{g}$ anti-bonding orbitals in octahedral symmetry) $21,44\left[53[157,171]\right.$ Other filled orbitals with $3 d$ character, for instance, the bonding $e_{g}$ counterpart, can be added to describe further shake-up and charge-transfer transitions as exemplified for, e.g. 
$\left[\mathrm{FeCl}_{6}\right]^{3-158}$ and $\left[\mathrm{Mn}\left(\mathrm{H}_{2} \mathrm{O}\right)_{6}\right]^{2+/ 3+}$ complexes. ${ }^{21}$ Inclusion of $4 s$ orbitals into the AS accounts for allowed $2 p \rightarrow 4 s$ excitation, having, however, lower intensity without notable influence on $L$-edge spectra $\frac{157}{16}$ It might be still important for accurate reproduction of experimental spectra 172

As a byproduct of having both filled and unfilled (or half-filled) valence MOs in the AS one obtains a fraction of valence electronic states. In fact, states with valence $3 d \rightarrow 3 d$ character can be used to calculate RIXS and RPES. That is why, one should always take care that not only core but also bright valence states (in a sense of core $\rightarrow$ valence radiative relaxation) are represented appropriately. This condition is automatically fulfilled for the AS shown in Figure $3 \mathrm{~b}$. Finally, by choosing the AS one can switch on and off various effects, e.g., in addition to $3 d \rightarrow 2 p$ back relaxation one can account for dipole-allowed $3 s \rightarrow 2 p$ decay by simply adding $3 s$ orbitals to the AS $\underline{44}$

As a note in caution: Imposing constraints on orbital subspaces does not in general case solve the problem of variational collapse. The matrix $\kappa$ in Equations (13) and (14) contains terms which are interchanging active MOs with inactive and virtual ones. Effectively, those orbitals, which correspond to the largest coupling elements in the Hamiltonian, will stay in or enter the AS. Thus, there is always a probability that the calculation will not converge to the desired local minimum of the energy (with core orbitals in the AS) but to the global one, where core MOs are rotated out of AS. As mentioned already for the case of single-reference SCF, if the initial orbital guess is reasonable then, due to small overlap of $2 p$ orbitals with the valence ones and using at least a second order algorithm, the initial orbitals will tend to stay active and the system will fall into the closest local minimum of energy. In our experience, this procedure works quite well for high-spin states if the orbitals optimized for the lowest valence excited states are used as a starting guess. Nevertheless, a systematic convergence to the desired local minimum cannot be ensured and often fails for medium- and low-spin electronic states of TM complexes. A possible solution might be to use a core-hole relaxed initial guess as suggested by Ågren et al 776

Non-desired rotations can also be suppressed by restricting the elements of $\boldsymbol{\kappa}$ mixing $2 p$ orbitals with others to zero, i.e. to perform a constrained SCF search. This approach is justified by the findings in References 66, 173, i.e. that a hole in some deep-core shell does not influence much other orbitals in the same shell and thus relaxation of $2 p$ orbitals can be neglected (see also References 76,77 ). In addition, one can utilize symmetry arguments in case of centrally symmetric systems. 174

Another "brute force" solution is to freeze all occupied orbitals in the inactive subspace, while 
still allowing for rotations between RASn subspaces and virtual orbitals. ${ }^{157}$ This approach still includes some orbital relaxation due to possibility to "borrow" some orbital contributions from the virtual space and redistribute them among active MOs. Although it sounds quite restrictive, in fact, it produces results which agree with experiments fairly well as illustrated in Section 4 . This approach allows to efficiently compute a few thousands of the electronic states which are needed to cover all relevant valence and core levels of the neutral and ionized systems as depicted in Figure $1 \mathrm{~b}$.

In fact, doing an MCSCF calculation produces non-orthogonal electronic states since MOs for different states $i$ are not orthogonal with all the negative implications for the calculation of the transition properties. It is connected to notable numerical effort as the $(\mathbf{C}, \mathbf{c})$ set needs to be determined for each electronic state separately, whose number for core-excited states can easily reach few thousands. To circumvent this and improve convergence properties, the state-averaging procedure is routinely employed ${ }^{[177}$ Within this approach the averaged ensemble density and energy over several electronic states are obtained via variational minimization of the functional

$$
E_{\mathrm{av}}=\sum_{i} w_{i} E_{i}^{\mathrm{MCSCF}}, \quad\left(w_{1} \geq w_{2} \geq \ldots \geq w_{N}\right)
$$

with respect to a common set of orbital rotations. According to the most general formulation of the respective variational theorem by Gross-Oliveira-Kohn, $\frac{178}{1}$ the weighting coefficients should not increase with the state number. In practice, the weights $\left\{w_{i}\right\}$ are usually taken to be equal. Thus, the index $i$ for the set of orbitals in Equation (13) can be omitted as they are the same for all states $\left|\Psi_{i}^{\mathrm{MCSCF}}\right\rangle$ in the ensemble. Having a common set of MO coefficients, a more balanced description of several states is achieved and the difference in energies is resembled by the CI. Such a procedure allows to obtain a set of orthogonal non-interacting electronic states. However, via averaging, the orbitals loose their optimality for the CI-expansion. Nevertheless, averaging allows to attain quite good agreement with experiment. The state averaging for core states has been suggested by McWeeny $173[179$ and used to interpret results of X-ray Photoelectron Spectrum (XPS) and Auger spectroscopies.

On the one hand, due to the large number of core-excited states which usually need to be accounted for, state averaging is a highly advantageous procedure as it reduces the computational effort. On the other hand, if the optimal orbitals for different states are substantially different, this state-averaging procedure becomes not eligible. One might expect such a situation if a number of valence and core states are optimized simultaneously since the former require no relaxation upon the influence of the core hole and the latter do. (A separate averaging of valence and core states 
has been also suggested [180) The unexpected practical solution to this problem is that even if one does democratic averaging over all the states obtained within a given AS, the results agree well with experiments. The democratic average can be viewed as accounting for non-integer corehole occupation somewhat similar to the TP-DFT approach. Thus, the orbitals represent some compromise between needs of valence and core states ${ }^{[19}$ In passing we note that this reminds on the procedure where all the irrelevant lower-lying states are averaged with the interesting higherlying ones to improve convergence and mitigate variational collapse 177

\subsubsection{Restricted Active Space Perturbation Theory (second order) (RASPT2)}

The description of electron correlation within MCSCF can be considered as unbalanced since it accounts for near-degeneracy effects (static correlation) but in addition also partially for the dynamic correlation within the AS. The MOs outside the AS are treated at the HF level with the respective moderate costs, see Figure $3 \mathrm{~b}$. A more balanced description requires a more complete account for the dynamic correlation contribution and can be achieved via PT applied on top of MCSCF. Here, one considers the correlation in an approximate way due to, for instance, single and double excitations between inactive, active, and virtual orbital subspaces in case of PT up to second order. The second order energy correction reads

$$
E_{i}^{\mathrm{MR}-\mathrm{PT}}=-\sum_{j} \frac{\left|\left\langle\Phi_{j}|\hat{V}| \Psi_{i}^{\mathrm{MCSCF}}\right\rangle\right|^{2}}{E_{j}-E_{i}^{\mathrm{MCSCF}}+\delta},
$$

where $\left|\Psi_{i}^{\mathrm{MCSCF}}\right\rangle$ is the MCSCF state, Equation (13), to be corrected by PT, and configurations $\left|\Phi_{j}\right\rangle$ with respective energies $E_{j}$ correspond to the single and double excitations mentioned above, $\hat{V}$ is the difference between the true Hamiltonian and its zero-order approximation, and $\delta$ is the

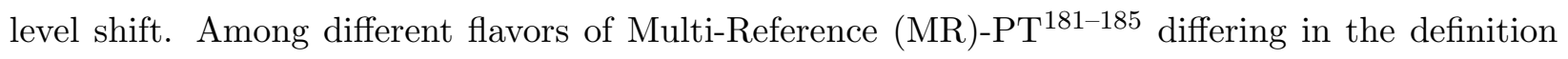
of the zero-order approximation, Complete Active Space second order PT (CASPT2) 186 [187] and its restricted variant RASPT2 $2^{163}$ have enjoyed popularity for excited states of TM complexes in general $167[169] 188$ as well as for TM $L$-edge X-ray spectra. A concise introduction to this method listing main peculiarities and inherent problems can be found in Reference 189; for a more detailed discussion see also References 167, 188 .

At this point we would like to highlight two issues: (i) CASPT2 or RASPT2 cannot correct for the effect of a too small AS. In fact, usually a larger AS than the minimal one is required to get accurate PT results. This is reflected in the AS selection rules listed above, for instance, the necessity to include "double shell" $d$-orbitals. In principle, this effect can be mitigated by 
using the multi-state version of RASPT2 ${ }^{190}$ Here, the effective Hamiltonian is constructed in the basis of single-state RASPT2 wave functions and subsequently diagonalized giving a more precise description. However, it might be impractical for core states due to their large number.

(ii) The energy expression in Equation (16) can be subject to singularities due to the vanishing denominators, giving rise to the so-called intruder state problem. This is especially severe for core-excited states which can easily get degenerate with some $\left|\Phi_{j}\right\rangle$ configuration. The natural solution is to increase the AS if possible. Another solution is to apply a real-valued level shift $\delta$ (Equation (16) ), 191, 192 which artificially lifts the degeneracy with the unwanted weakly-interacting intruder states. An even more elegant solution is the introduction of purely imaginary level shifts, 193 which is in fact utilized for the core-level computations. Nevertheless, choosing a universal level shift which is good for a large number of states at the same time might be problematic and one has to stay at the RASSCF level. Perhaps the best solution is known as $N$-electron Valence second order PT (NEVPT2) 183 [184 It explicitly includes two-electron terms in the zero-order Hamiltonian and is manifested to be intruder state-free. However, it has not yet been widely applied to X-ray spectra calculations, except the work in Reference 172 .

Finally, we note that the effect of RASPT2 depends on the covalence of the complex, $\frac{169}{16 i t h}$ relatively small effect for aqueous metal ions $\$ 2156$ but large impact for systems, like metal hexacyanides, where the affordable AS is not big enough to include all important ligand-dominated MOs. 174

Recently, the RASSCF/RASPT2-based multi-reference approach to X-ray spectroscopy of metal complexes was shown to be quite efficient in unraveling the nature of different transitions in static

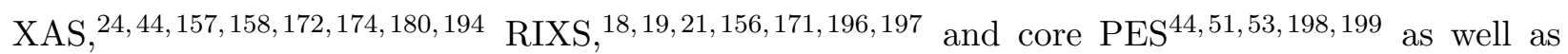
dynamics studies $.22[31,56,200$ Selected examples will be presented in the Section 4 .

\subsection{Time-domain approaches}

A conceptually different approach is the direct solution of the Time-Dependent Schödinger Equation (TDSE) (for a review, see Reference 201) employing a time-dependent Hamiltonian, $\hat{H}(t)=\hat{H}_{\text {mol }}+$ $\hat{V}_{\text {int }}(t)$, with the field-free molecular Hamiltonian $\hat{H}_{\text {mol }}$ and the molecule-external field interaction $\hat{V}_{\text {int }}(t)$. The state $|\Psi(t)\rangle$ can be written in some time-independent basis, e.g., $|\Psi(t)\rangle=\sum_{i} a_{i}(t)\left|\Phi_{i}\right\rangle$, with the temporal evolution recast in the form of time-dependent coefficients. This expansion can be done on the level of orbitals, leading to the time-dependence in MO coefficients. It results in the TD-SCF method, e.g., Real-Time Time-Dependent Density Functional Theory RT-TDDFT 
in case of the KS-DFT ansatz for the wave function. If one assumes time-independent electronic configurations (e.g., Slater determinants) built on time-independent MOs allowing CI coefficients evolving in time one gets the Time-Dependent CI (TD-CI) method. Uniting both approaches results in the Time-Dependent MCSCF (TD-MCSCF) technique, 202 which in the most general form can be also viewed as an Multi-Configurational Time-Dependent HF (MCTDHF) method!203

The advantage of solving the TDSE is that core-excited states can be accessed right away by choosing the proper frequency of the oscillating external field. Further, for pulsed excitation one can tune the width of the frequency window to be investigated 204 Besides focusing on the absorption of light, shaping the envelope of the pulse opens a way to discuss the excitation of intricate superpositions of eigenstates and quantum control of the system's dynamics in real time.

Various real time methods have been applied for simulations of the core spectra and dynamics in the core-excited states such as RT-TDDFT, 204 206 TD-CI, 207-209 non-Dyson Algebraic Diagrammatic Construction (ADC), 210 real-time time-dependent Equation Of Motion (EOM)-CC,211 and $\rho$-TD-RASCI.200.212, 213

\subsection{Response function methods}

While real time-domain propagation methods are emerging, perturbative response function approaches enjoyed considerable popularity over the last decades. Since it falls outside the main scope of the review, we will briefly discuss non-SCF single-reference methods only (for an extensive review, see Reference 12). Response approaches are based on a perturbation expansion of the time-dependent observables in frequency domain, 214 $\sum_{\omega_{1}} R^{(1)}\left(\omega_{1}\right) E\left(\omega_{1}\right) e^{-\mathrm{i} \omega_{1} \mathrm{t}+\gamma \mathrm{t}}+\ldots$, where an external monochromatic field $E\left(\omega_{1}, t\right)=E\left(\omega_{1}\right) e^{-\mathrm{i} \omega_{1} \mathrm{t}}+$ c.c. is assumed. In lowest order only the term linear in the field is retained and the limit $\gamma \rightarrow 0$ provides the basis for Linear Response (LR) methods. For instance, the absorption spectrum is obtained from the Fourier-transformed first-order response or correlation function $R^{(1)}\left(\omega_{1}\right)$ where the operator $\hat{O}$ is the dipole moment operator. Moreover, by using correlation functions involving different operators one can generate the full realm of various propagator methods. ${ }^{215}$ The main advantage of the so-called polarization propagator methods and closely related Green's function approaches ${ }^{216}$ is that the spectrum is determined directly from poles and residues of the propagator. This allows to avoid explicit calculation of the initial and final states and thus to minimize the error in differential correlation.

Among the propagator methods the ADC scheme ${ }^{217}$ and in particular its second-order variant 
$\mathrm{ADC}(2)$ has been actively developed recently. As far as core level calculations are concerned, including CVS, it goes back to the 1980's104|105 with early applications to X-ray spectra of small molecules reported in References 109, 110,218, More complex recent applications have been reported

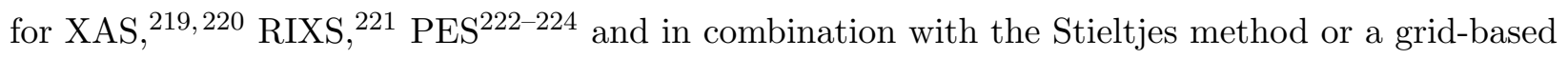
representation of the ionized electron to AES and ICD 225.226

The most widely used response function approach is LR-TDDFT whose versatility in predicting $K$-edge spectra of molecules containing main group elements is well documented, for a review see Reference 10 . In the $K$-edge case, the relevant electronic states are essentially single-configurational and thus can be safely treated by a single-reference method. Being related to the CIS method,227 LR-TDDFT needs to incorporate the same tricks as discussed for the CI method. These include configuration space or energy window restriction $107 \cdot 108$ or modification of the diagonalisation procedure itself leading to an energy specific solver $112[114] 116$

LR-TDDFT is prone to problems due to the so-called self-interaction error. It correlates with the overlap between the occupied and unoccupied orbitals, corresponding to an electronic transition.228 This overlap is very small for core-to-valence and especially for core-to-Rydberg transitions $^{229}$ due to different radial sizes of very confined core and notably more delocalized virtual valence and Rydberg orbitals. Consequently, the energies of core excitations are usually substantially underestimated by LR-TDDFT with standard XC-functionals. ${ }^{230}$ Similar to charge-transfer valence-valence transitions, 231 energies of core transitions were found to be sensitive to the amount of exact exchange in the XC-functional 116$] 230$ Here, exact exchange refers to orbital-dependent exchange integrals, analogous to the HF $K_{k l}$ term, but calculated using Kohn-Sham orbitals. 65.232 As a remedy, functionals with an adjusted amount of exact exchange have been suggested, such as $\mathrm{BH}^{0.57}$ LYP for carbon $K$-edge transitions ${ }^{230}$ and the CV-B3LYP for $K$-edges of second period elements, where core regions are described by BHHLYP (50\% HF exchange) and valence with B3LYP (20\% HF exchange) ${ }^{233}$ Another, more gentle way is to split the Coulomb operator into short-range and long-range parts depending on the interelectron distance and switch exact exchange between different values with a smooth function. Most important for core spectroscopy is the short-range correction as introduced in References 229, 234, leading to a remarkable improvement of $K$-edge transition energies. For other correction methods designed for prediction of X-ray spectra, see also References 235237 .

LR-TDDFT has been successfully applied to main group element $K$-edges $\frac{10,112}{116}[238$ demonstrating good accuracy and predictive power. Attempts have also been made to calculate $L$-edges 
of TMs $\frac{107[108|204| 239}{2 n}$ In particular, the REW-TDDFT method has been used to demonstrate different kinds of non-linear X-ray spectroscopies such as stimulated RIXS to probe the dynamics of electronic and nuclear wave packets in the excited states.240.241

Another way to treat correlation on top of SCF is provided by the Coupled Cluster (CC) technique. In contrast to the linear form of the CI expansion, it employs an exponential ansatz for the excitation operator such that the ground state wave function can be obtained, e.g., from the HF solution as $\left|\Psi_{g}\right\rangle=\exp (\hat{T})\left|\Psi_{\mathrm{HF}}\right\rangle$. The operator $\hat{T}=\hat{T}_{1}+\hat{T}_{2}+\ldots$ generates singly, doubly, and so on excited configurations similar to the CI method. One also does a hierarchical truncation according to excitation level as in the truncated CI discussed above. Its immense success for ground state studies $^{242}$ suggests an extension to the excited electronic states.

The CC approach can be generalized to treat excited states within the $\mathrm{EOM}^{243}$ or $\mathrm{LR}^{244}$ formalisms, giving the same energies but different transition moments. ${ }^{245}$ The advantage over conventional CI is that the excited state wave function is not a linear combination of the determinants but has an exponential counterpart inherited from the reference state. Essentially, it can be obtained from Equation (8), where $\left|\Psi_{g}\right\rangle=\exp (\hat{T})\left|\Psi_{\mathrm{HF}}\right\rangle$ and $\mathcal{E}_{i}$ has the same structure as in the CI case. The operator $\exp (\hat{T})$ effectively accounts for higher-order excitations due to its exponential form and $\mathcal{E}_{i}$ treats differential correlation with respect to the ground state.

Both EOM and LR-CC have been applied to $K$-edge core spectra recently. For example, EOM$\mathrm{CC}$ with singles and doubles excitations has been applied on top of the HF reference with a core-hole obtained with maximum overlap method for emission spectroscopy of water.246 Alternatively, the energy specific algorithm, as discussed in Section 3.3.1, was used together with EOM-CC for a set of molecules containing main group atoms. $\frac{117}{17}$ Different approaches have also been used to get core-ionized potential energy surfaces in case of the ClF molecule ${ }^{247}$ In Reference 248 , a two-step approach is utilized, describing separately the effect of core-hole relaxation and an excited bound electron by means of electron-attachment variant of EOM-CC. A simpler reduction of the excitation space is introduced for the LR-CC method $249 \mid 250$

The EOM-CC and LR-CC methods fail if the ground state cannot be described by a singlereference $\mathrm{CC}$ wave function. In contrast to $\mathrm{CI}$, the extension of the $\mathrm{CC}$ idea to the multi-reference case is non-trivial due to the problem with definition of a proper vacuum state leading to a number of technical complications, for review see Reference 251. However, various flavors of multi-reference CC have been applied to core-spectra of small main group molecules recently $252 \sqrt{254}$ 


\subsection{Relativistic effects}

Relativistic effects are traditionally considered for the valence chemistry of heavy elements. $255 \sqrt[256]{2}$ However, they also play an important role for core electrons as they experience the (almost) unscreened Coulomb potential of the nucleus. For instance, these effects make $s$ and $p$ core electrons even more tightly bound to nuclei resulting in an additional stabilization of the $s$ and $p_{1 / 2}$ levels and destabilization of the $p_{3 / 2}, d$, and $f$ ones. $\frac{257}{25}$ This adds different energetic shifts, e.g., to $L_{3}$-edge $\left(2 p_{3 / 2} \rightarrow 3 d\right)$ and $L_{2}$-edge $\left(2 p_{1 / 2} \rightarrow 3 d\right)$ transitions. Further, SOC is determining the characteristic shape of $L_{2,3}$-edge spectra.

A rigorous treatment of relativistic effects is provided by the Dirac equation, for reviews see References 258 263. Here, the wave functions are four-component vectors, corresponding to positive (electronic) and negative (positronic) energy states. Four-component theory, equipped with a proper electron correlation treatment, is without doubt the "golden standard" of accuracy in relativistic calculations. But, the considerable computational costs limit their applicability to atoms or small (highly-symmetric) molecules. Examples of four-component core calculations include (i) DFT-CI based calculations of $\mathrm{MO}_{6}^{n-}(\mathrm{M}=\mathrm{Ti}, \mathrm{Fe}, \mathrm{Ni} \text {, etc })^{128} \frac{132}{12}$ and $\mathrm{UO}_{8}^{12-135}$ clusters, (ii) LRTDDFT calculations of $K$ - and $L$-edges of $\mathrm{H}_{2} \mathrm{~S}^{264}$ and $\mathrm{Si}$, Ge and S halides, $\stackrel{265}{2}$ and (iii) ADC-based investigations of $\mathrm{XeF}_{n} N$-edge spectra ${ }^{266}$ as well as of photoionization of $(\mathrm{HI})_{2}$ and $(\mathrm{LiI})_{2} 2^{267}$ and of $\mathrm{MnO} \underline{136}$

Accounting for negative energy states contributes little to the physical and chemical processes most relevant for molecules containing no very heavy elements. This situation is reflected by the fact that in case of low and medium nuclear charge $Z$ there are solutions of the Dirac equation having dominant contributions from positive energy states. Various techniques have been designed to decouple both components such that the small component is implicitly included in calculation of the large component. This can be done in principle within any predetermined level of accuracy yielding so-called exact two-component methods. $\stackrel{268}{2 x a m p l e s ~ i n c l u d e ~ t h e ~ Z e r o-O r d e r ~ R e g u l a r ~ A p-~}$ proximation (ZORA) 269 and Infinite-Order Two-Component (IOTC) methods, $\stackrel{270}{ }$ which are often used for core-level calculations. The transition from four- to two-component theory can also be done via the Douglas-Kroll-Hess protocol271]272 which applies a sequence of unitary transformations eliminating the coupling order-by-order and being exact in the limit of an infinite number of transformations. This yields a block-diagonal Hamiltonian represented as a perturbational sum, where in addition to block diagonalization the spin-dependent and spin-free parts can be separated, 
thus building a basis for distinguishing scalar and, e.g., SOC relativistic effects. In practice, however, one transforms only one-electron terms as the transformation of two-electron integrals is very involved and is often sacrificed for the sake of efficiency. This might lead to inaccuracies in $L_{3} / L_{2}$ splittings as discussed in Reference 206.

Such a block-diagonalization of the Dirac Hamiltonian with subsequent neglect of the negative energy block leads to two-component schemes. Here, SOC is usually taken into account at the stage of the variational SCF orbital optimization leading to the so-called $j j$-coupling limit. The justification behind is that for high- $Z$ elements SOC becomes comparable to the largest two-electron integrals and hence significantly influences the one-particle MO basis. Examples of 2-component calculations include (i) ZORA DFT-CI based calculations of oxides, $\frac{133}{\text { (ii) ZORA LR-TDDFT }}$ calculations of $K$ - and $L$-edges of Ti complexes, $273 \mid 274$ of chemical shifts in $K_{\alpha 1,2}$ emission lines of $\mathrm{YbF}_{n} \stackrel{275}{275}$ and $\mathrm{Nb}$ oxide, $\stackrel{276}{ }$ XAS of gas phase TM oxochlorides, $\stackrel{277}{ }$ short-chain oligothiophenes XAS and XPS 238 (iii) real-time DFT calculations of XAS for TM chlorides and oxochlorides, 206 (iv) RASSCF IOTC calculations of XPS of noble gases. $\stackrel{278}{27}$

For compounds containing low and medium $Z$ elements the most pragmatic approach is to neglect the spin-dependent part of the Hamiltonian in the two-component scheme, resulting in a one-component approach. It corresponds to the usual solution of the Schrödinger equation with the difference that scalar relativistic effects are taken into account. The latter have been shown to be crucial for predicting the correct energetics of X-ray spectra $126|264| 279$ Typically, scalar relativistic effects are treated at the second-order Douglas-Kroll-Hess level.

For $L$-edge spectra the magnitude of SOC is larger than typical energy level spacings and thus it must be taken into account. Within the one-component protocol SOC is included as a perturbation of the spin-free problem in the framework of the so-called quasi-degenerate perturbation theory ${ }^{280}$ Here, the SOC Hamiltonian, $\hat{H}_{\mathrm{SOC}}$, is represented in the basis of spin-free spatial wave functions multiplied by an appropriate spin part. The SOC-coupled eigenenergies and eigenstates are obtained via matrix diagonalization and have the form of linear combination of spin-free states, $\left|\Psi_{n}^{\mathrm{SF},\left(S, M_{S}\right)}\right\rangle$, with complex coefficients

$$
\left|\Psi_{a}^{\mathrm{SOC}}\right\rangle=\sum_{n} \xi_{a n}^{\left(S, M_{S}\right)}\left|\Psi_{n}^{\mathrm{SF},\left(S, M_{S}\right)}\right\rangle
$$

In practice for core-level calculations, it is advisable to use as many states for the zero-order state basis set $\left\{\left|\Psi_{n}^{\mathrm{SF},\left(S, M_{S}\right)}\right\rangle\right\}$ as are available for a given AS ${ }^{174}$ Note that since in this approach not the total momenta of the individual electrons are coupled as in the $j j$-limit, but rather the total 
angular momenta $L$ and spins $S$ of many-body electronic states, it is referred to as $L S$-coupling limit.

The expectation values and transition matrix elements are then calculated for the SOC-coupled eigenstates $\left\{\left|\Psi_{a}^{\mathrm{SOC}}\right\rangle\right\}$. Therefore, formally spin-forbidden transitions may gain notable intensity in X-ray spectra which is borrowed from the allowed ones via SOC-mixing,21|44]|157 In passing we note that this mixed nature of SOC eigenstates is also reflected in the structure of the DOs, which become complex orbitals having spin-up and spin-down components due to mixing of different multiplicities. 53

For such an a posteriori treatment of SOC the operator $\hat{H}_{\mathrm{SOC}}$ in the Breit-Pauli form ${ }^{281}$ is commonly used. It can be represented in terms of one-, $\hat{h}_{\mathrm{SOC}}(i)$, and two-electron, $\hat{g}_{\mathrm{SOC}}(i, j)$, operators As usual, multi-center two-electron terms require the most effort in the calculation of $\hat{H}_{\text {SOC }}$ matrix elements in the basis of spin-free electronic states. However, they cannot be neglected (especially for light elements) without notable decrease in accuracy.

Let us consider a matrix element of $\hat{H}_{\text {SOC }}$ between two Slater determinants differing in the occupation of the $i$ th and $j$ th orbitals, whereas occupations of other orbitals, $\left\{n_{k}\right\}$, are the same, i.e.

$$
H_{i j}=\left\langle i\left|\hat{h}_{\mathrm{SOC}}(1)\right| j\right\rangle+\frac{1}{2} \sum_{k} n_{k}\left[\left\langle i k\left|\hat{g}_{\mathrm{SOC}}(1,2)\right| j k\right\rangle-\left\langle i k\left|\hat{g}_{\mathrm{SOC}}(1,2)\right| k j\right\rangle-\left\langle k i\left|\hat{g}_{\mathrm{SOC}}(1,2)\right| j k\right\rangle\right]
$$

An efficient mean-field type approximation is to fix the occupation numbers $\left\{n_{k}\right\}$ to, e.g., ground state HF values. ${ }^{282}$ Further, a speedup is achieved when the molecular mean-field is approximated by the superposition of the mean-fields of the constituent atoms. Accounting for the locality of SOC, decaying much faster with distance than the Coulomb interaction, and the fact that the largest couplings are expected for core orbitals which are close to the respective nucleus, one can stay with one-center two-electron terms only. This completely separates molecular SOC into additive atomic contributions. The $\left\{n_{k}\right\}$ are standardly calculated by the HF method for neutral atoms. This approximation is called Atomic Mean-Field Integrals (AMFI) ${ }^{283}$ and appeared to be quite accurate. ${ }^{280}$ Numerous applications in the field of X-ray spectroscopy confirmed that this technique provides remarkable accuracy not only for valence but also for core-electrons as is evidenced by the prediction of $L_{2} / L_{3}$ energy separations and general shape of XAS, XPS, and RIXS. It is the basis of the SOC treatment in the ROCIS-DFT method ${ }^{126}$ and of all multi-reference calculations discussed in Section 4 .

Finally, we comment on the atomic basis sets which can be employed for core-level calculations. 
The usual way of implicit inclusion of relativistic effects via core-potentials ${ }^{284}$ is naturally not suitable for this purpose. In this approach the core electrons are removed from the respective atoms, essentially simplifying the nodal structure of the MOs, and their effect is accounted by an effective pseudopotential and only transitions between valence levels can be rigorously calculated. However, there have been reports of two-component calculations using pseudopotentials with an a posteriori restoration of the core nodal structure 275,276 However, in general utilization of allelectron basis sets is prerequisite for explicit calculations of X-ray spectra. Moreover, basis sets need to contain very tight functions to describe the core region; to increase the accuracy one might even de-contract otherwise contracted core functions. ${ }^{\sqrt[156]{6}}$ For this purpose, basis sets specifically designed for correlation and at the same time relativistic treatment from the families cc-pwCVXZ-DK ${ }^{285}$ and ANO-RCC ${ }^{286}$ are usually utilized. For calculations of core spectra of compounds containing atoms from the first three periods the IGLO bases have also been developed. 287

\section{APPLICATIONS}

\subsection{Unraveling the electronic structure at the metal-solvent interface}

Solvated transition metal ions have been shown to provide a model system for metal-ligand interactions and in particular for the electronic structure at the metal-solvent interface $44 \mid[157 \mid 171$ In Figure 4 XAS and RIXS spectra for the prototypical ferrous $\left[\mathrm{Fe}\left(\mathrm{H}_{2} \mathrm{O}\right)_{6}\right]^{2+}$ complex are shown. This weak-field high-spin (quintet) complex has $d^{6}$ configuration and a Jahn-Teller distorted tetragonal symmetry. The $d_{z^{2}}$ and $d_{x^{2}-y^{2}}$ orbitals of the iron ion form bonding $\sigma 3 d$ and antibonding $\sigma 3 d^{*}$ orbitals of $e_{g}$ symmetry with the $a_{1}$ orbitals of the water molecules. The other $3 d$ orbitals of $t_{2 g}$ symmetry form $\pi$-type MOs with the water $1 b_{1}$ and $1 b_{2}$ orbitals, where the mixing is notably weaker as compared to the $\sigma$ orbitals. That is why they can be denoted as non-bonding $n 3 d$ MOs. Note that symmetry labels assume octahedral symmetry for simplicity.

Calculated and measured absorption spectra are in rather good agreement as shown in Figure $44.44\left[171\right.$ Focusing on the $L_{3}$ edge, analysis of the core-excitations reveals that almost all intense transitions are of $2 p \rightarrow \sigma 3 d^{*}$ type, but with substantial admixture of $2 p \rightarrow n 3 d$ character. Notice that this mixing of $\sigma 3 d\left(e_{g}\right)$ and $n 3 d\left(t_{2 g}\right)$ orbitals strongly depends on the considered ion. For $\mathrm{Fe}^{3+}$, for instance, it has been found that the low-energy part of the $L_{3}$ edge is dominated by $t_{2 g}$-type transitions. $\frac{157}{157}$ The multiplicity of the core-excited states strongly depends on energy $200 \mid \frac{212}{213}$ For energies up to about the $L_{3}$ maximum spin-conserving quintet-quintet transition dominate. The 
region up to $715 \mathrm{eV}$ is characterized by strongly mixed quintet-triplet transitions. The $L_{2}$-edge is comprised essentially of spin-forbidden quintet-triplet transitions, which borrow intensity by mixing with spin-allowed ones.

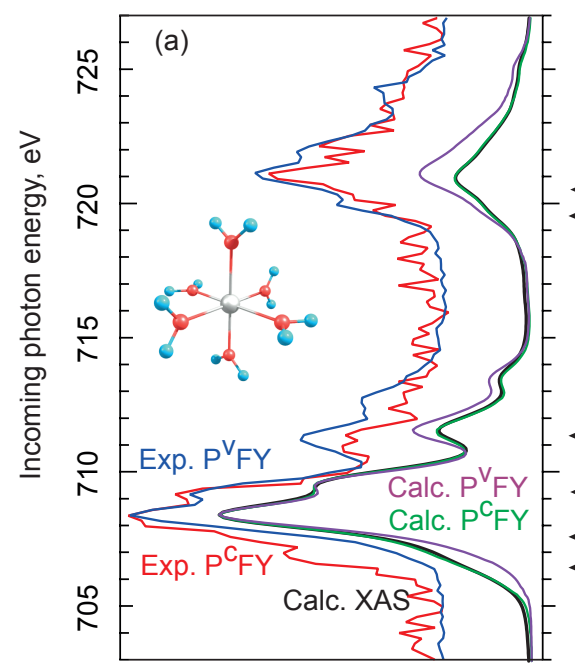

PFY intensity, arb. un.

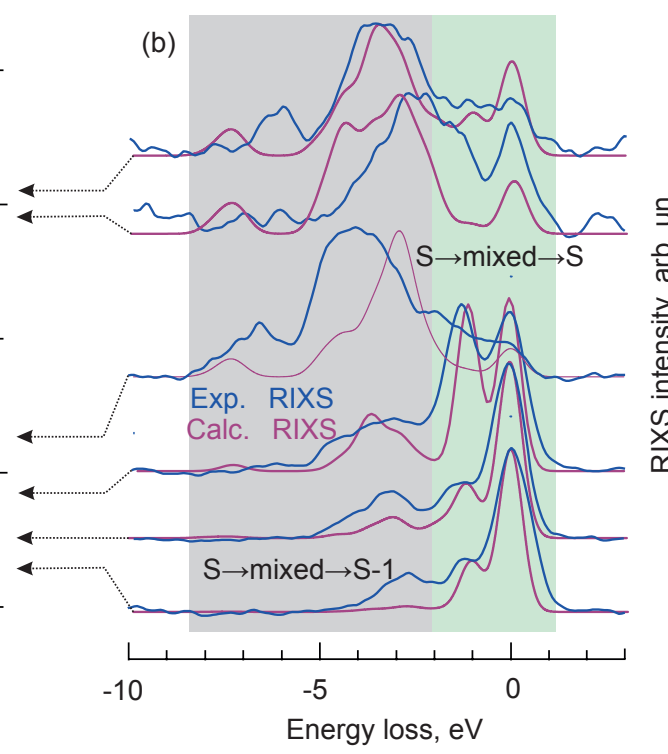

(c)
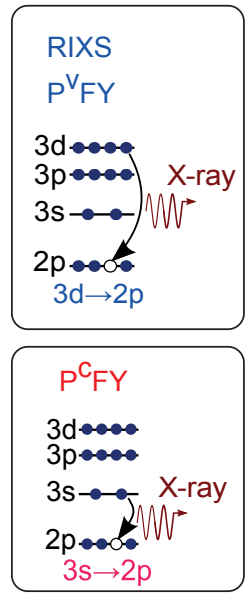

Figure 4: Experimental and calculated (RASSCF/RASPT2) spectra of the aqueous $\mathrm{Fe}^{2+}$ ion (an $\left[\mathrm{Fe}\left(\mathrm{H}_{2} \mathrm{O}\right)_{6}\right]^{2+}$ cluster is used in simulations, see inset). (a) Absorption spectra obtained in different modes $\stackrel{44}{4}$ true XAS (calculated - black), valence PFY (Equation (3)) with $\omega \in[660,720]$ eV denoted as $\mathrm{P}^{\mathrm{V}} \mathrm{FY}$ (experiment - blue, calculation - magenta) and core PFY $\omega \in[605,640] \mathrm{eV}$ denoted as $\mathrm{P}^{\mathrm{C}} \mathrm{FY}$ (experiment - red; calculation - green). (b) Respective RIXS spectra ${ }^{171}$ in energy loss $(\omega-\Omega$ in Equation (2) $)$ representation. The green and gray rectangles denote the energy range of the spin-allowed and formally spin-forbidden transitions, respectively. (c) Orbital scheme showing different radiative relaxation channels. (adapted from References 44, 171)

RIXS spectra of $\left[\mathrm{Fe}\left(\mathrm{H}_{2} \mathrm{O}\right)_{6}\right]^{2+}$ shown in Figure $4 \mathrm{~b}$ can be used to characterize the electronic structure in more detail $\frac{171}{17}$ For excitation across the $L_{3}$-edge up to its maximum, most prominent is the elastic Rayleigh feature at the energy loss of $0 \mathrm{eV}$. Inelastic peaks at about $-1.2 \mathrm{eV}$ energy loss are due to emission from the doubly occupied $t_{2 g}$ orbital and the respective shake-off satellites. With increasing excitation energy the intensity of inelastic peaks also increases as compared to the elastic ones. This evidences the fact that below $-1.2 \mathrm{eV}$ valence excited states are of triplet character. Essentially, the excitation occurs from the ground quintet state with spin $S=2$ to a spin-mixed core-excited state (see also Section 4.3 and Figure 6b). The features with energy losses 
from 0 to $-1.2 \mathrm{eV}$ correspond to $S \rightarrow$ mixed $\rightarrow S$ transitions. Due to the mixing in the intermediate state, the new formally spin-forbidden radiative channels $S \rightarrow$ mixed $\rightarrow S-1$ are opened. Since with increasing energy core-excitations are characterized by an increasing quintet-triplet mixing, the elastic peak decreases and spin-forbidden features grow in intensity. Different spin states of iron complexes have been also studied in Reference 288 .

The spectra shown in Figure $4 \mathrm{~b}$ are essentially due to local metal-centered transitions. RIXS features due to metal to ligand charge transfer are showing up around $-15 \mathrm{eV}$ energy loss, but have small intensity. Hence, the mixing between the iron and water orbitals at the $L$-edge is of minor importance, evidencing the predominant ionic character of the metal-ligand bond. Respective results obtained for probing the oxygen $K$-edge, yield rather similar spectra for $\left[\mathrm{Fe}\left(\mathrm{H}_{2} \mathrm{O}\right)_{6}\right]^{2+}$ and for pure water, thus supporting the weak mixing (ionic) picture $\frac{171}{11}$

Recording a true XAS in transmission mode is a difficult task for optically-thick condensed phase samples, such as solutions, even if microjets are utilized. Therefore, experimentally one usually obtains it in the PFY detection mode, Equation (3)! $\frac{26}{6}$ The natural question arising in this respect is how large are the distortions of the PFY relative to the true XAS and how they can be reduced $289 \sqrt[290]{290}$ Figure 4 a shows the two experimental PFY spectra resulting from $3 d \rightarrow 2 p$ $\left(\mathrm{P}^{\mathrm{V}} \mathrm{FY}\right)$ and $3 s \rightarrow 2 p\left(\mathrm{P}^{\mathrm{C}} \mathrm{FY}\right)$ dipole-allowed radiative relaxation of the initially created core hole, 44 see panel (c). The comparison of the calculated XAS and PFY spectra stemming from these two channels evidences that the core variant $\mathrm{P}^{\mathrm{C}} \mathrm{FY}$ should be a more reliable probe of XAS in contrast to $\mathrm{P}^{\mathrm{V}} \mathrm{FY}$. The reason for the distortions of the latter has been identified as being the opening

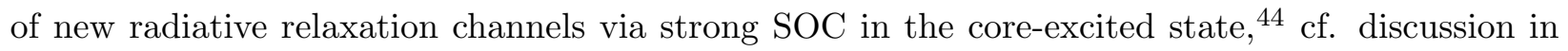
previous paragraph for RIXS.

The RIXS spectra record photon-in/photon-out events and thus are determined by dipole selection rules as is evident from the Kramers-Heisenberg Equation (2). In Reference 44, it has been demonstrated for $\left[\mathrm{Fe}\left(\mathrm{H}_{2} \mathrm{O}\right)_{6}\right]^{2+}$ that a back-to-back analysis of RIXS and photon-in/electron-out RPES, which is free from such selection rules, provides complementary information on the electronic states. In particular, comparing intensities, one can obtain information on the competition between radiative and non-radiative decay channels of the core-excitation.

\subsection{A new look at metal-ligand bonding}

XAS and RIXS spectroscopy applied to metal-ligand complexes can provide an atom-specific, chemical state selective, crystal field symmetry and orbital symmetry resolved description of the electronic 
structure $18,20,31,175$ In References $18,19,176$, it has been shown that this allows for scrutinizing traditional chemical concepts of metal ligand bonding. Within the valence bond structure theory, metal-ligand bonding is described by the so-called $\sigma$-donor $/ \pi$-acceptor mechanism. For example, the formation of the covalent $\mathrm{Fe}-\mathrm{CO}$ bonds in $\mathrm{Fe}(\mathrm{CO})_{5}$ is accompanied by charge donation from the $5 \sigma$ orbital of $\mathrm{CO}$ to the $\sigma 3 d$ orbital of the Fe and back donation to the $2 \pi^{*}$ orbital of $\mathrm{CO}$. In terms of the MOs of the metal-ligand complex, the donation/back donation is expressed by the mixing of the respective $\mathrm{Fe}$ and $\mathrm{CO}$ orbitals. The extent of this mixing can be addressed by identification of charge-transfer transitions in RIXS spectra.

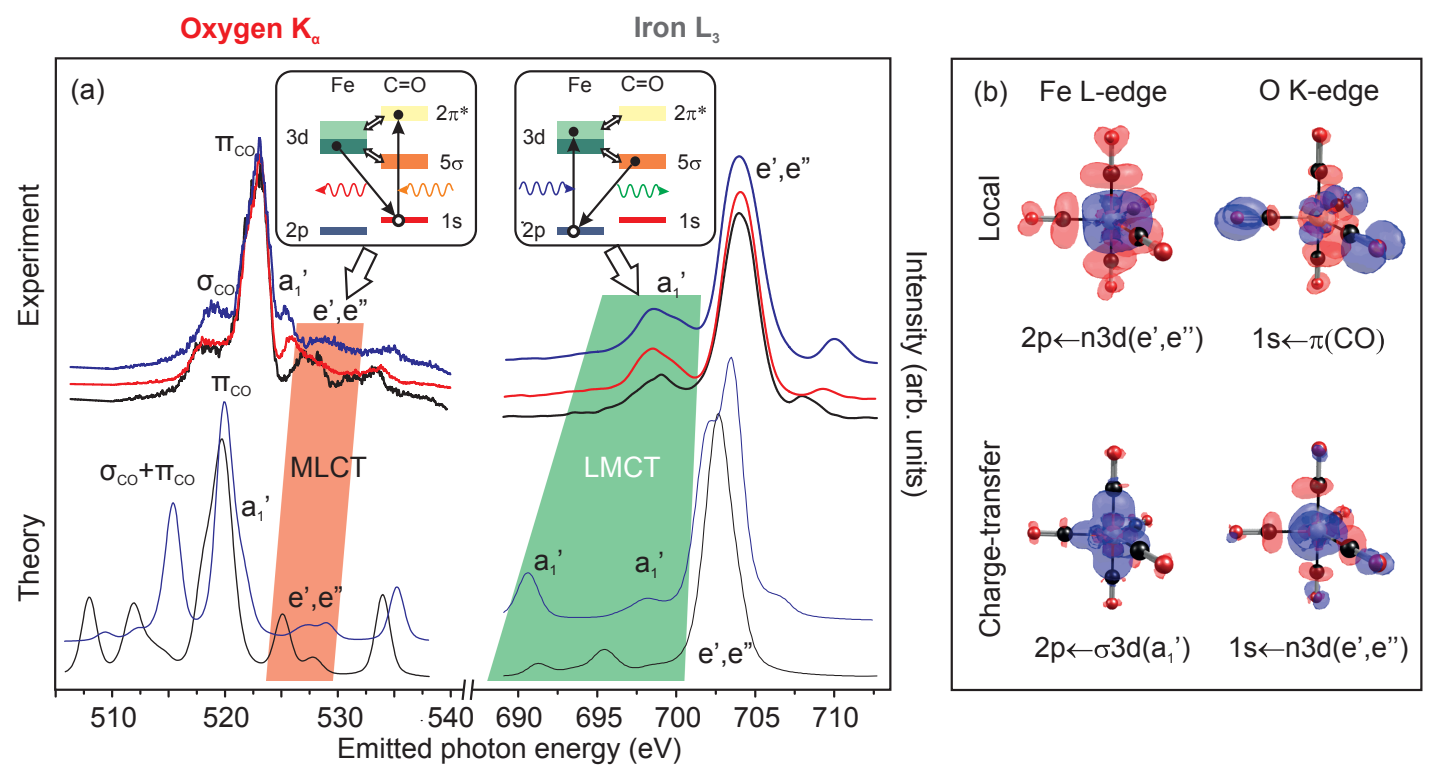

Figure 5: (a) Experimental and theoretical RIXS spectra of the $\mathrm{Fe}(\mathrm{CO})_{5}$ complex at iron $L$-edge and oxygen $K$-edge. The colored areas show the range of metal-to-ligand (MLCT) and ligand-tometal (LMCT) charge-transfer transitions which carry information about the strength of covalent bonding as explained in the respective insets. (b) Transition density difference plots which show the localization of the excited electron (red) and the electron which is refilling the core hole (blue) within the complex. (adapted from Reference 18)

For illustration, let us consider the RIXS amplitude for the Fe $L$-edge in the simplified picture considering only MOs. The dipole operator can be written as $\hat{d}=d_{2 p \rightarrow 3 d}|3 d\rangle\langle 2 p|+$ h.c. Suppose that the $3 d$ character of the absorbing state is given by the $\mathrm{MO}$ coefficient $C_{3 d}^{(\mathrm{abs})}$ of a virtual orbital, to which the core electron is excited, i.e. $\left|\Psi^{(\mathrm{abs})}\right\rangle=\hat{d}|2 p\rangle=d_{2 p \rightarrow 3 d} C_{3 d}^{(\mathrm{abs})}|3 d\rangle$. Thus, core excitation projects out all MO contributions apart from the $3 d$ due to locality and relatively strict dipole selec- 
tion rules. The $3 d$ character of the respective state for emission shall be given by the coefficient $C_{3 d}^{(\mathrm{em})}$ of the occupied orbital from which the core hole is refilled, i.e. $\left|\Psi^{(\mathrm{em})}\right\rangle=\hat{d}|3 d\rangle=d_{3 d \rightarrow 2 p} C_{3 d}^{(\mathrm{em})}|2 p\rangle$. Hence, the RIXS amplitude in Equation (2) becomes $\mathcal{R} \propto\left|d_{2 p \rightarrow 3 d}\right|^{4}\left|C_{3 d}^{(\mathrm{abs})}\right|^{2}\left|C_{3 d}^{(\mathrm{em})}\right|^{2}$. This suggests that comparing the intensities of different RIXS channels provides access to the character of the involved MOs. This has been demonstrated in References 18 and 19 for $\mathrm{Fe}(\mathrm{CO})_{5}$ and $\left[\mathrm{Fe}(\mathrm{CN})_{6}\right]^{4-}$, respectively. Needless to say that this reasoning applies to other absorption edges as well.

In Figure 5a, exemplary RIXS spectra for the Fe $L_{3}$-edge and the $\mathrm{O} K_{\alpha}$-edge of $\mathrm{Fe}(\mathrm{CO})_{5}$ are shown. In case of the Fe $L_{3}$-edge, there are, on the one hand side, local $d-d$ type radiative relaxation transitions of $n 3 d\left(e^{\prime}, e^{\prime \prime}\right)$ to $2 p$ character (see density difference plots in panel (b)). On the other hand side, inelastic peaks of $\sigma 3 d\left(a_{1}^{\prime}\right)$ to $2 p$ character are clearly discernible for different excitation energies. From the density difference plots (note especially the negative part shown in blue) in Figure 5b the ligand to metal charge-transfer character of these core-hole refill transitions becomes apparent. Based on the simple model outlined before the intensity ratio of these two types of transitions can be used to characterize the degree of orbital mixing. The same argument applied to the $\mathrm{O} K$-edge leads to the identification of the $n 3 d\left(e^{\prime}, e^{\prime \prime}\right)$ to 1 s transition as marker bands for the metal-to-ligand charge-transfer character. Taking both results together gives a means for quantifying the MO composition in terms of atomic orbitals or equivalently $\sigma$-donor $/ \pi$-acceptor character of the metal-ligand bonding. In order to establish the general nature of this argument, the $\left[\mathrm{Fe}(\mathrm{CN})_{6}\right]^{4-}$ complex has been investigated using RIXS spectroscopy ${ }^{[19}$ Comparing band intensities between $\left[\mathrm{Fe}(\mathrm{CN})_{6}\right]^{4-}$ and $\mathrm{Fe}(\mathrm{CO})_{5}$ it was verified that $\mathrm{CN}^{-}$is a stronger $\sigma$-donor but weaker $\pi$ acceptor than $\mathrm{CO}$, which is in accord with chemical intuition.

\subsection{Tracing catalytic activity}

Following the building principles of natural water-oxidation systems, manganese-based nanostructured catalysts have attracted considerable attention.291 293 X-ray spectroscopy is sensitive to the oxidation state of metal atoms. In Reference 21, this has been shown in a spectroscopic investigation of the redox evolution of manganese systems relevant for water photo-electro-oxidation. $294 \sqrt[295]{2}$ In the experiment, Mn precursors with different oxidation states have been doped into a nafion matrix, where all converted into $\mathrm{Mn}^{\mathrm{II}}$. These species can be oxidized upon application of a bias and reduced under visible light illumination. To follow this reaction, XAS as well as RIXS spectra have been calculated for the model compounds $\left[\mathrm{Mn}^{\mathrm{II}}\left(\mathrm{H}_{2} \mathrm{O}\right)_{6}\right]^{2+},\left[\mathrm{Mn}^{\mathrm{III}}\left(\mathrm{H}_{2} \mathrm{O}\right)_{6}\right]^{3+}$, and $\left[\mathrm{Mn}^{\mathrm{IV}} \mathrm{L}(\mathrm{OMe})_{3}\right]^{+}$ (cf. Figure 6) 21 Although the actual experimental systems are much more complex, the local 

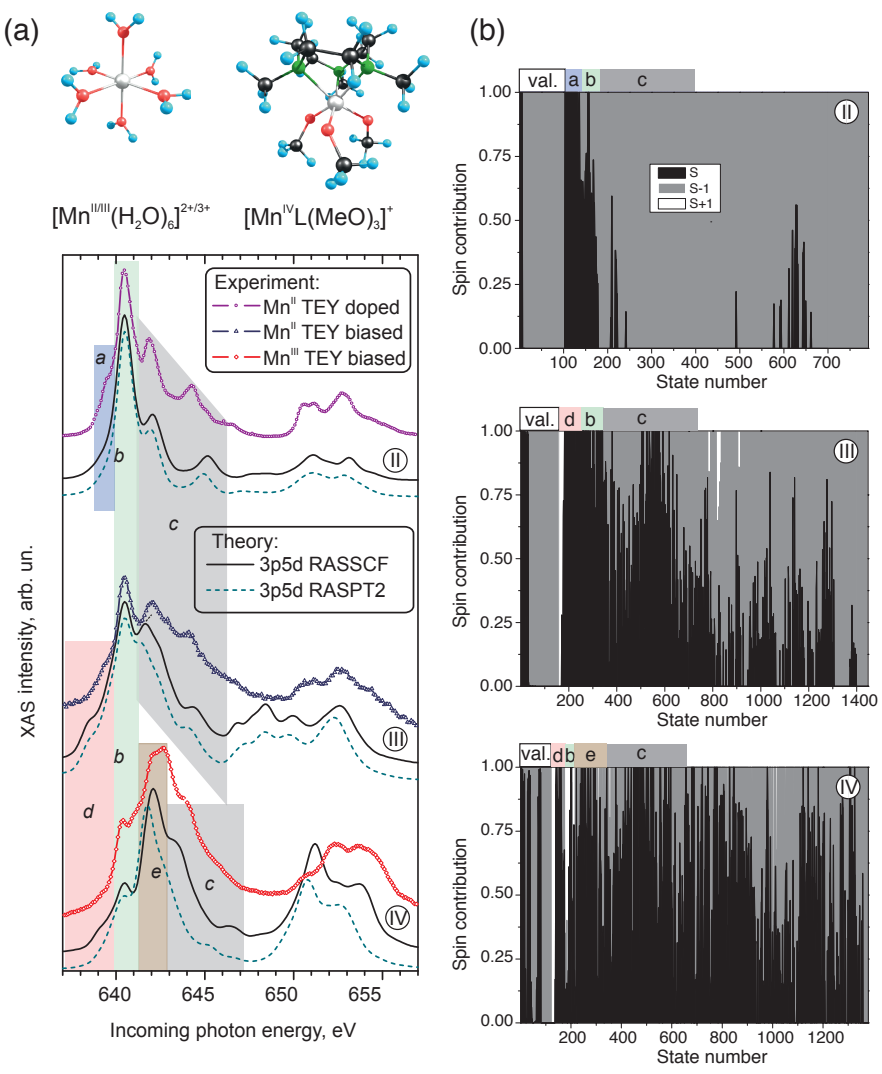

Figure 6: (a) Experimental and calculated (RASSCF/RASPT2) XAS spectra for Mn species in different oxidation states. (b) Contributions of states with $\Delta S=0, \pm 1$ to the spin-orbit coupled wave functions for the different species of panel (a). The labels (val,a-e) correspond to the bands marked in panel (a) where the widths of the shaded areas comprise the state numbers of panel (b) (adapted from Reference 21).

nature of the soft X-ray probe enables comparison with these model complexes. It turned out that for the present systems XAS is much more sensitive to the oxidation state than RIXS, hence only XAS spectra are shown in Figure 6. Comparison between experiment and simulation is hampered by the fact, that the former are done for mixtures of species having different oxidation states. Nevertheless, both spectra not only show strong similarities, but also a distinct dependence on the oxidation state.

The XAS spectra show the typical splitting between the $L_{2}$ and $L_{3}$ bands which is about 11 $\mathrm{eV}$, almost independent on the oxidation state. It follows that the $\mathrm{SOC}$ constant is about $7.3 \mathrm{eV}$. This large SOC constant leads to considerable coupling of core excited states of different spin, i.e. if $S$ denotes the spin of the electronic ground state, core-excited states are mixtures of $S-1, S$, 
and $S+1$ spin states. Any interpretation of the XAS spectra has to account for this fact. The decomposition of the spin-orbit coupled wave functions into $\Delta S=0, \pm 1$ contributions is given in Figure 6b for the different species. Whereas the valence excited states show little mixing, i.e. they are either of $\Delta S=0$ or $\Delta S=-1$ character, the situation is considerably more complex for coreexcited states, with the relative stability of spin-states being strongly affected by the core hole; for a more detailed discussion of the dependence on the actual species, see Reference 21.

The XAS spectra can be assigned as follows (in the following we discuss the $L_{3}$ band only, the $L_{2}$ band has a similar assignment, although the spin mixing is even stronger). (i) $\mathrm{Mn}^{\mathrm{II}}$ : Here, the low-energy shoulder (a) is of dominantly $2 p \rightarrow 3 d\left(t_{2 g}\right)$ character, comprising $\Delta S=0$ transitions. The main band (b) is shaped by intense $2 p \rightarrow 3 d\left(e_{g}\right)$ transitions with $\Delta S=0,-1$. The peaks denoted by (c) are due to mostly spin-forbidden $\Delta S=-1$ transitions of mixed $t_{2 g} / e_{g}$ character. (ii) $\mathrm{Mn}^{\mathrm{III}}$ : The spectrum is dominated by $2 p \rightarrow 3 d\left(e_{g}\right)$ transitions, but also contains shake-up $3 d\left(e_{g}\right) \rightarrow 3 d\left(t_{2 g}\right)$ transitions in region (b). Starting from the main peak (b) $\Delta S=0,-1$ spinmixing starts to play a role until the essentially spin-forbidden $\Delta S=-1$ region (c). (iii) $\mathrm{Mn}^{\mathrm{IV}}$ : the spectrum is similar to $\mathrm{Mn}^{\mathrm{III}}$, but also contains notable contribution from $\Delta S=+1$ transitions in regions (b,d,e). The influence of the PT2 correction to the RASSCF is also illustrated in Figure 6 . Apart from an overstabilization of the ground state which needs to be compensated by a constant shift of the spectrum, its shape hardly changes as can be seen in Figure 6 what is an evidence of mostly ionic metal-ligand bond character.

Analysis of inelastic RIXS spectra revealed that for all species most features are due to spinforbidden transitions, mediated by core-excited states ${ }^{21}$ similar to what has been discussed for iron complex in Section 4.1. Note that intricate spin mixing of the core-excited state gives rise to the exciting ultrafast spin-flip dynamics which can be triggered by XFEL and HHG X-ray pulses. $200,212,213$

\subsection{Aggregation and the challenge of multiple metal centers}

Straightforward extension of the RASSCF-based approach to the calculation of core-excited states of multi-center metal compounds would require active spaces that go beyond the capabilities of current hard- and software. Even if one would be able to perform such a calculation, the spectra would consist of hundreds of thousands of transitions, which would render any detailed interpretation of such spectra a challenging task. In order to address this problem, we have recently developed an approach which follows the logic of the so-called Frenkel exciton model $195 \mid 196$ The latter is used 
to describe the coupling of low-lying valence excitations in molecular aggregates and crystals, i.e. in situations where monomers assemble as a consequence of the van der Waals interaction. Since all what is needed is a localized excitation, the Frenkel exciton picture might apply to the present case of core-holes even if multiple metal centers in covalently bound complexes are considered.

The principal idea is to introduce metal center specific excitations which are in turn coupled by the Coulomb interaction. Thus, the Frenkel exciton Hamiltonian reads

$$
\begin{aligned}
\hat{H}^{\mathrm{FE}} & =\sum_{M} \sum_{A} E_{A_{M}}\left|A_{M}\right\rangle\left\langle A_{M}\right| \\
& +\frac{1}{2} \sum_{M N} \sum_{A, B, C, D} J_{M N}\left(A_{M} B_{N}, C_{N} D_{M}\right) \\
& \times\left|A_{M}\right\rangle\left\langle D_{M}|\otimes| B_{N}\right\rangle\left\langle C_{N}\right|
\end{aligned}
$$

where the states $A_{M}-D_{M}$ are eigenfunctions of the monomeric Hamiltonian, i.e. $H_{M}\left|A_{M}\right\rangle=$ $E_{A_{M}}\left|A_{M}\right\rangle$, and the Coulomb coupling can be expressed, e.g., in dipole approximation

$$
\begin{aligned}
J_{M N}\left(A_{M} B_{N}, C_{N} D_{M}\right) & \approx \frac{\mathbf{d}_{A_{M} D_{M}} \cdot \mathbf{d}_{B_{N} C_{N}}}{\left|\mathbf{X}_{M N}\right|^{3}} \\
& -3 \frac{\left(\mathbf{X}_{M N} \cdot \mathbf{d}_{A_{M} D_{M}}\right)\left(\mathbf{X}_{M N} \cdot \mathbf{d}_{B_{N} C_{N}}\right)}{\left|\mathbf{X}_{M N}\right|^{5}},
\end{aligned}
$$

where $\mathbf{d}_{A_{M} D_{M}}=\left\langle A_{M}|\hat{\mathbf{d}}| D_{M}\right\rangle$ is the transition dipole matrix element and the vector $\mathbf{X}_{M N}$ connects monomers $M$ and $N$.

The approach is illustrated in Figure $7 \mathrm{k}$ for the example of a dimer. The different monomeric state manifolds will be denoted as ground $\left|g_{M}\right\rangle$, valence-excited, $\left|v_{M}\right\rangle$, and core-excited, $\left|c_{M}\right\rangle$, states. Equation (20) contains couplings between all possible transitions in the dimer system. In the following, we will make use of the fact that the actual observables of interest, namely XAS and RIXS spectral amplitudes, are of first and second order with respect to the interaction with the external field. This suggests to employ either a one- or two-particle basis as shown in Figure 7 k. In the former, states of the type $\left|a_{1} g_{2}\right\rangle$ and $\left|g_{1} a_{2}\right\rangle(a=v, c)$ are incorporated, while the latter includes in addition states of type $\left|a_{1}, b_{2}\right\rangle(a, b=v, c)$ and is in principle exact for the dimer. Note that this effectively corresponds to a CISD-like treatment of the interaction between monomers in the composite system with X-ray specific preselection of configurations, while monomers are treated within the usual RASSCF procedure. In order to make this approach computationally feasible, a number of approximations can be introduced as detailed in Reference 196. Most important in this respect is the preselection of core-excited states to construct the monomeric basis functions, which is based on an energy window criterion for the excitation light pulse. 

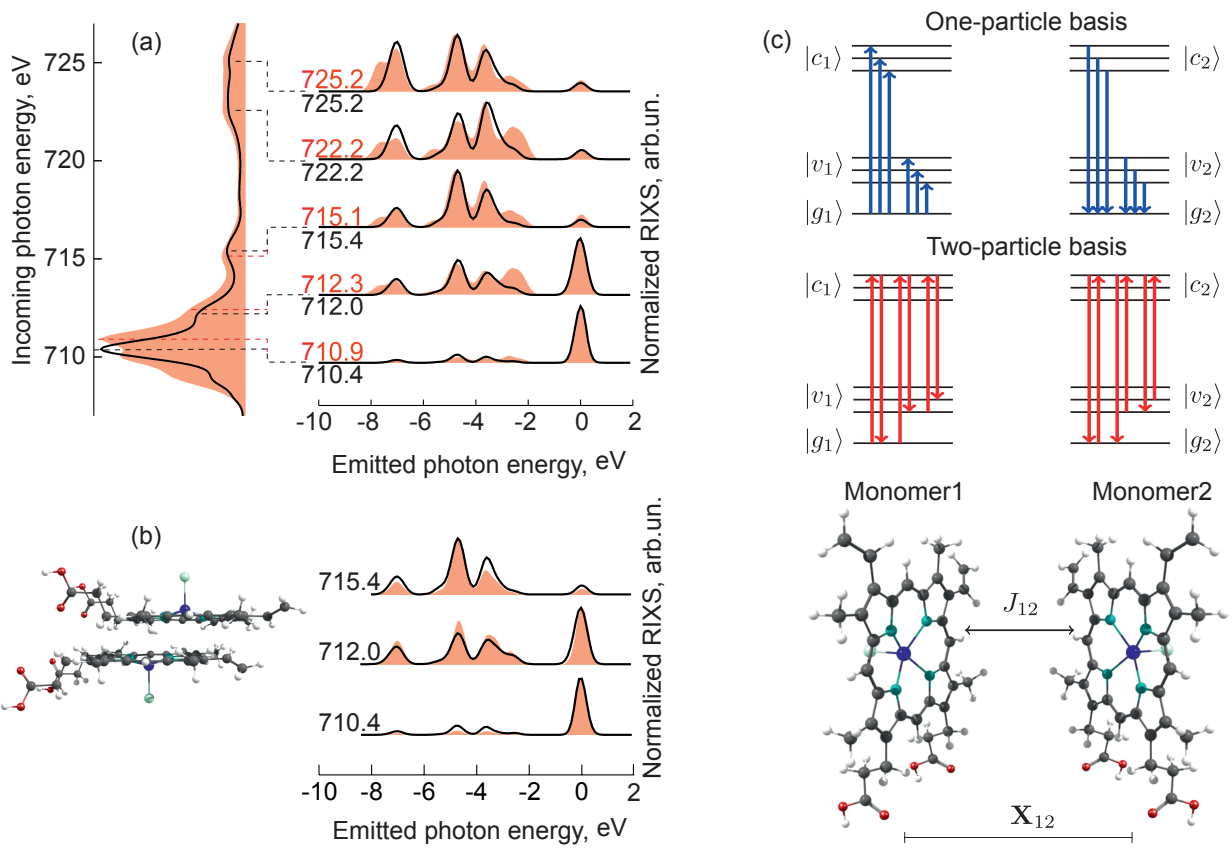

Figure 7: (a) Left: calculated normalized XAS for $[\text { heme B-Cl }]^{0}$ (black) and $\left[\text { heme } \mathrm{B}-\mathrm{H}_{2} \mathrm{O}\right]^{+}$(red filled curves). Right: normalized RIXS spectra for selected excitation energies. (b) Calculated RIXS spectra for the hemin dimer (red filled curves) with the $-\mathrm{COOH}$ groups pointing in the same direction. The monomer spectrum is also shown $(\times 2$, black lines $)$. Note that monomer and dimer XAS are indistinguishable on this scale. (c) Different choices of excitonic bases and the corresponding transitions included in the dimer coupling. For the one-particle basis, only transitions from or to the ground state have been included. For the two-particle basis, de-excitations from an arbitrary core-excited state to any other state are allowed. (adapted from Reference 196)

In a proof-of-concept study, this approach has been applied to investigate the signatures of aggregation of hemin in solution. ${ }^{196}$ Hemin shows a solvent dependent aggregation behavior, staying monomeric in polar solvents like ethanol or DMSO, while it forms dimers in water solution. Experimentally, the effect of aggregation was addressed by means of soft X-ray Fe $L$-edge XAS as well as by NXES and RIXS on the example of DMSO and aqueous solutions. $296 \mid 297$ The general shape of the spectra for both cases was quite similar and the pronounced difference in broadenings for RIXS as well as a $1.3 \mathrm{eV}$ energy shift in off-resonant NXES was attributed to aggregation. However, judging the effect of aggregation one should account for the labile equilibrium between different species in solution that also could be a source of spectral changes.

In Figure $7 \mathrm{a}$, XAS as well as selected RIXS spectra are shown for the bare hemin molecule 
$\left([\text { Heme } \mathrm{B}-\mathrm{Cl}]^{0}\right)$ as well as for the case where the axial $\mathrm{Cl}^{-}$ligand has been exchanged by a water molecule $\left(\left[\text { Heme } \mathrm{B}-\mathrm{H}_{2} \mathrm{O}\right]^{+}\right)$. The XAS spectrum shows a distinct sensitivity of the $L_{3}$ band to the type of ligand. In the present case, changing the ligand from $\mathrm{Cl}^{-}$to $\mathrm{H}_{2} \mathrm{O}$ causes a splitting of the $L_{3}$ peak in two components at $710.0 \mathrm{eV}$ and $710.9 \mathrm{eV}$. While the main peak is due to $2 p \rightarrow d_{x^{2}-y^{2}}$ and $d_{z^{2}}$ excitations, the splitting results from the energetic lowering of $2 p \rightarrow d_{z^{2}}$ with respect to $2 p \rightarrow d_{x^{2}-y^{2}}$ transitions.

The RIXS spectra of the monomer show a trend similar to the one discussed in Section 4.1. For excitation energies above $720 \mathrm{eV}$, the core-excited states are mostly of quartet type. Here, the most intense emission goes to valence-excited states with high quartet contributions. The RIXS spectra for both species differ mostly in the inelastic peaks, whereas the elastic peak has a comparable intensity in both spectra.

The comparison of $[\mathrm{Heme} \mathrm{B}-\mathrm{Cl}]^{0}$ dimer and monomer spectra is shown in Figure $7 \mathrm{~b}$. The XAS spectra are essentially identical, i.e. there is almost no effect due to dimerization. This can be attributed to the intensities of the metal $2 p \rightarrow 3 d$ and $3 d \rightarrow 3 d$ transitions relevant for $L$-edge X-ray spectra that are lower (due to smaller radial overlap and dipole selection rules) than those of the $\pi \rightarrow \pi^{*}$ and $n \rightarrow \pi^{*}$ transitions usually discussed in the case of aggregates of organic dyes. However, distinct fingerprints of dimerization can be seen in RIXS, in both the inelastic and elastic features. Overall, the magnitude of the effect of dimerization is comparable to the differences observed upon ligand exchange in the monomer. Hence, the unequivocal identification of spectral features due to solvent dependent dimerization is not a straightforward task.

\section{CONCLUSIONS}

There is a plethora of methods which have been developed to calculate core-excited states for prediction of various X-ray spectroscopic signals. For TM compounds beyond the $K$-edge, multireference approaches provide the methods of choice as they can deal with the intricate multiplet structure shaped by static and dynamic correlations and substantial SOC. However, limitations are due to the methods' complexity and associated computational effort. Here, active space approaches and in particular RASSCF are playing a prominent role as they are, in principle, exact and systematically improvable by increasing the size of the active space. In practice, however, methods like RASSCF cannot be considered as a "black box" due to the strong influence of the choice of the AS. Recent progress in the field of Density Matrix Renormalization Group (DMRG) and automated 
AS selection certainly mitigates this problem. ${ }^{298}$ For polymetallic complexes, merging established ideas of electronic structure theory with models developed in other areas such as the Frenkel exciton Hamiltonian could broaden the range of systems for theoretical X-ray spectroscopic studies.

To date, there is a considerable record of theoretical and experimental applications of frequency-

domain X-ray techniques to study TM complexes in various environments. With the emerging explicit time-domain techniques, either using XFEL or HHG facilities, nonlinear spectroscopy in the X-ray domain will play a role for unraveling electron dynamics, comparable to that of UV/Vis in case of (non-)Born-Oppenheimer electron-nuclear dynamics. ${ }^{241}$

\section{ACKNOWLEDGEMENTS}

We would like to acknowledge the financial support from the Deutsche Forschungsgemeinschaft via grant No. BO 4915/1-1 (S.I.B.) and Ku952/10-1 (O.K.) and the Deanship of Scientific Research (DSR), King Abdulaziz University, Jeddah, (Grant No. D-003-435).

\section{References}

[1] Chergui M. Time-Resolved X-Ray Spectroscopies of Chemical Systems: New Perspectives. Struct Dyn. 2016;3:031001.

[2] Young L, Ueda K, Gühr M, Bucksbaum PH, Simon M, Mukamel S, et al. Roadmap of Ultrafast X-Ray Atomic and Molecular Physics. J Phys B. 2018;51:032003.

[3] Stöhr J. NEXAFS Spectroscopy. Berlin, New York: Springer-Verlag; 1992.

[4] de Groot F, Kotani A. Core Level Spectroscopy of Solids. Boca Raton: CRC Press; 2008.

[5] Milne CJ, Penfold TJ, Chergui M. Recent Experimental and Theoretical Developments in Time-Resolved X-Ray Spectroscopies. Coord Chem Rev. 2014;277-278:44-68.

[6] Chergui M, Collet E. Photoinduced Structural Dynamics of Molecular Systems Mapped by Time-Resolved X-Ray Methods. Chem Rev. 2017;117:11025-11065.

[7] Rehr JJ, Ankudinov AL. Progress in the Theory and Interpretation of XANES. Coord Chem Rev. 2005;249:131-140. 
[8] Leng X, Jin F, Wei M, Ma Y. GW Method and Bethe-Salpeter Equation for Calculating Electronic Excitations: GW Method and Bethe-Salpeter Equation. Wiley Interdiscip Rev: Comput Mol Sci. 2016;6:532-550.

[9] Schnohr CS, Ridgway MC. Introduction to X-Ray Absorption Spectroscopy. In: Schnohr CS, Ridgway MC, editors. X-Ray Absorption Spectroscopy of Semiconductors. vol. 190. Berlin, Heidelberg: Springer Berlin Heidelberg; 2015. p. 1-26.

[10] Besley NA, Asmuruf FA. Time-Dependent Density Functional Theory Calculations of the Spectroscopy of Core Electrons. Phys Chem Chem Phys. 2010;12:12024.

[11] DeBeer S, Neese F. X-Ray Spectroscopy. In: Comprehensive Inorganic Chemistry II. Elsevier; 2013. p. $427-439$.

[12] Norman P, Dreuw A. Simulating X-Ray Spectroscopies and Calculating Core-Excited States of Molecules. Chem Rev. 2018;118:7208-7248.

[13] Hämäläinen K, Siddons DP, Hastings JB, Berman LE. Elimination of the Inner-Shell Lifetime Broadening in x-Ray-Absorption Spectroscopy. Phys Rev Lett. 1991;67:2850-2853.

[14] Piancastelli MN, Goldsztejn G, Marchenko T, Guillemin R, Kushawaha RK, Journel L, et al. Core-Hole-Clock Spectroscopies in the Tender x-Ray Domain. J Phys B. 2014;47:124031.

[15] Pollock CJ, Grubel K, Holland PL, DeBeer S. Experimentally Quantifying Small-Molecule Bond Activation Using Valence-to-Core X-Ray Emission Spectroscopy. J Am Chem Soc. 2013;135:11803-11808.

[16] Soldatov MA, Martini A, Bugaev AL, Pankin I, Medvedev PV, Guda AA, et al. The Insights from X-Ray Absorption Spectroscopy into the Local Atomic Structure and Chemical Bonding of Metal-Organic Frameworks. Polyhedron. 2018;155:232-253.

[17] Mastelaro V, Zanotto E. X-Ray Absorption Fine Structure (XAFS) Studies of Oxide Glasses-A 45-Year Overview. Materials. 2018;11:204.

[18] Suljoti E, Garcia-Diez R, Bokarev SI, Lange KM, Schoch R, Dierker B, et al. Direct Observation of Molecular Orbital Mixing in a Solvated Organometallic Complex. Angew Chem Int Ed. 2013;52:9841-9844. 
[19] Engel N, Bokarev SI, Suljoti E, Garcia-Diez R, Lange KM, Atak K, et al. Chemical Bonding in Aqueous Ferrocyanide: Experimental and Theoretical X-Ray Spectroscopic Study. J Phys Chem B. 2014;118:1555-1563.

[20] Kunnus K, Zhang W, Delcey MG, Pinjari RV, Miedema PS, Schreck S, et al. Viewing the Valence Electronic Structure of Ferric and Ferrous Hexacyanide in Solution from the Fe and Cyanide Perspectives. J Phys Chem B. 2016;120:7182-7194.

[21] Bokarev SI, Khan M, Abdel-Latif MK, Xiao J, Hilal R, Aziz SG, et al. Unraveling the Electronic Structure of Photocatalytic Manganese Complexes by L-Edge X-Ray Spectroscopy. J Phys Chem C. 2015;119:19192-19200.

[22] Wernet P, Kunnus K, Josefsson I, Rajkovic I, Quevedo W, Beye M, et al. Orbital-Specific Mapping of the Ligand Exchange Dynamics of Fe(CO) $)_{5}$ in Solution. Nature. 2015;520:78-81.

[23] Vankó G, Bordage A, Pápai M, Haldrup K, Glatzel P, March AM, et al. Detailed Characterization of a Nanosecond-Lived Excited State: X-Ray and Theoretical Investigation of the Quintet State in Photoexcited $\left[\mathrm{Fe}(\text { Terpy })_{2}\right]^{2+}$. J Phys Chem C. 2015;119:5888-5902.

[24] Kubin M, Guo M, Kroll T, Löchel H, Källman E, Baker ML, et al. Probing the Oxidation State of Transition Metal Complexes: A Case Study on How Charge and Spin Densities Determine Mn L-Edge X-Ray Absorption Energies. Chem Sci. 2018;9:6813-6829.

[25] Hüfner S. Photoelectron Spectroscopy: Principles and Applications. Berlin, Heidelberg: Springer; 2003.

[26] Sham TK. Photon-In Photon-Out Spectroscopic Techniques for Materials Analysis: Some Recent Developments. In: Fan C, Zhao Z, editors. Synchrotron Radiation in Materials Science. Weinheim, Germany: Wiley-VCH Verlag GmbH \& Co. KGaA; 2018. p. 123-136.

[27] Liekhus-Schmaltz CE, Tenney I, Osipov T, Sanchez-Gonzalez A, Berrah N, Boll R, et al. Ultrafast Isomerization Initiated by X-Ray Core Ionization. Nat Commun. 2015;6:8199.

[28] Pertot Y, Schmidt C, Matthews M, Chauvet A, Huppert M, Svoboda V, et al. Time-Resolved x-Ray Absorption Spectroscopy with a Water Window High-Harmonic Source. Science. 2017;355:264-267. 
[29] Hong K, Cho H, Schoenlein RW, Kim TK, Huse N. Element-Specific Characterization of Transient Electronic Structure of Solvated Fe(II) Complexes with Time-Resolved Soft X-Ray Absorption Spectroscopy. Acc Chem Res. 2015;48:2957-2966.

[30] Wörner HJ, Arrell CA, Banerji N, Cannizzo A, Chergui M, Das AK, et al. Charge Migration and Charge Transfer in Molecular Systems. Struct Dyn. 2017;4:061508.

[31] Jay RM, Norell J, Eckert S, Hantschmann M, Beye M, Kennedy B, et al. Disentangling Transient Charge Density and Metal-Ligand Covalency in Photoexcited Ferricyanide with Femtosecond Resonant Inelastic Soft X-Ray Scattering. J Phys Chem Lett. 2018;9:35383543.

[32] Newton M. Time Resolved Operando X-Ray Techniques in Catalysis, a Case Study: CO Oxidation by $\mathrm{O}_{2}$ over Pt Surfaces and Alumina Supported Pt Catalysts. Catalysts. 2017;7:58.

[33] Beye M, Öberg H, Xin H, Dakovski GL, Dell'Angela M, Föhlisch A, et al. Chemical Bond Activation Observed with an X-Ray Laser. J Phys Chem Lett. 2016;7:3647-3651.

[34] Rimmerman D, Leshchev D, Hsu DJ, Hong J, Kosheleva I, Chen LX. Direct Observation of Insulin Association Dynamics with Time-Resolved X-Ray Scattering. J Phys Chem Lett. 2017;8:4413-4418.

[35] Brauße F, Goldsztejn G, Amini K, Boll R, Bari S, Bomme C, et al. Time-Resolved InnerShell Photoelectron Spectroscopy: From a Bound Molecule to an Isolated Atom. Phys Rev A. 2018;97:043429.

[36] Squibb RJ, Sapunar M, Ponzi A, Richter R, Kivimäki A, Plekan O, et al. Acetylacetone Photodynamics at a Seeded Free-Electron Laser. Nat Commun. 2018;9:63.

[37] Seidler GT, Mortensen DR, Remesnik AJ, Pacold JI, Ball NA, Barry N, et al. A LaboratoryBased Hard x-Ray Monochromator for High-Resolution x-Ray Emission Spectroscopy and x-Ray Absorption near Edge Structure Measurements. Rev Sci Instrum. 2014;85:113906.

[38] Mantouvalou I, Witte K, Grötzsch D, Neitzel M, Günther S, Baumann J, et al. High Average Power, Highly Brilliant Laser-Produced Plasma Source for Soft X-Ray Spectroscopy. Rev Sci Instrum. 2015;86:035116. 
[39] Winter B, Faubel M. Photoemission from Liquid Aqueous Solutions. Chem Rev. 2006;106:1176-1211.

[40] Smith JW, Saykally RJ. Soft X-Ray Absorption Spectroscopy of Liquids and Solutions. Chem Rev. 2017;117:13909-13934.

[41] Kotani A, Shin S. Resonant Inelastic X-Ray Scattering Spectra for Electrons in Solids. Rev Mod Phys. 2001;73:203.

[42] Gel'mukhanov F, Ågren H. Resonant X-Ray Raman Scattering. Phys Rep. 1999;312:87-330.

[43] Averbukh V, Demekhin PV, Kolorenč P, Scheit S, Stoychev SD, Kuleff AI, et al. Interatomic Electronic Decay Processes in Singly and Multiply Ionized Clusters. J Electron Spectrosc Relat Phenom. 2011;183:36-47.

[44] Golnak R, Bokarev SI, Seidel R, Xiao J, Grell G, Atak K, et al. Joint Analysis of Radiative and Non-Radiative Electronic Relaxation Upon X-Ray Irradiation of Transition Metal Aqueous Solutions. Sci Rep. 2016;6:24659.

[45] Ballentine LE. Quantum Mechanics: A Modern Development. Singapore: World Scientific; 2010.

[46] May V, Kühn O. Charge and Energy Transfer Dynamics in Molecular Systems. Weinheim: Wiley-VCH; 2011.

[47] Bernadotte S, Atkins AJ, Jacob CR. Origin-Independent Calculation of Quadrupole Intensities in X-Ray Spectroscopy. J Chem Phys. 2012;137:204106.

[48] Mukamel S. Principles of Nonlinear Optical Spectroscopy. New York: Oxford Univ. Press; 1999.

[49] Fadley CS. X-Ray Photoelectron Spectroscopy: Progress and Perspectives. J Electron Spectrosc Relat Phenom. 2010;178-179:2-32.

[50] Fadley CS, Nemšák S. Some Future Perspectives in Soft- and Hard- X-Ray Photoemission. J Electron Spectrosc Relat Phenom. 2014;195:409-422.

[51] Norell J, Grell G, Kühn O, Odelius M, Bokarev SI. Photoelectron Shake-Ups as a Probe of Molecular Symmetry: 4d XPS Analysis of $\mathrm{I}_{3}^{-}$in Solution. Phys Chem Chem Phys. 2018;20:19916-19921. 
[52] Cortes M, Martin F. Multichannel Close-Coupling Method with L 2 Integrable Bases. J Phys B. $1994 ; 27: 5741-5760$.

[53] Grell G, Bokarev SI, Winter B, Seidel R, Aziz EF, Aziz SG, et al. Multi-Reference Approach to the Calculation of Photoelectron Spectra Including Spin-Orbit Coupling. J Chem Phys. $2015 ; 143: 074104$.

[54] Oana CM, Krylov AI. Dyson Orbitals for Ionization from the Ground and Electronically Excited States within Equation-of-Motion Coupled-Cluster Formalism: Theory, Implementation, and Examples. J Chem Phys. 2007;127:234106.

[55] Möhle T, Bokareva OS, Grell G, Kühn O, Bokarev SI. Tuned Range-Separated Density Functional Theory and Dyson Orbital Formalism for Photoelectron Spectra. J Chem Theor Comput. 2018;14:5870-5880.

[56] Moguilevski A, Wilke M, Grell G, Bokarev SI, Aziz SG, Engel N, et al. Ultrafast Spin Crossover in $\left[\mathrm{Fe}^{\mathrm{II}}(\mathrm{bpy})_{3}\right]^{2+}$ : Revealing Two Competing Mechanisms by Extreme Ultraviolet Photoemission Spectroscopy. ChemPhysChem. 2017;18:465-469.

[57] Raheem AA, Wilke M, Borgwardt M, Engel N, Bokarev SI, Grell G, et al. Ultrafast Kinetics of Linkage Isomerism in $\mathrm{Na}_{2}\left[\mathrm{Fe}(\mathrm{CN})_{5} \mathrm{NO}\right]$ Aqueous Solution Revealed by Time-Resolved Photoelectron Spectroscopy. Struct Dyn. 2017;4:044031.

[58] Engel N, Bokarev SI, Moguilevski A, Raheem AA, Al-Obaidi R, Möhle T, et al. Light-Induced Relaxation Dynamics of the Ferricyanide Ion Revisited by Ultrafast XUV Photoelectron Spectroscopy. Phys Chem Chem Phys. 2017;19:14248-14255.

[59] Åberg T. Theory of X-Ray Satellites. Phys Rev. 1967;156:35-41.

[60] Neese F, Petrenko T, Ganyushin D, Olbrich G. Advanced Aspects of Ab Initio Theoretical Optical Spectroscopy of Transition Metal Complexes: Multiplets, Spin-Orbit Coupling and Resonance Raman Intensities. Coord Chem Rev. 2007;251:288-327.

[61] Karsten S, Ivanov SD, Aziz SG, Bokarev SI, Kühn O. Nuclear Dynamical Correlation Effects in X-Ray Spectroscopy from a Theoretical Time-Domain Perspective. J Phys Chem Lett. 2017;8:992-996. 
[62] Karsten S, Bokarev SI, Aziz SG, Ivanov SD, Kühn O. A Time-Correlation Function Approach to Nuclear Dynamical Effects in X-Ray Spectroscopy. J Chem Phys. 2017;146:224203.

[63] Karsten S, Ivanov SD, Bokarev SI, Kühn O. Quasi-Classical Approaches to Vibronic Spectra Revisited. J Chem Phys. 2018;148:102337.

[64] Karsten S, Ivanov SD, Bokarev SI, Kühn O. Simulating Vibronic Spectra via Matsubara-like Dynamics: Coping with the Sign Problem. J Chem Phys. 2018;149:194103.

[65] Kümmel S, Kronik L. Orbital-Dependent Density Functionals: Theory and Applications. Rev Mod Phys. 2008;80:3-60.

[66] Hedin L, Johansson A. Polarization Corrections to Core Levels. J Phys B. 1969;2:1336-1346.

[67] Smolentsev G, Soldatov AV, Messinger J, Merz K, Weyhermüller T, Bergmann U, et al. X-Ray Emission Spectroscopy To Study Ligand Valence Orbitals in Mn Coordination Complexes. J Am Chem Soc. 2009;131:13161-13167.

[68] Jolly WL, Hendrickson DN. Thermodynamic Interpretation of Chemical Shifts in CoreElectron Binding Energies. J Am Chem Soc. 1970;92:1863-1871.

[69] Ågren H, Medina-Llanos C, Mikkelsen KV, Jensen HJA. On the Validity of the Equivalent Core Approximation in Born-Haber Analyses of Liquids and Solutions. Chem Phys Lett. $1988 ; 153: 322-327$.

[70] Bagus PS. Self-Consistent-Field Wave Functions for Hole States of Some Ne-Like and Ar-Like Ions. Phys Rev. 1965;139:A619-A634.

[71] Evangelista FA, Shushkov P, Tully JC. Orthogonality Constrained Density Functional Theory for Electronic Excited States. J Phys Chem A. 2013;117:7378-7392.

[72] Derricotte WD, Evangelista FA. Simulation of X-Ray Absorption Spectra with Orthogonality Constrained Density Functional Theory. Phys Chem Chem Phys. 2015;17:14360-14374.

[73] Hsu Hl, Davidson ER, Pitzer RM. An SCF Method for Hole States. J Chem Phys. 1976;65:609-613.

[74] Gilbert ATB, Besley NA, Gill PMW. Self-Consistent Field Calculations of Excited States Using the Maximum Overlap Method (MOM). J Phys Chem A. 2008;112:13164-13171. 
[75] Besley NA, Gilbert ATB, Gill PMW. Self-Consistent-Field Calculations of Core Excited States. J Chem Phys. 2009;130:124308.

[76] Jensen HJA, Jørgensen P, Ågren H. Efficient Optimization of Large Scale MCSCF Wave Functions with a Restricted Step Algorithm. J Chem Phys. 1987;87:451-466.

[77] Naves de Brito A, Correia N, Svensson S, Ågren H. A Theoretical Study of X-ray Photoelectron Spectra of Model Molecules for Polymethylmethacrylate. J Chem Phys. 1991;95:29652974 .

[78] Chong DP. Accurate Calculation of Core-Electron Binding Energies by the DensityFunctional Method. Chem Phys Lett. 1995;232:486-490.

[79] Prendergast D, Galli G. X-Ray Absorption Spectra of Water from First Principles Calculations. Phys Rev Lett. 2006;96:215502.

[80] Triguero L, Pettersson LGM, Ågren H. Calculations of Near-Edge x-Ray-Absorption Spectra of Gas-Phase and Chemisorbed Molecules by Means of Density-Functional and TransitionPotential Theory. Phys Rev B. 1998;58:8097-8110.

[81] Leetmaa M, Ljungberg MP, Lyubartsev A, Nilsson A, Pettersson LGM. Theoretical Approximations to X-Ray Absorption Spectroscopy of Liquid Water and Ice. J Electron Spectrosc Relat Phenom. 2010;177:135-157.

[82] Theophilou AK. The Energy Density Functional Formalism for Excited States. J Phys C. 1979;12:5419-5430.

[83] Filatov M. Ensemble DFT Approach to Excited States of Strongly Correlated Molecular Systems. In: Ferré N, Filatov M, Huix-Rotllant M, editors. Density-Functional Methods for Excited States. vol. 368. Cham: Springer International Publishing; 2015. p. 97-124.

[84] Perdew JP, Parr RG, Levy M, Balduz JL. Density-Functional Theory for Fractional Particle Number: Derivative Discontinuities of the Energy. Phys Rev Lett. 1982;49:1691-1694.

[85] Mori-Sánchez P, Cohen AJ, Yang W. Localization and Delocalization Errors in Density Functional Theory and Implications for Band-Gap Prediction. Phys Rev Lett. 2008;100:146401.

[86] Williams AR, deGroot RA, Sommers CB. Generalization of Slater's Transition State Concept. J Chem Phys. 1975;63:628-631. 
[87] Ehlert C, Klamroth T. The Quest for Best Suited References for Configuration Interaction Singles Calculations of Core Excited States. J Comput Chem. 2017;38:116-126.

[88] Koopmans T. Über Die Zuordnung von Wellenfunktionen Und Eigenwerten Zu Den Einzelnen Elektronen Eines Atoms. Physica. 1934;1:104-113.

[89] Almbladh CO, von Barth U. Exact Results for the Charge and Spin Densities, ExchangeCorrelation Potentials, and Density-Functional Eigenvalues. Phys Rev B. 1985;31:3231-3244.

[90] Chong DP, Gritsenko OV, Baerends EJ. Interpretation of the Kohn-Sham Orbital Energies as Approximate Vertical Ionization Potentials. J Chem Phys. 2002;116:1760-1772.

[91] Lee N, Petrenko T, Bergmann U, Neese F, DeBeer S. Probing Valence Orbital Composition with Iron K $\beta$ X-Ray Emission Spectroscopy. J Am Chem Soc. 2010;132:9715-9727.

[92] Lassalle-Kaiser B, Boron TT, Krewald V, Kern J, Beckwith MA, Delgado-Jaime MU, et al. Experimental and Computational X-Ray Emission Spectroscopy as a Direct Probe of Protonation States in Oxo-Bridged $\mathrm{Mn}^{\mathrm{IV}}$ Dimers Relevant to Redox-Active Metalloproteins. Inorg Chem. 2013;52:12915-12922.

[93] Liang Y, Vinson J, Pemmaraju S, Drisdell WS, Shirley EL, Prendergast D. Accurate X-Ray Spectral Predictions: An Advanced Self-Consistent-Field Approach Inspired by Many-Body Perturbation Theory. Phys Rev Lett. 2017;118:096402.

[94] Triguero L, Plashkevych O, Pettersson LGM, Ågren H. Separate State vs. Transition State Kohn-Sham Calculations of X-Ray Photoelectron Binding Energies and Chemical Shifts. J Electron Spectrosc Relat Phenom. 1999;104:195-207.

[95] Takahashi O, Pettersson LGM. Functional Dependence of Core-Excitation Energies. J Chem Phys. 2004;121:10339-10345.

[96] Ferré N, Assfeld X. Application of the Local Self-Consistent-Field Method to Core-Ionized and Core-Excited Molecules, Polymers, and Proteins: True Orthogonality between Ground and Excited States. J Chem Phys. 2002;117:4119-4125.

[97] Loos PF, Assfeld X. Core-Ionized and Core-Excited States of Macromolecules. Int J Quant Chem. 2007;107:2243-2252. 
[98] Hetényi B, De Angelis F, Giannozzi P, Car R. Calculation of Near-Edge x-Ray-Absorption Fine Structure at Finite Temperatures: Spectral Signatures of Hydrogen Bond Breaking in Liquid Water. J Chem Phys. 2004;120:8632-8637.

[99] Ågren H, Svensson S, Whalgren UI. SCF and Limited CI Calculations for Assignment of the Auger Spectrum and of the Satellites in the Soft X-Ray Spectrum of $\mathrm{H}_{2} \mathrm{O}$. Chem Phys Lett. 1975;35:336-344.

[100] Svensson S, Ågren H, Wahlgren UI. SCF and Limited CI Calculations on the 1s Shake-up Spectrum of $\mathrm{H}_{2} \mathrm{O}$. Chem Phys Lett. 1976;38:1-8.

[101] Shavitt I. The Method of Configuration Interaction. In: Schaefer HF, editor. Methods of Electronic Structure Theory. Boston, MA: Springer US; 1977. p. 189-275.

[102] Sherrill CD, Schaefer HF. The Configuration Interaction Method: Advances in Highly Correlated Approaches. In: Adv. Quantum Chem.. vol. 34. Elsevier; 1999. p. 143-269.

[103] Davidson ER. The Iterative Calculation of a Few of the Lowest Eigenvalues and Corresponding Eigenvectors of Large Real-Symmetric Matrices. J Comput Phys. 1975;17:87-94.

[104] Cederbaum LS, Domcke W, Schirmer J. Many-Body Theory of Core Holes. Phys Rev A. 1980;22:206-222.

[105] Barth A, Cederbaum LS. Many-Body Theory of Core-Valence Excitations. Phys Rev A. 1981;23:1038-1061.

[106] Schirmer J, Braunstein M, McKoy V. Molecular K-Shell Photoionization Cross Sections in the Relaxed-Core Hartree-Fock Approximation. Phys Rev A. 1990;41:283-300.

[107] Stener M, Fronzoni G, de Simone M. Time Dependent Density Functional Theory of Core Electrons Excitations. Chem Phys Lett. 2003;373:115-123.

[108] Ray K, DeBeer George S, Solomon EI, Wieghardt K, Neese F. Description of the Ground-State Covalencies of the Bis(Dithiolato) Transition-Metal Complexes from X-Ray Absorption Spectroscopy and Time-Dependent Density-Functional Calculations. Chem Eur J. 2007;13:27832797.

[109] Barth A, Schirmer J. Theoretical Core-Level Excitation Spectra of $\mathrm{N}_{2}$ and CO by a New Polarisation Propagator Method. J Phys B. 1985;18:867-885. 
[110] Trofimov AB, Moskovskaya TE, Gromov EV, Vitkovskaya NM, Schirmer J. Core-Level Electronic Spectra in $\operatorname{ADC}(2)$ Approximation for Polarization Propagator: Carbon Monoxide and Nitrogen Molecules. J Struct Chem. 2000;41:483-494.

[111] Butscher W, Kammer WE. Modification of Davidson's Method for the Calculation of Eigenvalues and Eigenvectors of Large Real-Symmetric Matrices: "Root Homing Procedure". J Comput Phys. 1976;20:313-325.

[112] Liang W, Fischer SA, Frisch MJ, Li X. Energy-Specific Linear Response TDHF/TDDFT for Calculating High-Energy Excited States. J Chem Theor Comput. 2011;7:3540-3547.

[113] Stratmann RE, Scuseria GE, Frisch MJ. An Efficient Implementation of Time-Dependent Density-Functional Theory for the Calculation of Excitation Energies of Large Molecules. J Chem Phys. 1998;109:8218-8224.

[114] Kasper JM, Williams-Young DB, Vecharynski E, Yang C, Li X. A Well-Tempered Hybrid Method for Solving Challenging Time-Dependent Density Functional Theory (TDDFT) Systems. J Chem Theor Comput. 2018;14:2034-2041.

[115] Buenker RJ, Peyerimhoff SD. All-Electron CI Calculations for Core-Ionized, Core-Valence Exclted and Shake-up States of $\mathrm{N}_{2}$. Chem Phys Lett. 1977;52:8.

[116] Lestrange PJ, Nguyen PD, Li X. Calibration of Energy-Specific TDDFT for Modeling K-Edge XAS Spectra of Light Elements. J Chem Theor Comput. 2015;11:2994-2999.

[117] Peng B, Lestrange PJ, Goings JJ, Caricato M, Li X. Energy-Specific Equation-of-Motion Coupled-Cluster Methods for High-Energy Excited States: Application to K-Edge X-Ray Absorption Spectroscopy. J Chem Theor Comput. 2015;11:4146-4153.

[118] Potts DM, Taylor CM, Chaudhuri RK, Freed KF. The Improved Virtual Orbital-Complete Active Space Configuration Interaction Method, a "Packageable" Efficient Ab Initio ManyBody Method for Describing Electronically Excited States. J Chem Phys. 2001;114:2592-2600.

[119] Thunemann KH, Römelt J, Peyerimhoff SD, Buenker RJ. A Study of the Convergence in Iterative Natural Orbital Procedures: Convergence in Two Procedures. Int J Quant Chem. 1977;11:743-752. 
[120] Hupp T, Engels B, Della Sala F, Görling A. Orbitals from a Self-Interaction Free Kohn-Sham Potential as a Single Electron Basis for Ab Initio Methods. Chem Phys Lett. 2002;360:175181.

[121] Barth A, Buenker RJ, Peyerimhoff SD, Butscher W. Theoretical Study of the Core-Ionized and Various Core-Excited and Shake-up States of Acetylene and Ethylene by Ab Initio MRDCI Methods. Chem Phys. 1980;46:149-164.

[122] Cambi R, Ciullo G, Sgamelotti A, Tarantelli F, Guest MF. A CI Investigation on the Core Ionized and Core-Rydberg Excited States of $\mathrm{NH}_{2}$. Chem Phys Lett. 1982;91:178-184.

[123] Marian CM, Heil A, Kleinschmidt M. The DFT/MRCI Method. Wiley Interdiscip Rev: Comput Mol Sci. 2018;p. e1394.

[124] Grimme S. Density Functional Calculations with Configuration Interaction for the Excited States of Molecules. Chem Phys Lett. 1996;259:128-137.

[125] Grimme S, Waletzke M. A Combination of Kohn-Sham Density Functional Theory and Multi-Reference Configuration Interaction Methods. J Chem Phys. 1999;111:5645-5655.

[126] Roemelt M, Maganas D, DeBeer S, Neese F. A Combined DFT and Restricted Open-Shell Configuration Interaction Method Including Spin-Orbit Coupling: Application to Transition Metal L-Edge X-Ray Absorption Spectroscopy. J Chem Phys. 2013;138:204101.

[127] Escudero D, Thiel W. Assessing the Density Functional Theory-Based Multireference Configuration Interaction (DFT/MRCI) Method for Transition Metal Complexes. J Chem Phys. 2014;140:194105.

[128] Ikeno H, Tanaka I, Koyama Y, Mizoguchi T, Ogasawara K. First-Principles Multielectron Calculations of $\mathrm{Ni} \mathrm{L}_{2,3}$ NEXAFS and ELNES for $\mathrm{LiNiO}_{2}$ and Related Compounds. Phys Rev B. 2005;72:075123.

[129] Ikeno H, Mizoguchi T, Koyama Y, Kumagai Y, Tanaka I. First-Principles Multi-Electron Calculations for $\mathrm{L}_{2,3}$ ELNES/XANES of 3d Transition Metal Monoxides. Ultramicroscopy. 2006;106:970-975. 
[130] Kumagai Y, Ikeno H, Oba F, Matsunaga K, Tanaka I. Effects of Crystal Structure on Co$\mathrm{L}_{2,3}$ x-Ray Absorption near-Edge Structure and Electron-Energy-Loss near-Edge Structure of Trivalent Cobalt Oxides. Phys Rev B. 2008;77:155124.

[131] Ikeno H. First-Principles Analysis of X-Ray Magnetic Circular Dichroism for Transition Metal Complex Oxides. J Appl Phys. 2016;120:142104.

[132] Ikeno H, Mizoguchi T, Tanaka I. Ab Initio Charge Transfer Multiplet Calculations on the $\mathrm{L}_{2,3}$ XANES and ELNES of 3d Transition Metal Oxides. Phys Rev B. 2011;83:155107.

[133] Kumagai Y, Ikeno H, Tanaka I. All-Electron CI Calculations of 3d Transition-Metal L $\mathrm{L}_{2,3}$ XANES Using Zeroth-Order Regular Approximation for Relativistic Effects. J Phys: Condens Matter. 2009;21:104209.

[134] Watanabe S, Kamimura H. First-Principles Calculations of Multiplet Structures of Transition Metal Deep Impurities in II-VI and III-V Semiconductors. Mater Sci Eng B. 1989;3:313-324.

[135] Ilton ES, Bagus PS. Ligand Field Effects on the Multiplet Structure of the U4f XPS of UO . $_{2}$ Surf Sci. 2008;602:1114-1121.

[136] Bagus PS, Ilton ES. Effects of Covalency on the P-Shell Photoemission of Transition Metals: MnO. Phys Rev B. 2006;73:155110.

[137] Maganas D, DeBeer S, Neese F. A Pair Natural Orbitals Restricted Open Shell Configuration Interaction (PNO-ROCIS) Approach for Calculating X-Ray Absorption Spectra of Large Chemical Systems. J Phys Chem A. 2018;

[138] Kubas A, Verkamp M, Vura-Weis J, Neese F, Maganas D. A Restricted Open Configuration Interaction Singles Study on M- and L-Edge X-Ray Absorption Spectroscopy of Solid Chemical Systems. J Chem Theor Comput. 2018;.

[139] Griffith JS, Orgel LE. Ligand-Field Theory. Q Rev Chem Soc. 1957;11:381.

[140] Cowan RD. The Theory of Atomic Structure and Spectra. Los Alamos Series in Basic and Applied Sciences. Berkeley: University of California Press; 1981.

[141] Thole BT, Cowan RD, Sawatzky GA, Fink J, Fuggle JC. New Probe for the Ground-State Electronic Structure of Narrow-Band and Impurity Systems. Phys Rev B. 1985;31:6856-6858. 
[142] Okada K, Kotani A, Thole BT. Charge Transfer Satellites and Multiplet Splitting in X-Ray Photoemission Spectra of Late Transition Metal Halides. J Electron Spectrosc Relat Phenom. 1992;58:325-343.

[143] Haverkort MW, Zwierzycki M, Andersen OK. Multiplet Ligand-Field Theory Using Wannier Orbitals. Phys Rev B. 2012;85:165113.

[144] Hariki A, Uozumi T, Kuneš J. LDA+DMFT Approach to Core-Level Spectroscopy: Application to 3d Transition Metal Compounds. Phys Rev B. 2017;96:045111.

[145] Wahl AC, Das G. The Multiconfiguration Self-Consistent Field Method. In: Schaefer HF, editor. Methods of Electronic Structure Theory. Boston, MA: Springer US; 1977. p. 51-78.

[146] McWeeny R, Sutcliffe BT. Fundamentals of Self-Consistent-Field (SCF), Hartree-Fock (HF), Multi-Configuration (MC)SCF and Configuration Interaction (CI) Schemes. Comput Phys Rep. 1985;2:218-278.

[147] Roos BO. Björn O. Roos Lecture Notes in Quantum Chemistry European Summer School in Quantum Chemistry. Lund: Springer-Verlag; 1992.

[148] Werner HJ. Matrix-Formulated Direct Multiconfiguration Self-Consistent Field and Multiconfiguration Reference Configuration-Interaction Methods. In: Lawley KP, editor. Adv. Chem. Phys. Hoboken, NJ, USA: John Wiley \& Sons, Inc.; 1987. p. 1-62.

[149] Shepard R. The Multiconfiguration Self-Consistent Field Method. In: Lawley KP, editor. Adv. Chem. Phys. Hoboken, NJ, USA: John Wiley \& Sons, Inc.; 1987. p. 63-200.

[150] Roos BO. The Complete Active Space Self-Consistent Field Method and Its Applications in Electronic Structure Calculations. In: Lawley KP, editor. Adv. Chem. Phys. Hoboken, NJ, USA: John Wiley \& Sons, Inc.; 1987. p. 399-445.

[151] Olsen J, Yeager DL, Jørgensen P. Optimization and Characterization of a Multiconfigurational Self-Consistent Field (MCSCF) State. In: Prigogine I, Rice SA, editors. Adv. Chem. Phys. Hoboken, NJ, USA: John Wiley \& Sons, Inc.; 2007. p. 1-176.

[152] Szalay PG, Müller T, Gidofalvi G, Lischka H, Shepard R. Multiconfiguration Self-Consistent Field and Multireference Configuration Interaction Methods and Applications. Chem Rev. 2012;112:108-181. 
[153] Lischka H, Nachtigallová D, Aquino AJA, Szalay PG, Plasser F, Machado FBC, et al. Multireference Approaches for Excited States of Molecules. Chem Rev. 2018;118:7293-7361.

[154] Schwarz WHE, Chang TC, Seeger U, Hwang KH. Core Excitations of Symmetrical Aromatic Molecules. Specific Correlations in the Valence Shell and Localization in the Core Shells. Chem Phys. 1987;117:73-89.

[155] Bacskay GB, Bryant G, Hush NS. Hole Localization and Broken Symmetry: A Theoretical Study of Core Electron Ionization in the $\mathrm{Li}_{2}$ Molecule. Int J Quant Chem. 1987;31:471-487.

[156] Josefsson I, Kunnus K, Schreck S, Föhlisch A, de Groot F, Wernet P, et al. Ab Initio Calculations of X-Ray Spectra: Atomic Multiplet and Molecular Orbital Effects in a Multiconfigurational SCF Approach to the L-Edge Spectra of Transition Metal Complexes. J Phys Chem Lett. 2012;3:3565-3570.

[157] Bokarev SI, Dantz M, Suljoti E, Kühn O, Aziz EF. State-Dependent Electron Delocalization Dynamics at the Solute-Solvent Interface: Soft-X-Ray Absorption Spectroscopy and Ab Initio Calculations. Phys Rev Lett. 2013;111:083002.

[158] Pinjari RV, Delcey MG, Guo M, Odelius M, Lundberg M. Restricted Active Space Calculations of L-Edge X-Ray Absorption Spectra: From Molecular Orbitals to Multiplet States. J Chem Phys. 2014;141:124116.

[159] Engels B, Hanrath M, Lennartz C. Individually Selecting Multi-Reference CI and Its Application to Biradicalic Cyclizations. Comput Chem. 2001;25:15-38.

[160] Bytautas L, Ruedenberg K. A Priori Identification of Configurational Deadwood. Chem Phys. 2009;356:64-75.

[161] Roos BO, Taylor PR, Siegbahn PEM. A Complete Active Space SCF Method (CASSCF) Using a Density Matrix Formulated Super-CI Approach. Chem Phys. 1980;48:157-173.

[162] Olsen J, Roos BO, Jørgensen P, Jensen HJA. Determinant Based Configuration Interaction Algorithms for Complete and Restricted Configuration Interaction Spaces. J Chem Phys. 1988;89:2185. 
[163] Malmqvist PA, Rendell A, Roos BO. The Restricted Active Space Self-Consistent-Field Method, Implemented with a Split Graph Unitary Group Approach. J Phys Chem. 1990;94:5477-5482.

[164] Fleig T, Olsen J, Marian CM. The Generalized Active Space Concept for the Relativistic Treatment of Electron Correlation. I. Kramers-Restricted Two-Component Configuration Interaction. J Chem Phys. 2001;114:4775-4790.

[165] Ma D, Li Manni G, Gagliardi L. The Generalized Active Space Concept in Multiconfigurational Self-Consistent Field Methods. J Chem Phys. 2011;135:044128.

[166] Ivanic J. Direct Configuration Interaction and Multiconfigurational Self-Consistent-Field Method for Multiple Active Spaces with Variable Occupations. I. Method. J Chem Phys. 2003;119:9364-9376.

[167] Roos BO, Andersson K, Fülscher MP, Malmqvist Pt, Serrano-Andrés L, Pierloot K, et al. Multiconfigurational Perturbation Theory: Applications in Electronic Spectroscopy. In: Prigogine I, Rice SA, editors. Adv. Chem. Phys.. vol. 93. 1st ed. New York: John Wiley \& Sons, Inc.; 1996. p. 219-332.

[168] Pierloot K. Nondynamic Correlation Effects in Transition Metal Coordination Compounds. In: Cundari TR, editor. Computational Organometallic Chemistry. New York: Marcel Dekker; 2001. p. 123-158.

[169] Pierloot K. The CASPT2 Method in Inorganic Electronic Spectroscopy: From Ionic Transition Metal to Covalent Actinide Complexes. Mol Phys. 2003;101:2083-2094.

[170] Veryazov V, Malmqvist PA, Roos BO. How to Select Active Space for Multiconfigurational Quantum Chemistry? Int J Quant Chem. 2011;111:3329-3338.

[171] Atak K, Bokarev SI, Gotz M, Golnak R, Lange KM, Engel N, et al. Nature of the Chemical Bond of Aqueous $\mathrm{Fe}^{2+}$ Probed by Soft X-Ray Spectroscopies and Ab Initio Calculations. J Phys Chem B. 2013;117:12613-12618.

[172] Chantzis A, Kowalska JK, Maganas D, DeBeer S, Neese F. Ab Initio Wavefunction-Based Determination of Element Specific Shifts for the Efficient Calculation of X-Ray Absorption Spectra of Main Group Elements and First Row Transition Metals. J Chem Theor Comput. 2018 ;. 
[173] Firsht D, McWeeny R. SCF Theory for Excited States: II. Some Applications to Hole States of Free Atoms. Mol Phys. 1976;32:1637-1649.

[174] Pinjari RV, Delcey MG, Guo M, Odelius M, Lundberg M. Cost and Sensitivity of Restricted Active-Space Calculations of Metal L-Edge X-Ray Absorption Spectra: Effects of the ActiveSpace Selection on the L-Edge XAS Spectra. J Comput Chem. 2016;37:477-486.

[175] Norell J, Jay R, Hantschmann M, Eckert S, Guo M, Gaffney K, et al. Fingerprints of Electronic, Spin and Structural Dynamics from Resonant Inelastic Soft x-Ray Scattering in Transient Photo-Chemical Species. Phys Chem Chem Phys. 2018;20:7243.

[176] Bokarev, SI, Hilal, R, Aziz, SG, Kühn, O, Soft X-ray spectroscopy of transition metal compounds: a theoretical perspective. EPJ Web of Conf. 2017;132,02004.

[177] Werner HJ, Meyer W. A Quadratically Convergent MCSCF Method for the Simultaneous Optimization of Several States. J Chem Phys. 1981;74:5794-5801.

[178] Gross EKU, Oliveira LN, Kohn W. Rayleigh-Ritz Variational Principle for Ensembles of Fractionally Occupied States. Phys Rev A. 1988;37:2805-2808.

[179] McWeeny R. SCF Theory for Excited States: I. Optimal Orbitals for the States of a Configuration. Mol Phys. 1974;28:1273-1282.

[180] Sergentu DC, Duignan TJ, Autschbach J. Ab Initio Study of Covalency in the Ground versus Core-Excited States and X-Ray Absorption Spectra of Actinide Complexes. J Phys Chem Lett. 2018;9:5583-5591.

[181] Hirao K. Multireference Møller-Plesset Method. Chem Phys Lett. 1992;190:374-380.

[182] Hirao K. Multireference Møller-Plesset Perturbation Theory for High-Spin Open-Shell Systems. Chem Phys Lett. 1992;196:397-403.

[183] Angeli C, Cimiraglia R, Evangelisti S, Leininger T, Malrieu JP. Introduction of $N$-Electron Valence States for Multireference Perturbation Theory. J Chem Phys. 2001;114:10252-10264.

[184] Angeli C, Cimiraglia R, Malrieu JP. N-Electron Valence State Perturbation Theory: A Fast Implementation of the Strongly Contracted Variant. Chem Phys Lett. 2001;350:297-305. 
[185] Granovsky AA. Extended Multi-Configuration Quasi-Degenerate Perturbation Theory: The New Approach to Multi-State Multi-Reference Perturbation Theory. J Chem Phys. 2011;134:214113.

[186] Andersson K, Malmqvist PA, Roos BO, Sadlej AJ, Wolinski K. Second-Order Perturbation Theory with a CASSCF Reference Function. J Phys Chem. 1990;94:5483-5488.

[187] Andersson K, Malmqvist PA, Roos BO. Second-order Perturbation Theory with a Complete Active Space Self-consistent Field Reference Function. J Chem Phys. 1992;96:1218-1226.

[188] Roos BO, Fülscher M, Malmqvist PA, Merchán M, Serrano-Andrés L. Theoretical Studies of the Electronic Spectra of Organic Molecules. In: Langhoff SR, editor. Quantum Mechanical Electronic Structure Calculations with Chemical Accuracy. Dordrecht: Springer Netherlands; 1995. p. $357-438$.

[189] Pulay P. A Perspective on the CASPT2 Method. Int J Quant Chem. 2011;111:3273-3279.

[190] Finley J, Malmqvist PA, Roos BO, Serrano-Andrés L. The Multi-State CASPT2 Method. Chem Phys Lett. 1998;288:299-306.

[191] Roos BO, Andersson K. Multiconfigurational Perturbation Theory with Level Shift — the $\mathrm{Cr}_{2}$ Potential Revisited. Chem Phys Lett. 1995;245:215-223.

[192] Roos BO, Andersson K, Fülscher MP, Serrano-Andrés L, Pierloot K, Merchán M, et al. Applications of Level Shift Corrected Perturbation Theory in Electronic Spectroscopy. J Mol Struct THEOCHEM. 1996;388:257-276.

[193] Forsberg N, Malmqvist PA. Multiconfiguration Perturbation Theory with Imaginary Level Shift. Chem Phys Lett. 1997;274:196-204.

[194] Kubin M, Guo M, Ekimova M, Källman E, Kern J, Yachandra VK, et al. Cr L-Edge X-Ray Absorption Spectroscopy of $\mathrm{Cr}^{\mathrm{III}}$ (Acac) ${ }_{3}$ in Solution with Measured and Calculated Absolute Absorption Cross Sections. J Phys Chem B. 2018;122:7375-7384.

[195] Schröter, M, Ivanov, SD, Schulze, J, Polyutov, SP, Yan, Y, Pullerits, T, Kühn, O ExcitonVibrational Coupling in the Dynamics and Spectroscopy of Frenkel Excitons in Molecular Aggregates. Phys Rep. 2015;567,1-78. 
[196] Preuße M, Bokarev SI, Aziz SG, Kühn O. Towards an Ab Initio Theory for Metal L-Edge Soft X-Ray Spectroscopy of Molecular Aggregates. Struct Dyn. 2016;3:062601.

[197] Kunnus K, Josefsson I, Rajkovic I, Schreck S, Quevedo W, Beye M, et al. Identification of the Dominant Photochemical Pathways and Mechanistic Insights to the Ultrafast Ligand Exchange of $\mathrm{Fe}(\mathrm{CO})_{5}$ to $\mathrm{Fe}(\mathrm{CO})_{4}$ EtOH. Struct Dyn. 2016;3:043204.

[198] Klooster R, Broer R, Filatov M. Calculation of X-Ray Photoelectron Spectra with the Use of the Normalized Elimination of the Small Component Method. Chem Phys. 2012;395:122-127.

[199] Grell G, Bokarev SI, Winter B, Seidel R, Aziz EF, Aziz SG, et al. Erratum: "Multi-Reference Approach to the Calculation of Photoelectron Spectra Including Spin-Orbit Coupling" [J. Chem. Phys. 143 , 074104 (2015)]. J Chem Phys. 2016;145:089901.

[200] Wang H, Bokarev SI, Aziz SG, Kühn O. Ultrafast Spin-State Dynamics in Transition-Metal Complexes Triggered by Soft-X-Ray Light. Phys Rev Lett. 2017;118:023001.

[201] Goings JJ, Lestrange PJ, Li X. Real-Time Time-Dependent Electronic Structure Theory. Wiley Interdiscip Rev: Comput Mol Sci. 2018;8:e1341.

[202] Sato T, Ishikawa KL. Time-Dependent Multiconfiguration Self-Consistent-Field Method Based on the Occupation-Restricted Multiple-Active-Space Model for Multielectron Dynamics in Intense Laser Fields. Phys Rev A. 2015;91:023417.

[203] Meyer HD. Studying Molecular Quantum Dynamics with the Multiconfiguration TimeDependent Hartree Method: Multiconfiguration Time-Dependent Hartree. Wiley Interdiscip Rev: Comput Mol Sci. 2012;2:351-374.

[204] Lopata K, Van Kuiken BE, Khalil M, Govind N. Linear-Response and Real-Time TimeDependent Density Functional Theory Studies of Core-Level Near-Edge X-Ray Absorption. J Chem Theor Comput. 2012;8:3284-3292.

[205] Kadek M, Konecny L, Gao B, Repisky M, Ruud K. X-Ray Absorption Resonances near L $2_{2,3}^{-}$ Edges from Real-Time Propagation of the Dirac-Kohn-Sham Density Matrix. Phys Chem Chem Phys. 2015;17:22566-22570. 
[206] Kasper JM, Lestrange PJ, Stetina TF, Li X. Modeling L L,3-Edge X-Ray Absorption Spectroscopy with Real-Time Exact Two-Component Relativistic Time-Dependent Density Functional Theory. J Chem Theor Comput. 2018;14:1998-2006.

[207] Mendive-Tapia D, Vacher M, Bearpark M, Robb MA. Coupled Electron-Nuclear Dynamics: Charge Migration and Charge Transfer Initiated near a Conical Intersection. J Chem Phys. 2013;139:044110.

[208] Bauch S, Sørensen LK, Madsen LB. Time-Dependent Generalized-Active-Space Configuration-Interaction Approach to Photoionization Dynamics of Atoms and Molecules. Phys Rev A. 2014;90:062508.

[209] Li Z, Vendrell O, Santra R. Ultrafast Charge Transfer of a Valence Double Hole in Glycine Driven Exclusively by Nuclear Motion. Phys Rev Lett. 2015;115:143002.

[210] Kuleff AI, Lorenz S Cederbaum. Radiation Generated by the Ultrafast Migration of a Positive Charge Following the Ionization of a Molecular System. Phys Rev Lett. 2011;106:053001.

[211] Nascimento DR, DePrince AE. Simulation of Near-Edge X-Ray Absorption Fine Structure with Time-Dependent Equation-of-Motion Coupled-Cluster Theory. J Phys Chem Lett. $2017 ; 8: 2951-2957$.

[212] Wang H, Bokarev SI, Aziz SG, Kühn O. Density Matrix-Based Time-Dependent Configuration Interaction Approach to Ultrafast Spin-Flip Dynamics. Mol Phys. 2017;115:1898-1907.

[213] Wang H, Möhle T, Kühn O, Bokarev SI. Ultrafast Dissipative Spin-State Dynamics Triggered by x-Ray Pulse Trains. Phys Rev A. 2018;98:013408.

[214] Kubo R. Statistical-Mechanical Theory of Irreversible Processes. I. General Theory and Simple Applications to Magnetic and Conduction Problems. J Phys Soc Jpn. 1957;12:570586.

[215] Oddershede J. Propagator Methods. In: Lawley KP, editor. Adv. Chem. Phys. Hoboken, NJ, USA: John Wiley \& Sons, Inc.; 1987. p. 201-239.

[216] Cederbaum LS, Domcke W. Theoretical Aspects of Ionization Potentials and Photoelectron Spectroscopy: A Green's Function Approach. In: Prigogine I, Rice SA, editors. Adv. Chem. Phys. New York; London: John Wiley; 1977. p. 205-344. 
[217] Schirmer J. Beyond the Random-Phase Approximation: A New Approximation Scheme for the Polarization Propagator. Phys Rev A. 1982;26:2395-2416.

[218] Schirmer J. Theoretical Study of K-Shell Excitations in Formaldehyde. Chem Phys. $1988 ; 122: 9-15$.

[219] Wenzel J, Wormit M, Dreuw A. Calculating Core-Level Excitations and x-Ray Absorption Spectra of Medium-Sized Closed-Shell Molecules with the Algebraic-Diagrammatic Construction Scheme for the Polarization Propagator. J Comput Chem. 2014;35:1900-1915.

[220] Wenzel J, Holzer A, Wormit M, Dreuw A. Analysis and Comparison of CVS-ADC Approaches up to Third Order for the Calculation of Core-Excited States. J Chem Phys. 2015;142:214104.

[221] Rehn DR, Dreuw A, Norman P. Resonant Inelastic X-Ray Scattering Amplitudes and Cross Sections in the Algebraic Diagrammatic Construction/Intermediate State Representation (ADC/ISR) Approach. J Chem Theor Comput. 2017;13:5552-5559.

[222] Pernpointner M, Rapps T, Cederbaum LS. Jahn-Teller Distortions in the Photodetachment Spectrum of $\mathrm{PtCl}_{6}^{2-}$ : A Four-Component Relativistic Study. J Chem Phys. 2009;131:044322.

[223] Kryzhevoi NV, Cederbaum LS. Core Ionization of $\mathrm{Na}^{+}$microsolvated in Water and Ammonia. J Chem Phys. 2009;130:084302.

[224] Neville SP, Averbukh V, Ruberti M, Yun R, Patchkovskii S, Chergui M, et al. Excited State X-Ray Absorption Spectroscopy: Probing Both Electronic and Structural Dynamics. J Chem Phys. 2016;145:144307.

[225] Gokhberg K, Averbukh V, Cederbaum LS. Decay Rates of Inner-Valence Excitations in Noble Gas Atoms. J Chem Phys. 2007;126:154107.

[226] Kuleff AI, Cederbaum LS. Tracing Ultrafast Interatomic Electronic Decay Processes in Real Time and Space. Phys Rev Lett. 2007;98:083201.

[227] Dreuw A, Head-Gordon M. Single-Reference Ab Initio Methods for the Calculation of Excited States of Large Molecules. Chem Rev. 2005;105:4009-4037.

[228] Peach MJG, Benfield P, Helgaker T, Tozer DJ. Excitation Energies in Density Functional Theory: An Evaluation and a Diagnostic Test. J Chem Phys. 2008;128:44118. 
[229] Besley NA, Peach MJG, Tozer DJ. Time-Dependent Density Functional Theory Calculations of near-Edge X-Ray Absorption Fine Structure with Short-Range Corrected Functionals. Phys Chem Chem Phys. 2009;11:10350.

[230] Besley NA, Noble A. Time-Dependent Density Functional Theory Study of the X-Ray Absorption Spectroscopy of Acetylene, Ethylene, and Benzene on Si(100). J Phys Chem C. 2007;111:3333-3340.

[231] Bokarev SI, Bokareva OS, Kühn O. Electronic Excitation Spectrum of the Photosensitizer $\left[\operatorname{Ir}(\text { Ppy })_{2}(\text { Bpy })\right]^{+}$. J Chem Phys. 2012;136:214305.

[232] Baer R, Livshits E, Salzner U. Tuned Range-Separated Hybrids in Density Functional Theory. Annu Rev Phys Chem. 2010;61:85-109.

[233] Nakata A, Imamura Y, Otsuka T, Nakai H. Time-Dependent Density Functional Theory Calculations for Core-Excited States: Assessment of Standard Exchange-Correlation Functionals and Development of a Novel Hybrid Functional. J Chem Phys. 2006;124:094105.

[234] Song JW, Watson MA, Nakata A, Hirao K. Core-Excitation Energy Calculations with a LongRange Corrected Hybrid Exchange-Correlation Functional Including a Short-Range Gaussian Attenuation (LCgau-BOP). J Chem Phys. 2008;129:184113.

[235] Imamura Y, Nakai H. Analysis of Self-Interaction Correction for Describing Core Excited States. Int J Quant Chem. 2007;107:23-29.

[236] Tu G, Rinkevicius Z, Vahtras O, Ågren H, Ekström U, Norman P, et al. Self-InteractionCorrected Time-Dependent Density-Functional-Theory Calculations of x-Ray-Absorption Spectra. Phys Rev A. 2007;76:022506.

[237] Verma P, Bartlett RJ. Increasing the Applicability of Density Functional Theory. V. X-Ray Absorption Spectra with Ionization Potential Corrected Exchange and Correlation Potentials. J Chem Phys. 2016;145:034108.

[238] Baseggio O, Toffoli D, Stener M, Fronzoni G, de Simone M, Grazioli C, et al. S2p Core Level Spectroscopy of Short Chain Oligothiophenes. J Chem Phys. 2017;147:244301.

[239] Campbell L, Mukamel S. Simulation of X-Ray Absorption near Edge Spectra of Electronically Excited Ruthenium Tris-2,2'-Bipyridine. J Chem Phys. 2004;121:12323. 
[240] Mukamel S, Healion D, Zhang Y, Biggs JD. Multidimensional Attosecond Resonant XRay Spectroscopy of Molecules: Lessons from the Optical Regime. Annu Rev Phys Chem. 2013;64:101-127.

[241] Zhang Y, Hua W, Bennett K, Mukamel S. Nonlinear Spectroscopy of Core and Valence Excitations Using Short X-Ray Pulses: Simulation Challenges. Top Curr Chem. 2015;368:273-345.

[242] Bartlett RJ, MusiałM. Coupled-Cluster Theory in Quantum Chemistry. Rev Mod Phys. 2007;79:291-352.

[243] Bartlett RJ. Coupled-Cluster Theory and Its Equation-of-Motion Extensions: CoupledCluster Theory. Wiley Interdiscip Rev: Comput Mol Sci. 2012;2:126-138.

[244] Koch H, Jørgensen P. Coupled Cluster Response Functions. J Chem Phys. 1990;93:3333-3344.

[245] Koch H, Kobayashi R, Sanchez de Merás A, Jørgensen P. Calculation of Size-intensive Transition Moments from the Coupled Cluster Singles and Doubles Linear Response Function. J Chem Phys. 1994;100:4393-4400.

[246] Besley NA. Equation of Motion Coupled Cluster Theory Calculations of the X-Ray Emission Spectroscopy of Water. Chem Phys Lett. 2012;542:42-46.

[247] Bazante AP, Perera A, Bartlett RJ. Towards Core-Excitation Spectra in Attosecond Spectroscopy: A Coupled-Cluster Study of ClF. Chem Phys Lett. 2017;683:68-75.

[248] Nooijen M, Bartlett RJ. Description of Core-excitation Spectra by the Open-shell Electronattachment Equation-of-motion Coupled Cluster Method. J Chem Phys. 1995;102:6735-6756.

[249] Coriani S, Koch H. Communication: X-Ray Absorption Spectra and Core-Ionization Potentials within a Core-Valence Separated Coupled Cluster Framework. J Chem Phys. 2015;143:181103.

[250] Myhre RH, Coriani S, Koch H. Near-Edge X-Ray Absorption Fine Structure within Multilevel Coupled Cluster Theory. J Chem Theor Comput. 2016;12:2633-2643.

[251] Lyakh DI, MusiałM, Lotrich VF, Bartlett RJ. Multireference Nature of Chemistry: The Coupled-Cluster View. Chem Rev. 2012;112:182-243. 
[252] Brabec J, Bhaskaran-Nair K, Govind N, Pittner J, Kowalski K. Communication: Application of State-Specific Multireference Coupled Cluster Methods to Core-Level Excitations. J Chem Phys. 2012;137:171101.

[253] Dutta AK, Gupta J, Vaval N, Pal S. Intermediate Hamiltonian Fock Space Multireference Coupled Cluster Approach to Core Excitation Spectra. J Chem Theor Comput. 2014;10:36563668 .

[254] Sen S, Shee A, Mukherjee D. Inclusion of Orbital Relaxation and Correlation through the Unitary Group Adapted Open Shell Coupled Cluster Theory Using Non-Relativistic and Scalar Relativistic Hamiltonians to Study the Core Ionization Potential of Molecules Containing Light to Medium-Heavy Elements. J Chem Phys. 2018;148:054107.

[255] Pitzer KS. Relativistic Effects on Chemical Properties. Acc Chem Res. 1979;12:271-276.

[256] Pyykko P, Desclaux JP. Relativity and the Periodic System of Elements. Acc Chem Res. 1979;12:276-281.

[257] Rose SJ, Grant IP, Pyper NC. The Direct and Indirect Effects in the Relativistic Modification of Atomic Valence Orbitals. J Phys B. 1978;11:1171-1176.

[258] Wilson S. Methods in Computational Chemistry: Volume 2 Relativistic Effects in Atoms and Molecules; 1988.

[259] Schwerdtfeger P, editor. Relativistic Electronic Structure Theory. 1st ed. Theoretical and Computational Chemistry. Amsterdam: Elsevier Science; 2002.

[260] Hess BA, editor. Relativistic Effects in Heavy-Element Chemistry and Physics. Wiley Series in Theoretical Chemistry. Chichester, West Sussex, England ; Hoboken NJ: J. Wiley; 2003.

[261] Grant IP. Relativistic Quantum Theory of Atoms and Molecules Theory and Computation. New York: Springer; 2007.

[262] Dyall KG, Fægri K. Introduction to Relativistic Quantum Chemistry. New York: Oxford University Press; 2007.

[263] Reiher M, Wolf A. Relativistic Quantum Chemistry. Weinheim: Wiley-VCH; 2009. 
[264] Ekström U, Norman P, Carravetta V. Relativistic Four-Component Static-Exchange Approximation for Core-Excitation Processes in Molecules. Phys Rev A. 2006;73:022501.

[265] Fransson T, Burdakova D, Norman P. K- and L-Edge X-Ray Absorption Spectrum Calculations of Closed-Shell Carbon, Silicon, Germanium, and Sulfur Compounds Using Damped Four-Component Density Functional Response Theory. Phys Chem Chem Phys. 2016;18:13591-13603.

[266] Pernpointner M, Cederbaum LS. Effect of Relativity on the Ionization Spectra of the Xenon Fluorides $\mathrm{XeF}_{n}(\mathrm{~N}=2,4,6)$. J Chem Phys. 2005;122:214302.

[267] Pernpointner M, Knecht S, Cederbaum LS. Ionization Spectra and Electronic Decay in Small Iodide Clusters: Fully Relativistic Results. J Chem Phys. 2006;125:034309.

[268] Peng D, Reiher M. Exact Decoupling of the Relativistic Fock Operator. Theor Chem Acc. $2012 ; 131$.

[269] Chang C, Pelissier M, Durand P. Regular Two-Component Pauli-Like Effective Hamiltonians in Dirac Theory. Phys Scr. 1986;34:394-404.

[270] Barysz M, Sadlej AJ. Infinite-Order Two-Component Theory for Relativistic Quantum Chemistry. J Chem Phys. 2002;116:2696-2704.

[271] Hess BA. Applicability of the No-Pair Equation with Free-Particle Projection Operators to Atomic and Molecular Structure Calculations. Phys Rev A. 1985;32:756-763.

[272] Hess BA. Relativistic Electronic-Structure Calculations Employing a Two-Component NoPair Formalism with External-Field Projection Operators. Phys Rev A. 1986;33:3742.

[273] Casarin M, Finetti P, Vittadini A, Wang F, Ziegler T. Spin-Orbit Relativistic TimeDependent Density Functional Calculations of the Metal and Ligand Pre-Edge XAS Intensities of Organotitanium Complexes: $\mathrm{TiCl}_{4}, \mathrm{Ti}\left(\eta_{5}-\mathrm{C}_{5} \mathrm{H}_{5}\right) \mathrm{Cl}_{3}$, and $\mathrm{Ti}\left(\eta_{5}-\mathrm{C}_{5} \mathrm{H}_{5}\right)_{2} \mathrm{Cl}_{2}$. J Phys Chem A. 2007;111:5270-5279.

[274] Fronzoni G, Stener M, Decleva P, Wang F, Ziegler T, van Lenthe E, et al. Spin-Orbit Relativistic Time Dependent Density Functional Theory Calculations for the Description of Core Electron Excitations: $\mathrm{TiCl}_{4}$ Case Study. Chem Phys Lett. 2005;416:56-63. 
[275] Shakhova VM, Semenov SG, Lomachuk YV, Demidov YA, Skripnikov LV, Mosyagin NS, et al. Chemical Shift of the $\mathrm{K}_{\alpha 1}$ and $\mathrm{K}_{\alpha 2}$ Lines of the X-Ray Emission Spectrum of $\mathrm{Yb}(\mathrm{II}) / \mathrm{Yb}(\mathrm{III})$ Fluorides: A Quantum-Chemical Investigation. Opt Spectrosc. 2018;124:462-467.

[276] Lomachuk YV, Demidov YA, Skripnikov LV, Zaitsevskii AV, Semenov SG, Mosyagin NS, et al. Calculation of Chemical Shifts of X-Ray-Emission Spectra of Niobium in Niobium(V) Oxides Relative to Metal. Opt Spectrosc. 2018;124:472-477.

[277] Fronzoni G, Stener M, Decleva P, de Simone M, Coreno M, Franceschi P, et al. X-Ray Absorption Spectroscopy of $\mathrm{VOCl}_{3}, \mathrm{CrO}_{2} \mathrm{Cl}_{2}$, and $\mathrm{MnO}_{3} \mathrm{Cl}$ : An Experimental and Theoretical Study. J Phys Chem A. 2009;113:2914-2925.

[278] Barysz M, Syrocki 1. Relativistic Calculations of X-Ray Photoelectron Spectra and the Accuracy of the IOTC Method. Mol Phys. 2014;112:583-591.

[279] Verma P, Derricotte WD, Evangelista FA. Predicting Near Edge X-Ray Absorption Spectra with the Spin-Free Exact-Two-Component Hamiltonian and Orthogonality Constrained Density Functional Theory. J Chem Theor Comput. 2016;12:144-156.

[280] Marian CM. Spin-Orbit Coupling in Molecules. In: Lipkowitz KB, Boyd DB, John Wiley \& Sons, editors. Rev. Comput. Chem.. vol. 17. New York: Wiley-VCH; 2001. p. 99-204.

[281] Bethe HA, Salpeter EE. Quantum Mechanics of One- and Two-Electron Atoms. Boston, MA: Springer US; 1977.

[282] HeßBA, Marian CM, Wahlgren U, Gropen O. A Mean-Field Spin-Orbit Method Applicable to Correlated Wavefunctions. Chem Phys Lett. 1996;251:365-371.

[283] Schimmelpfennig B. AMFI, An Atomic Mean-Field Spin-Orbit Integral Program; 1996.

[284] Dolg M, Cao X. Relativistic Pseudopotentials: Their Development and Scope of Applications. Chem Rev. 2012;112:403-480.

[285] Balabanov NB, Peterson KA. Systematically Convergent Basis Sets for Transition Metals. I. All-Electron Correlation Consistent Basis Sets for the 3d Elements Sc-Zn. J Chem Phys. 2005;123:064107.

[286] Roos BO, Lindh R, Malmqvist PA, Veryazov V, Widmark PO. New Relativistic ANO Basis Sets for Transition Metal Atoms. J Phys Chem A. 2005;109:6575-6579. 
[287] Kutzelnigg W, Fleischer U, Schindler M. The IGLO-Method: Ab-Initio Calculation and Interpretation of NMR Chemical Shifts and Magnetic Susceptibilities. In: Diehl P, Fluck E, Günther H, Kosfeld R, Seelig J, editors. Deuterium and Shift Calculation. vol. 23. Berlin, Heidelberg: Springer Berlin Heidelberg; 1990. p. 165-262.

[288] Hahn AW, Van Kuiken BE, Chilkuri VG, Levin N, Bill E, Weyhermüller T, et al. Probing the Valence Electronic Structure of Low-Spin Ferrous and Ferric Complexes Using 2p3d Resonant Inelastic X-Ray Scattering (RIXS). Inorg Chem. 2018;57:9515-9530.

[289] de Groot FMF, Arrio MA, Sainctavit P, Cartier C, Chen CT. Fluorescence Yield Detection: Why It Does Not Measure the X-Ray Absorption Cross Section. Solid State Commun. 1994;92:991-995.

[290] Kurian R, Kunnus K, Wernet P, Butorin SM, Glatzel P, de Groot FMF. Intrinsic Deviations in Fluorescence Yield Detected X-Ray Absorption Spectroscopy: The Case of the Transition Metal L 2,3 Edges. J Phys: Condens Matter. 2012;24:452201.

[291] Wiechen M, Berends HM, Kurz P. Wateroxidation Catalysed by Manganese Compounds: From Complexes to 'Biomimetic Rocks'. Dalton Trans. 2012;41:21-31.

[292] Hocking RK, Brimblecombe R, Chang LY, Singh A, Cheah MH, Glover C, et al. WaterOxidation Catalysis by Manganese in a Geochemical-like Cycle. Nat Chem. 2011;3:461-466.

[293] Singh A, Hocking RK, Chang SLY, George BM, Fehr M, Lips K, et al. Water Oxidation Catalysis by Nanoparticulate Manganese Oxide Thin Films: Probing the Effect of the Manganese Precursors. Chem Mater. 2013;25:1098-1108.

[294] Khan M, Suljoti E, Singh A, Bonke SA, Brandenburg T, Atak K, et al. Electronic Structural Insights into Efficient $\mathrm{MnO}_{x}$ Catalysts. J Mater Chem A. 2014;2:18199-18203.

[295] Xiao J, Khan M, Singh A, Suljoti E, Spiccia L, Aziz EF. Enhancing Catalytic Activity by Narrowing Local Energy Gaps-X-Ray Studies of a Manganese Water Oxidation Catalyst. ChemSusChem. 2015;8:872-877.

[296] Atak K, Golnak R, Xiao J, Suljoti E, Pflüger M, Brandenburg T, et al. Electronic Structure of Hemin in Solution Studied by Resonant X-Ray Emission Spectroscopy and Electronic Structure Calculations. J Phys Chem B. 2014;118:9938-9943. 
[297] Golnak R, Xiao J, Atak K, Khan M, Suljoti E, Aziz EF. Local Energy Gap Opening Induced by Hemin Dimerization in Aqueous Solution. J Phys Chem B. 2015;119:3058-3062.

[298] Stein CJ, Reiher M. Automated Selection of Active Orbital Spaces. J Chem Theor Comput. $2016 ; 12: 1760-1771$. 